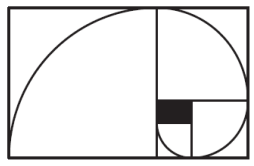

\title{
Algebraic structures related to integrable differential equations
}

\section{Vladimir Sokolov}

\begin{abstract}
This survey is devoted to algebraic structures related to integrable ODEs and evolution PDEs. A description of Lax representations is given in terms of vector space decomposition of loop algebras into direct sum of Taylor series and a complementary subalgebras. Examples of complementary subalgebras and corresponding integrable models are presented. In the frame of the bi-Hamiltonian approach, compatible associative algebras related affine Dynkin diagrams are considered. A bi-Hamiltonian origin of the classical elliptic Calogero-Moser models is revealed.
\end{abstract}





\section{Contents}

1 Introduction 5

1.1 List of basic notation . . . . . . . . . . . . . . 6

1.1.1 Constants, vectors and matrices . . . . . . . 6

1.1.2 Derivations and differential operators . . . . . . 6

1.1.3 Differential algebra . . . . . . . . . . . . . . 6

1.1 .4 Algebra . . . . . . . . . . . . . . 7

1.2 Lax pairs . . . . . . . . . . . . . . . . . 7

1.3 Hamiltonian structures . . . . . . . . . . . . . . . . 10

1.4 Bi-Hamiltonian formalism . . . . . . . . . . . . . . 12

1.4.1 Shift argument method . . . . . . . . . . . 13

1.4.2 Bi-Hamiltonian form for KdV equation . . . . . . 13

2 Factorization of Lie algebras and Lax pairs 15

2.1 Scalar Lax pairs for evolution equations . . . . . . . . . 15

2.1.1 Pseudo-differential series . . . . . . . . . . . . . . 17

2.1.2 Korteweg-de Vries hierarchy . . . . . . . . . . . . 19

2.1.3 Gelfand-Dikii hierarchy and generalizations . . . . . 26

2.2 Matrix Lax pairs . . . . . . . . . . . . . . . . . . 30

2.2 .1 The NLS hierarchy . . . . . . . . . . . . . . 30

2.2 .2 Generalizations . . . . . . . . . . . . . 33

2.3 Decomposition of loop algeras and Lax pairs . . . . . . . . 36

2.3.1 Factoring subalgebras for $\mathcal{G}=\mathfrak{s o}_{3} \ldots \ldots \ldots$. . . 40

2.3.2 Integrable top-like systems . . . . . . . . . . . . 44

$2.3 .3 \quad \mathfrak{s o}_{3}$ classical spinning tops . . . . . . . . . . . . 45

2.3.4 Generalization of Euler and Steklov-Lyapunov cases to the $\mathfrak{s o}_{n}$-case . . . . . . . . . . . 47

2.3.5 Factoring subalgebras for Kac-Moody algebras . . . 48

2.3.6 Integrable PDEs of the Landau-Lifshitz type . . . . 50

2.3.7 Hyperbolic models of chiral type . . . . . . . . . 52

2.4 Factorization method and non-associative algebras . . . . . 53

2.4.1 Factorization method . . . . . . . . . . . . 54

2.4 .2 Reductions . . . . . . . . . . . . . . 56 
2.4.3 Generalized factorization method . . . . . . . . 58

3 Algebraic structures in bi-Hamiltonian approach $\quad 61$

3.1 Polynomial forms for elliptic Calogero-Moser systems . . . . 61

3.1.1 Calogero-Moser Hamiltonians . . . . . . . . . . . . 61

3.1.2 Quasi-solvable differential operators . . . . . . . 63

3.1.3 Commutative subalgebras in $U\left(\mathfrak{g l}_{N+1}\right)$ and quantum

Calogero-Moser Hamiltonians . . . . . . . . . . 67

3.1.4 Bi-Hamiltonian origin of classical elliptic Calogero-

Moser models . . . . . . . . . . . . . . . 70

3.2 Bi-Hamiltonian formalism and compatible algebras . . . . . 74

3.2.1 Compatible Lie algebras . . . . . . . . . . . . . 74

3.2.2 Compatible associative algebras . . . . . . . . . 79

3.3 Non-abelian Hamiltonian formalism and trace Poisson

brackets ...................... 91

3.3.1 Non-abelian Poisson brackets on free associative algebras . . . . . . . . . . . . . . . 9 91

3.3.2 Trace Poisson brackets . . . . . . . . . . . . . . 95

3.3.3 Double Poisson brackets on free associative algebra . 99 


\section{Chapter 1}

\section{Introduction}

A constructive approach to integrability is based upon the study of hidden and rich algebraic or analytic structures associated with integrable equations. In this survey algebraic structures associated with integrable ODEs and PDEs with two independent variables are considered. Some of them are related to Lax representations for differential equations. Furthermore, the bi-Hamiltonian formalism and the AKS factorization method are considered. Structures relevant to Yang-Baxter r-matrix are not discussed since many nice books have been written on the subject (see, for example $[18,63])$.

The statements are formulated in the simplest form but usually possible ways for generalization are pointed out. In the proofs only essential points are mentioned while for technical details references are given. The text contains many carefully selected examples, which give a sense of the subject. A number of open problems are suggested.

The author is not a scrabble in original references. Instead, some references to reviews, where an information of pioneer works can be found, are given.

The survey is addressed to both experts in algebra and in classical integrable systems. It is accessible to $\mathrm{PhD}$ students and can serve as an introduction to classical integrability for scientists with algebraic inclinations.

The exposition is based on a series of lectures delivered by the author in USP (Sao Paulo, 2015).

The contribution of my collaborators I. Golubchik, V. Drinfeld, and A. Odesskii to results presented in this survey is difficult to overestimate.

The author is thankful to the first readers of the survey A. Zobnin and S. Carpentier who made many suggestions and found a lot of misprints, contributing to the improvement of the text.

The author is grateful to V. Kac, I. Shestakov and V. Futorny for their 
attention and to FAPESP for the financial support (grants 2014/002462 and 2016/07265-8) of my visits to Brazil, where the survey has been written.

\subsection{List of basic notation}

\subsubsection{Constants, vectors and matrices}

Henceforth, the field of constants is $\mathbb{C} ; \mathbf{u}$ stands for $N$-dimensional vector, namely $\mathbf{u}=\left(u^{1}, \ldots, u^{N}\right)$. Moreover, the standard scalar product $\sum_{i=1}^{N} u^{i} v^{i}$ is denoted by $\langle\mathbf{u}, \mathbf{v}\rangle$.

The associative algebra of order " $\mathrm{m}$ " square matrices is denoted by Mat $_{m}$; the matrix $\left\{u_{i j}\right\} \in$ Mat $_{m}$ is denoted by $\mathbf{U}$. The unity matrix is denoted by $\mathbf{1}$ or $\mathbf{1}_{m}$. The notation $\mathbf{U}^{t}$ stands for the matrix transpose of U.

For the set of $n \times m$ matrices we use the notation Mat $_{n, m}$.

\subsubsection{Derivations and differential operators}

For ODEs the independent variable is denoted by $t$, whereas for PDEs we have two independent variables $t$ and $x$. Notation $u_{t}$ stands for the partial derivative of $u$ with respect to $t$. For the $x$-partial derivatives of $u$ the notation $u_{x}=u_{1}, u_{x x}=u_{2}$, etc, is used.

The operator $\frac{d}{d x}$ is often denoted by $D$. For the differential operator $L=\sum_{i=0}^{k} a_{i} D^{i}$ we define the operator $L^{+}$as

$$
L^{+}=\sum_{i=0}^{k}(-1)^{i} D^{i} \circ a_{i}
$$

where $\circ$ means that, in this formula, $a_{i}$ is the operator of multiplication by $a_{i}$. By $L_{t}$ we denote

$$
L_{t}=\sum_{i=0}^{k}\left(a_{i}\right)_{t} D^{i} .
$$

\subsubsection{Differential algebra}

We denote by $\mathcal{F}$ a differential field. For our main considerations one can assume that elements of $\mathcal{F}$ are rational functions of a finite number of independent variables $u_{i}$. However, very often we find some functions solving overdetermined systems of PDEs. In such a case we have to extend the basic field $\mathcal{F}$. We will avoid any formal description of such extensions hoping that in any particular case it will be clear what we really need from $\mathcal{F}$. 
The principle derivation

$$
D \stackrel{\text { def }}{=} \frac{\partial}{\partial x}+\sum_{i=0}^{\infty} u_{i+1} \frac{\partial}{\partial u_{i}}
$$

generates all independent variables $u_{i}$ starting from $u_{0}=u$.

When we speak of solutions (or common solutions) of ODEs and PDEs, we mean local solutions with generic initial date.

\subsubsection{Algebra}

We denote by $A(\circ)$ an $N$-dimensional algebra $A$ over $\mathbb{C}$ with an operation $\circ$. A basis of $A$ is denoted by $\mathbf{e}_{1}, \ldots, \mathbf{e}_{N}$, and corresponding structural constants by $C_{j k}^{i}$ :

$$
\mathbf{e}_{j} \circ \mathbf{e}_{k}=C_{j k}^{i} \mathbf{e}_{i}
$$

In what follows we assume that the summation is carried out over repeated indices. We will use the following notation:

$$
\begin{aligned}
A s(X, Y, Z) & =(X \circ Y) \circ Z-X \circ(Y \circ Z), \\
{[X, Y, Z] } & =A s(X, Y, Z)-A s(Y, X, Z) .
\end{aligned}
$$

By $\mathcal{G}$ and $\mathcal{A}$ we usually denote a Lie and an associative algebra, respectively.

The algebra of Laurent series of the form

$$
S=\sum_{i=-n}^{\infty} c_{i} \lambda^{i}, \quad c_{i} \in \mathbb{C}, \quad n \in \mathbb{Z}
$$

is denoted by $\mathbb{C}((\lambda))$, for the subalgebra of Taylor series we use $\mathbb{C}[[\lambda]]$ and $\mathbb{C}[\lambda]$ stands for polynomials in $\lambda$. By $S_{+}$and $S_{-}$we denote

$$
S_{+}=\sum_{i=0}^{\infty} c_{i} \lambda^{i}, \quad \text { and } \quad S_{-}=\sum_{i=-n}^{-1} c_{i} \lambda^{i},
$$

respectively. We use a similar notation for the commutative and noncommutative Laurent series with coefficients from Lie and associative algebras.

\subsection{Lax pairs}

The modern theory of integrable systems was inspired by the discovery of the inverse transform method [54], [1, Chapter 1]. The main ingredient 
of this method is a Lax representation for a differential equation under investigation.

A Lax representation for a given differential equation is a relation of the form

$$
L_{t}=[A, L],
$$

where $L$ and $A$ are some operators, which is equivalent to the differential equation. To apply the technique of the inverse scattering method the operators $L$ and $A$ should depend on an additional (complex) parameter $\lambda$.

\section{ODE case}

A Lax representation for a differential equation

$$
\mathbf{u}_{t}=\mathbf{F}(\mathbf{u}), \quad \mathbf{u}=\left(\mathrm{u}^{1}, \ldots, \mathrm{u}^{\mathrm{N}}\right)
$$

is a relation of the form (1.4), where $L=L(\mathbf{u}, \lambda), A=A(\mathbf{u}, \lambda)$ are some matrices.

Lemma 1.1. i) If $L_{1}$ and $L_{2}$ satisfy (1.4), then $L=L_{1} L_{2}$ satisfies (1.4);

ii) $\bar{L}=L^{n}$ satisfies (1.4) for any $n \in \mathbb{N}$;

iii) $\operatorname{tr} L^{n}$ is an integral of motion for (1.5);

$i \mathrm{v})$ the coefficients of the characteristic polynomial Det $(L-\mu \mathbf{1})$ are integrals of motion.

Proof. Item i). We have

$$
L_{t}=\left(L_{1}\right)_{t} L_{2}+L_{1}\left(L_{2}\right)_{t}=\left[A, L_{1}\right] L_{2}+L_{1}\left[A, L_{2}\right]=A L-L A .
$$

Item ii) follows from Item i). Item iii): if we apply the trace functional to both sides of the identity $\left(L^{n}\right)_{t}=\left[A, L^{n}\right]$, we get $\left(\operatorname{tr} L^{n}\right)_{t}=0$. Item iv) follows from Item ii) and from the formula

$$
\operatorname{Det}(L-\mu \mathbf{1})=\exp (\operatorname{tr}(\log (L-\mu \mathbf{1}))) .
$$

Example 1.1. [46] Let $\mathbf{U}(t)$ be an $m \times m$-matrix,

$$
L=a \lambda+\mathbf{U}, \quad A=\frac{\mathbf{U}^{2}}{\lambda},
$$

where $a=\operatorname{diag}\left(a_{1}, \ldots, a_{m}\right)$. Then (1.4) is equivalent to the ODE

$$
\mathbf{U}_{t}=\left[\mathbf{U}^{2}, a\right] .
$$


If

$$
\mathbf{U}=\left(\begin{array}{ccc}
0 & u_{1} & u_{2} \\
-u_{1} & 0 & u_{3} \\
-u_{2} & -u_{3} & 0
\end{array}\right), \quad a=\left(\begin{array}{ccc}
a_{3} & 0 & 0 \\
0 & a_{2} & 0 \\
0 & 0 & a_{1}
\end{array}\right)
$$

where $a_{i}$ are arbitrary parameters, then (1.6) is equivalent to the Euler top

$\left(u_{1}\right)_{t}=\left(a_{3}-a_{2}\right) u_{2} u_{3}, \quad\left(u_{2}\right)_{t}=\left(a_{1}-a_{3}\right) u_{1} u_{3}, \quad\left(u_{3}\right)_{t}=\left(a_{2}-a_{1}\right) u_{1} u_{2}$.

The characteristic polynomial Det $(L-\mu \mathbf{1})$ is given by

$$
\left(\mu-a_{1} \lambda\right)\left(\mu-a_{2} \lambda\right)\left(\mu-a_{3} \lambda\right)+\left(u_{1}^{2}+u_{2}^{2}+u_{3}^{2}\right) \mu+\left(a_{1} u_{1}^{2}+a_{2} u_{2}^{2}+a_{3} u_{3}^{2}\right) \lambda .
$$

The coefficients of the monomials in $\lambda$ and $\mu$ provide two non-trivial first integrals for the Euler top. The corresponding characteristic curve

$$
\operatorname{Det}(L-\mu \mathbf{1})=0
$$

is elliptic. The eigenfunction $\Psi(\lambda, \mu, t)$ satisfying

$$
L \Psi=\mu \Psi
$$

defines a vector bundle over this curve. The dependence $\Psi$ on $t$ is described by the linear equation

$$
\Psi_{t}=A \Psi .
$$

Using (1.7) and (1.8), one can construct $\Psi(t)$ and after that find the corresponding solution $\mathbf{U}(t)$.

The assumption that $L$ and $A$ in (1.4) are functions of $t$ and $\lambda$ with values in a finite-dimensional Lie algebra $\mathcal{G}$ is a remarkable specification in the case of generic matrices $L$ and $A$, which reduces the number of unknown functions in the corresponding non-linear system of ODEs.

Remark 1.1. We may assume also that in (1.4) the A-operator belongs to $\mathcal{G}$ while $L$ belongs to a module over $\mathcal{G}$ (see, for example, [28]).

\section{Lax pairs for evolution PDEs}

Example 1.2. The Lax pair (1.4) for the $\mathrm{KdV}$ equation

$$
u_{t}=u_{x x x}+6 u u_{x}
$$

found by P. Lax in [43] is given by

$$
L=D^{2}+u+\lambda, \quad A=4 D^{3}+6 u D+3 u_{x}, \quad D=\frac{d}{d x} .
$$

In contrast with Example 1.1, here $L$ and $A$ are differential operators. The relations $(1.7),(1.8)$ allow to construct $\Psi(x, t)$ by the inverse scattering method and, as a result, to find the corresponding solution $u(x, t)$ for the KdV equation. 
Example 1.3. The Lax representation for the nonlinear Schrödinger (NLS) equation written as a system of two equations

$$
u_{t}=-u_{x x}+2 u^{2} v, \quad v_{t}=v_{x x}-2 v^{2} u
$$

has been found by V. Zakharov and A. Shabat [88]. The Lax L-operator is defined by

$$
L=D+\lambda\left(\begin{array}{cc}
1 & 0 \\
0 & -1
\end{array}\right)+\left(\begin{array}{ll}
0 & u \\
v & 0
\end{array}\right) .
$$

The operator $A$ is a polynomial in $\lambda$ with matrix coefficients which depend on $u, v, u_{x}, v_{x}, u_{x x}, v_{x x}, \ldots$ (see Section 2.2).

In this example the $L$-operator has the form $L=D-B$, where $B$ is a matrix depending on unknown functions and the spectral parameter $\lambda$. For this special case equation (1.4) can be written as

$$
A_{x}-B_{t}=[A, B]
$$

Relation (1.11) is called a zero-curvature representation.

In contrast with the ODE case (see Remark 1.1) we may additionally assume only that $A$ and $B$ in (1.11) are functions of $x, t, \lambda$ with values in a finite-dimensional Lie algebra $\mathcal{G}$. In the NLS case we have $\mathcal{G}=\mathfrak{s l}_{2}$.

\subsection{Hamiltonian structures}

Let $y_{1}, \ldots, y_{m}$ be coordinate functions. Any Poisson bracket between functions $f\left(y_{1}, \ldots, y_{m}\right)$ and $g\left(y_{1}, \ldots, y_{m}\right)$ is given by

$$
\{f, g\}=\sum_{i, j} P_{i, j}\left(y_{1}, \ldots, y_{m}\right) \frac{\partial f}{\partial y_{i}} \frac{\partial g}{\partial y_{j}},
$$

where $P_{i, j}=\left\{y_{i}, y_{j}\right\}$. The functions $P_{i j}$ are not arbitrary since by definition

$$
\begin{gathered}
\{f, g\}=-\{g, f\}, \\
\{\{f, g\}, h\}+\{\{g, h\}, f\}+\{\{h, f\}, g\}=0 .
\end{gathered}
$$

Formula (1.12) can be rewritten as

$$
\{f, g\}=\langle\operatorname{grad} f, \mathcal{H}(\operatorname{grad} g)\rangle,
$$

where $\mathcal{H}=\left\{P_{i, j}\right\}$ and $\langle\cdot, \cdot\rangle$ is the standard scalar product. $\mathcal{H}$ is called a Hamiltonian operator or a Poisson tensor.

Definition 1.1. The Poisson bracket is called degenerate if Det $\mathcal{H}=0$. 
The Hamiltonian dynamics is defined by

$$
\frac{d y_{i}}{d t}=\left\{H, y_{i}\right\}, \quad i=1, \ldots, m
$$

where $H$ is a Hamiltonian function. If $\{K, H\}=0$, then $K$ is an integral of motion for the dynamical system. In this case the dynamical system

$$
\frac{d y_{i}}{d \tau}=\left\{K, y_{i}\right\}
$$

is an infinitesimal symmetry for (1.16) [60].

If $\{J, f\}=0$ for any $f$, then $J$ is called a Casimir function of the Poisson bracket. The Casimir functions exist iff the bracket is degenerate.

For the symplectic manifold the coordinates are denoted by $q_{i}$ and $p_{i}$, $i=1, \ldots N$. The standard constant Poisson bracket is given by

$$
\left\{p_{i}, p_{j}\right\}=\left\{q_{i}, q_{j}\right\}=0, \quad\left\{p_{i}, q_{j}\right\}=\delta_{i, j},
$$

where $\delta$ is the Kronecker symbol. The corresponding dynamical system has the usual Hamiltonian form

$$
\frac{d p_{i}}{d t}=-\frac{\partial H}{\partial q_{i}}, \quad \frac{d q_{i}}{d t}=\frac{\partial H}{\partial p_{i}} .
$$

For linear Poisson brackets we have

$$
P_{i j}=\sum_{k} b_{i j}^{k} x_{k}, \quad i, j, k=1, \ldots, N
$$

It is well-known that this formula defines a Poisson bracket iff $b_{i j}^{k}$ are structure constants of a Lie algebra. Very often the title of this Lie algebra is also used for the corresponding linear Poisson bracket.

For the spinning top-like systems $[6,4]$ the Hamiltonian structure is defined by linear Poisson brackets.

Example 1.4. For the models of rigid body dynamics [6] the Poisson bracket is given by

$$
\left\{M_{i}, M_{j}\right\}=\varepsilon_{i j k} M_{k}, \quad\left\{M_{i}, \gamma_{j}\right\}=\varepsilon_{i j k} \gamma_{k}, \quad\left\{\gamma_{i}, \gamma_{j}\right\}=0
$$

Here $M_{i}$ and $\gamma_{i}$ are components of 3 -dimensional vectors $\mathbf{M}$ and $\boldsymbol{\Gamma}, \varepsilon_{i j k}$ is the totally skew-symmetric tensor. The corresponding Lie algebra $e(3)$ is the Lie algebra of the group of motions in $\mathbb{R}^{3}$. This bracket has two Casimir functions

$$
J_{1}=\langle\mathbf{M}, \boldsymbol{\Gamma}\rangle, \quad J_{2}=|\boldsymbol{\Gamma}|^{2}
$$


The class of quadratic Poisson brackets

$$
\left\{x_{i}, x_{j}\right\}=\sum_{p, q} r_{i, j}^{p, q} x_{p} x_{q}, \quad i, j=1, \ldots, N,
$$

is of a great importance for the modern mathematical physics.

As for evolution PDEs of the form

$$
u_{t}=F\left(u, u_{x}, u_{x x}, \ldots, u_{n}\right), \quad u_{i}=\frac{\partial^{i} u}{\partial x^{i}},
$$

the Poisson brackets are also defined by formula (1.15). However, we should take the variational derivative instead of the gradient. Furthermore, the Hamiltonian operator $\mathcal{H}$ is not a matrix but a differential (or even pseudo-differential) operator.

\subsection{Bi-Hamiltonian formalism}

Definition 1.2. [44] Two Poisson brackets $\{\cdot, \cdot\}_{1}$ and $\{\cdot, \cdot\}_{2}$ are said to be compatible if

$$
\{\cdot, \cdot\}_{\lambda}=\{\cdot, \cdot\}_{1}+\lambda\{\cdot, \cdot\}_{2}
$$

is a Poisson bracket for any $\lambda$.

General results on the structure of the Hamiltonian pencil $\{\cdot, \cdot\}_{\lambda}$ can be found in $[45,24]$. In particular, if the bracket $\{\cdot, \cdot\}_{\lambda}$ is degenerate, then a set of commuting integrals can be constructed as follows.

Theorem 1.1. [44] Let

$$
C(\lambda)=C_{0}+\lambda C_{1}+\lambda^{2} C_{2}+\cdots
$$

be a Casimir function for the bracket $\{\cdot, \cdot\}_{\lambda}$. Then the coefficients $C_{i}$ commute with each other with respect to both brackets $\{\cdot, \cdot\}_{1}$ and $\{\cdot, \cdot\}_{2}$.

It follows from Theorem 1.1 that

$$
\left\{C_{k+1}, y\right\}_{1}=-\left\{C_{k}, y\right\}_{2}
$$

for any function $y$ and any $k$. Let us take $C_{k+1}$ for a Hamiltonian $H$. Then the dynamical system (1.16) can be written in two different ways:

$$
\frac{d y_{i}}{d t}=\left\{C_{k+1}, y_{i}\right\}_{1}=-\left\{C_{k}, y_{i}\right\}_{2}
$$

All functions $C_{j}$ are integrals of motion for this system.

We see that the same dynamical system can be represented in two different Hamiltonian forms, with different compatible Hamiltonian structures and different Hamiltonians $C_{k+1}$ and $-C_{k}$. In this case we say that this system possesses a bi-Hamiltonian representation [44].

The spinning top-like systems usually are bi-Hamiltonian with respect to two compatible linear Poisson brackets. The corresponding algebraic object is a pair of compatible Lie algebras (see Section 3.2). 


\subsubsection{Shift argument method}

Here is a standard way of constructing compatible Poisson brackets.

Let $\mathbf{a}=\left(a_{1}, \ldots, a_{N}\right)$ be a constant vector. Then any linear Poisson bracket produces a constant bracket compatible with the initial linear one by the transformation $x_{i} \rightarrow x_{i}+\lambda a_{i}$ (see $[46,52]$ ).

Consider now quadratic Poisson brackets (1.18). The shift $x_{i} \mapsto x_{i}+\lambda a_{i}$ leads to a Poisson bracket of the form $\{\cdot, \cdot\}_{\lambda}=\{\cdot, \cdot\}+\lambda\{\cdot, \cdot\}_{1}+\lambda^{2}\{\cdot, \cdot\}_{2}$. If the coefficient of $\lambda^{2}$ equals zero, then this formula defines a linear Poisson bracket $\{\cdot, \cdot\}_{1}$ compatible with (1.18).

Thus, in the case of quadratic brackets the shift vector $\mathbf{a}=\left(a_{1}, \ldots, a_{N}\right)$ is not arbitrary. Its components have to satisfy the overdetermined system of algebraic equations

$$
\sum_{p, q} r_{i, j}^{p, q} a_{p} a_{q}=0, \quad i, j=1, \ldots, N .
$$

Such a vector a is called admissible. The admissible vectors are nothing but 0-dimensional symplectic leafs for the Poisson bracket (1.18).

Any $p$-dimensional vector space of admissible vectors generates $p$ pairwise compatible linear Poisson brackets. Each of them is compatible with the initial quadratic bracket (1.18).

Many interesting integrable models can be obtained $[38,56,74]$ by the shift argument method from the elliptic quadratic Poisson brackets [20].

Theorem 1.2. For the quadratic Poisson bracket $q_{m n^{2}, k m n-1}(\tau)$ (for the definition of these brackets see [20]), the set of admissible vectors is a union of $n^{2}$ components which are $m$-dimensional vector spaces. The space of generators of the algebra is the direct sum of these spaces.

Open problem 1.1. Find integrable systems generated by the shift argument method from the elliptic Poisson brackets $q_{m n^{2}, k m n-1}(\tau)$.

\subsubsection{Bi-Hamiltonian form for $\mathrm{KdV}$ equation}

Most of known integrable equations (1.19) can be written in a Hamiltonian form

$$
u_{t}=\mathcal{H}\left(\frac{\delta \rho}{\delta u}\right),
$$

where $\mathcal{H}$ is a Hamiltonian operator. The corresponding Poisson bracket is given by

$$
\{f, g\}=\frac{\delta f}{\delta u} \mathcal{H}\left(\frac{\delta g}{\delta u}\right)
$$

where

$$
\frac{\delta}{\delta u}=\sum_{k}(-1)^{k} D^{k} \circ \frac{\partial}{\partial u_{k}}
$$


is the Euler operator or the variational derivative.

Definition 1.3. Two functions $\rho_{1}, \rho_{2}$ are called equivalent $\rho_{1} \sim \rho_{2}$ if $\rho_{1}-\rho_{2} \in \operatorname{Im} D$.

Remark 1.2. For functions $u(x)$ which are rapidly decreasing at $x \rightarrow$ $\pm \infty$, two equivalent polynomial conserved densities $\rho_{1}$ and $\rho_{2}$ with zero constant terms define the same functional

$$
\int_{-\infty}^{+\infty} \rho\left(u, u_{x}, \ldots\right) d x
$$

Proposition 1.1. If $a \in \operatorname{Im} D$, then

$$
\frac{\delta a}{\delta u}=0
$$

and therefore the variational derivative is well defined on the equivalent classes.

By definition the Poisson bracket (1.21) is defined on the vector space of equivalence classes and satisfies (1.13), (1.14). The finite-dimensional bracket (1.12) satisfies also the Leibniz rule

$$
\{f, g h\}=\{f, g\} h+g\{f, h\} .
$$

For brackets (1.21) the Leibniz rule has no sense since the product of equivalence classes is not defined.

We don't discuss here the bi-Hamiltonian formalism for evolution equations of the form (1.19) in general. Notice only that $\mathrm{KdV}$ equation (1.9) is a bi-Hamiltonian system [44]. Two compatible Poisson brackets are given by the formula (1.21), where the Hamiltonian operators $\mathcal{H}_{i}$ are differential ones:

$$
\mathcal{H}_{1}=D, \quad \mathcal{H}_{2}=D^{3}+4 u D+2 u_{x}
$$

Notice that $\mathcal{H}_{1}$ can be obtained from $\mathcal{H}_{2}$ by the argument shift $u \rightarrow u+\lambda$.

The KdV equation can be written in the bi-Hamiltonian form:

$$
u_{t}=\mathcal{H}_{1} \frac{\delta \rho_{1}}{\delta u}=\mathcal{H}_{2} \frac{\delta \rho_{2}}{\delta u},
$$

where

$$
\rho_{1}=-\frac{u_{x}^{2}}{2}+u^{3}, \quad \rho_{2}=\frac{u^{2}}{2} .
$$




\section{Chapter 2}

\section{Factorization of Lie algebras and Lax pairs}

In this chapter we discuss different types of Lax representations for integrable PDEs and some constructions that allow one to find higher symmetries and conservation laws using Lax pairs. For Hamiltonian structures related to Lax operators see, for example, the books $[18,63]$ and the original papers $[12,41]$.

\subsection{Scalar Lax pairs for evolution equations}

In this section the Lax $L$-operators are linear differential operators or ratios of linear differential operators. The corresponding $A$-operators are constructed by the use of formal non-commutative "pseudo-differential" series.

For our purporses the language of differential algebra [36] is the most adequate one.

Consider evolution equations of the form (1.19). Suppose that the right hand side of (1.19) as well as other functions in $u, u_{x}, u_{x x}, \ldots$ belong to a differential field $\mathcal{F}$. For main considerations one can assume that elements of $\mathcal{F}$ are rational functions of finite number of independent variables

$$
u_{i}=\frac{\partial^{i} u}{\partial x^{i}} .
$$

In order to integrate a function with respect to one of its arguments or to take one of its roots, we sometimes have to extend the basic field $\mathcal{F}$. that in any particular case it is clear (to the reader) how to construct them.

As usual in differential algebra, we have a principle derivation (1.1), which generates all independent variables $u_{i}$ starting from $u_{0}=u$. This 
derivation is a formalization of the total $x$-derivative, which acts on functions of the form $g\left(x, u(x), \frac{\partial u}{\partial x}, \ldots\right)$.

The variable $t$ in the local algebraic theory of evolution equations plays the role of a parameter.

A higher (or generalized) infinitesimal symmetry (or a commuting flow) for (1.19) is an evolution equation

$$
u_{\tau}=G\left(u, u_{x}, u_{x x}, \ldots, u_{m}\right), \quad m>1
$$

which is compatible with (1.19). Compatibility means that

$$
\frac{\partial}{\partial t} \frac{\partial u}{\partial \tau}=\frac{\partial}{\partial \tau} \frac{\partial u}{\partial t}
$$

where the partial derivatives are calculated in virtue of (1.19) and (2.1). In other words, for any initial value $u_{0}(x)$ there exists a common solution $u(x, t, \tau)$ of equations (1.19) and (2.1) such that $u(x, 0,0)=u_{0}(x)$.

Example 2.1. The simplest higher symmetry for the Korteweg-de Vries equation (1.9) has the following form

$$
u_{\tau}=u_{5}+10 u u_{3}+20 u_{1} u_{2}+30 u^{2} u_{1} .
$$

The infinite-dimensional vector field

$$
D_{F}=F \frac{\partial}{\partial u_{0}}+D(F) \frac{\partial}{\partial u_{1}}+D^{2}(F) \frac{\partial}{\partial u_{2}}+\cdots
$$

is associated with evolution equation (1.19). This vector field commutes with $D$. We shall call vector fields of the form (2.3) evolutionary. The function $F$ is called generator of that evolutionary vector field. Sometimes we will call (2.3) total $t$-derivative with respect to (1.19) and denote it by $D_{t}$. The set of all evolutionary vector fields form a Lie algebra over $\mathbb{C}$ : $\left[D_{G}, D_{H}\right]=D_{K}$, where

$$
K=D_{G}(H)-D_{H}(G)=H_{*}(G)-G_{*}(H) .
$$

Here and in the sequel we use the following notation:

Definition 2.1. For any element $a \in \mathcal{F}$ the Fréchet derivative $a_{*}$ is a linear differential operator defined by

$$
a_{*} \stackrel{\text { def }}{=} \sum_{k} \frac{\partial a}{\partial u_{k}} D^{k} .
$$

We defined a generalized symmetry of equation (1.19) as an evolution equation (2.1) that is compatible with (1.19). By definition, the compatibility means that $\left[D_{F}, D_{G}\right]=0$. It can also be written in the form

$$
G_{*}(F)-F_{*}(G)=D_{t}(G)-F_{*}(G)=0 .
$$


Formula (2.4) defines a Lie bracket on our differential field $\mathcal{F}$. The integrable hierarchy is nothing but an infinite-dimensional commutative subalgebra of this Lie algebra.

A local conservation law for equation (1.19) is a pair of functions $\rho\left(u, u_{x}, \ldots\right)$ and $\sigma\left(u, u_{x}, \ldots\right)$ such that

$$
D_{t}\left(\rho\left(u, u_{x}, \ldots, u_{p}\right)\right)=D\left(\sigma\left(u, u_{x}, \ldots, u_{q}\right)\right)
$$

for any solution $u(x, t)$ of equation (1.19). The functions $\rho$ and $\sigma$ are called density and flux of the conservation law (2.5). It is easy to see that $q=p+n-1$, where $n$ is the order of equation (1.19).

Example 2.2. Functions

$$
\rho_{1}=u, \quad \rho_{2}=u^{2}, \quad \rho_{3}=-u_{x}^{2}+2 u^{3}
$$

are conserved densities of the Korteweg-de Vries equation (1.9). Indeed,

$$
\begin{aligned}
D_{t}(u) & =D\left(u_{2}+3 u^{2}\right), \\
D_{t}\left(u^{2}\right) & =D\left(2 u u_{x x}-u_{x}^{2}+4 u^{3}\right), \\
D_{t}\left(-u_{x}^{2}+2 u^{3}\right) & =D\left(9 u^{4}+6 u^{2} u_{x x}+u_{x x}^{2}-12 u u_{x}^{2}-2 u_{x} u_{3}\right) .
\end{aligned}
$$

For solitonic type solutions $u(x, t)$ of (1.19), which are decreasing at $x \rightarrow \pm \infty$, it follows from (2.5) that

$$
\frac{\partial}{\partial t} \int_{-\infty}^{+\infty} \rho d x=0 .
$$

This justifies the name conserved density for the function $\rho$. Analogously, if $u$ is a function periodic in space with period $L$, then the value of the functional $I(u)=\int_{0}^{L} \rho d x$ does not depend on time and therefore it is a constant of motion.

Suppose that functions $\rho$ and $\sigma$ satisfy (2.5). Then for any function $s\left(u, u_{x}, \ldots\right)$ the functions $\bar{\rho}=\rho+D(s)$ and $\bar{\sigma}=\sigma+D_{t}(s)$ satisfy $(2.5)$ as well. We call the conserved densities $\rho$ and $\bar{\rho}$ equivalent. It is clear that equivalent densities define the same functional.

\subsubsection{Pseudo-differential series}

Consider a skew field of (non-commutative) formal series of the form

$S=s_{m} D^{m}+s_{m-1} D^{m-1}+\cdots+s_{0}+s_{-1} D^{-1}+s_{-2} D^{-2}+\cdots \quad s_{k} \in \mathcal{F}$. 
The number $m \in \mathbb{Z}$ is called the order of $S$ and is denoted by ord $S$. If $s_{i}=0$ for $i<0$ that $S$ is called a differential operator.

The product of two formal series is defined by the formula

$$
D^{k} \circ s D^{m}=s D^{m+k}+C_{k}^{1} D(s) D^{k+m-1}+C_{k}^{2} D^{2}(s) D^{k+m-2}+\cdots,
$$

where $k, m \in \mathbb{Z}$ and $C_{n}^{j}$ is the binomial coefficient

$$
C_{n}^{j}=\frac{n(n-1)(n-2) \cdots(n-j+1)}{j !}, \quad n \in \mathbb{Z} .
$$

Remark 2.1. For any series $S$ and $T$ we have $\operatorname{ord}(S \circ T-T \circ S) \leq$ ord $S+\operatorname{ord} T-1$.

The formally conjugated formal series $S^{+}$is defined as $S^{+}=(-1)^{m} D^{m} \circ s_{m}+(-1)^{m-1} D^{m-1} \circ s_{m-1}+\cdots+s_{0}-D^{-1} \circ s_{-1}+D^{-2} \circ s_{-2}+\cdots$.

Example 2.3. Let

$$
R=u D^{2}+u_{1} D, \quad S=-u_{1} D^{3}, \quad T=u D^{-1}
$$

then

$$
\begin{gathered}
R^{+}=D^{2} \circ u-D \circ u_{1}=R, \\
S^{+}=D^{3} \circ u_{1}=u_{1} D^{3}+3 u_{2} D^{2}+3 u_{3} D+u_{4}, \\
T^{+}=-D^{-1} u=-u D^{-1}+u_{1} D^{-2}-u_{2} D^{-3}+\cdots .
\end{gathered}
$$

For any series (2.6) one can uniquely find the inverse series

$$
T=t_{-m} D^{-m}+t_{-m-1} D^{-m-1}+\cdots, \quad t_{k} \in \mathcal{F}
$$

such that $S \circ T=T \circ S=1$. Indeed, multiplying $S$ and $T$ and equating the result to 1 , we find that $s_{m} t_{-m}=1$, i. e., $t_{-m}=1 / s_{m}$. Comparing the coefficients of $D^{-1}$, we get

$$
m s_{m} D\left(t_{-m}\right)+s_{m} t_{-m-1}+s_{m-1} t_{-m}=0
$$

and therefore

$$
t_{-m-1}=-\frac{s_{m-1}}{s_{m}^{2}}-m D\left(\frac{1}{s_{m}}\right), \quad \text { etc. }
$$

Furthermore, we can find the $m$-th root of the series $S$, i. e., a series

$$
R=r_{1} D+r_{0}+r_{-1} D^{-1}+r_{-2} D^{-2}+\cdots
$$

such that $R^{m}=S$. This root is unique up to any number factor $\varepsilon$ such that $\varepsilon^{m}=1$. 
Example 2.4. Let $S=D^{2}+u$. Assuming

$$
R=r_{1} D+r_{0}+r_{-1} D^{-1}+r_{-2} D^{-2}+\cdots
$$

we compute

$R^{2}=R \circ R=r_{1}^{2} D^{2}+\left(r_{1} D\left(r_{1}\right)+r_{1} r_{0}+r_{0} r_{1}\right) D+r_{1} D\left(r_{0}\right)+r_{0}^{2}+r_{1} r_{-1}+r_{-1} r_{1}+\cdots$,

and compare the result with $S$. From the coefficients of $D^{2}$ we find $r_{1}^{2}=1$ or $r_{1}= \pm 1$. Let $r_{1}=1$. Comparing coefficients of $D$, we get $2 r_{0}=0$, i. e., $r_{0}=0$. From $D^{0}$ we obtain $2 r_{-1}=u$, terms of $D^{-1} r_{-2}=-u_{1} / 4$, etc., i. e.

$$
R=S^{1 / 2}=D+\frac{u}{2} D^{-1}-\frac{u_{1}}{4} D^{-2}+\cdots .
$$

Definition 2.2. The residue of a formal series (2.6) by definition is the coefficient of $D^{-1}$ :

$$
\operatorname{res}(S) \stackrel{\text { def }}{=} s_{-1} \text {. }
$$

The logarithmic residue of $S$ is defined as

$$
\text { res } \log S \stackrel{\text { def }}{=} \frac{s_{n-1}}{s_{n}}
$$

We will use the following important

Theorem 2.1. [2] For any two formal series $S, T$ the residue of the commutator belongs to $\operatorname{Im} D$ :

$$
\operatorname{res}[S, T]=D(\sigma(S, T))
$$

where

$$
\sigma(S, T)=\sum_{i \leq \operatorname{ord}(T), j \leq \operatorname{ord}(S)}^{i+j+1>0} C_{j}^{i+j+1} \times \sum_{k=0}^{i+j}(-1)^{k} D^{k}\left(s_{j}\right) D^{i+j-k}\left(t_{j}\right) .
$$

\subsubsection{Korteweg-de Vries hierarchy}

For the KdV equation (1.9) the Lax pair is defined by

$$
L=D^{2}+u, \quad A=4\left(D^{3}+\frac{3}{2} u D+\frac{3}{4} u_{x}\right) .
$$

Using these $L$ and $A$ operators, we are going to demonstrate how a scalar differential Lax pair generates higher symmetries, conservation laws and explicit solutions of the solitonic type.

One can easily verify that the commutator $[A, L]$ is equal to the right hand side of (1.9). Since $L_{t}=u_{t}$ the relation (1.4) is equivalent to (1.9). In particular, the commutator $[A, L]$ does not contain any powers of $D$, i. e., it is a differential operator of zero order. 
Problem 2.1. How to describe all differential operators

$$
P_{n}=D^{n}+\sum_{i=0}^{n-1} p_{i} D^{i}
$$

such that $\left[P_{n}, L\right]$ is a differential operator of zero order?

It is clear that for such an operator $P_{n}$ the relation $L_{t}=\left[P_{n}, L\right]$ is equivalent to an evolution equation of the form (1.19).

Definition 2.3. For any series

$$
P=\sum_{i=-\infty}^{k} p_{i} D^{i}
$$

we denote

$$
P_{+}=\sum_{i=0}^{k} p_{i} D^{i}, \quad P_{-}=\sum_{i=-\infty}^{-1} p_{i} D^{i} .
$$

Remark 2.2. We consider a vector space decomposition of the associative algebra of all pseudo-differential series into a direct sum of the subalgebra of differential operators and the subalgebra of series of negative orders. The subscripts + and - symbolize the projections onto these subalgebras.

Lemma 2.1. Let $P$ be a formal series such that $[L, P]=0$; then

$$
\left[P_{+}, L\right]=f, \quad f \in \mathcal{F} .
$$

Proof. Since $\left[L, P_{+}+P_{-}\right]=0$, we have

$$
\left[P_{+}, L\right]=-\left[P_{-}, L\right] .
$$

The left hand side of this identity is a differential operator while according to Remark 2.1 the order of the right hand side is not positive.

Lemma 2.2. The following relation holds:

$$
\left[L, L^{\frac{1}{2}}\right]=0 .
$$

Proof. Let

$$
\left[L, L^{\frac{1}{2}}\right]=\sigma D^{p}+\cdots
$$

Then (see Example 2.4) we have

$$
0=[L, L]=\left[L, L^{\frac{1}{2}}\right] L^{\frac{1}{2}}+L^{\frac{1}{2}}\left[L, L^{\frac{1}{2}}\right]=2 \sigma D^{p+1} \cdots,
$$

and therefore $s=0$. 
Corollary 2.1. It follows from Lemmas 2.1 and 2.2 that for any $n \in \mathbb{Z}_{+}$ the differential operator $P=L_{+}^{\frac{n}{2}}$ satisfies the relation

$$
[P, L]=f_{P}, \quad f_{P} \in \mathcal{F}
$$

for some $f_{P}$. If $n$ is even, then $f_{P}=0$.

It is clear that the set of all differential operators that satisfy (2.9) is a vector space over $\mathbb{C}$.

Lemma 2.3. The differential operators $L_{+}^{\frac{n}{2}}, n \in \mathbb{Z}_{+}$, form a basis of this vector space.

Proof. Suppose that $P=\sigma D^{p}+\cdots$ satisfies (2.9). Equating the coefficients of $D^{p+1}$, we get $D(\sigma)=0$ and therefore $\sigma=$ const. Since the operator $\sigma L_{+}^{\frac{p}{2}}$ has the same leading coefficient as $P$, the operator $P-\sigma L_{+}^{\frac{p}{2}}$ has strictly lower order than $P$. The induction over $p$ completes the proof.

Let

$$
\left[L_{+}^{\frac{n}{2}}, L\right]=f_{n}
$$

For even $n$ we have $f_{n}=0$ and the evolution equation $u_{t}=f_{n}$ that is equivalent to

$$
L_{t}=\left[L_{+}^{\frac{n}{2}}, L\right]
$$

is trivial. Denote

$$
A_{n}=L_{+}^{\frac{2 n+1}{2}} .
$$

It can be easily verified that $A=4 A_{1}$, where $A$ is defined by (2.7). The evolution equation corresponding to $n=0$ is just $u_{t}=u_{x}$.

Theorem 2.2. For any $n, m \in \mathbb{N}$ the evolution equations $u_{\tau}=f_{2 m+1}$ and $u_{t}=f_{2 n+1}$ are compatible ${ }^{1}$.

Proof. Let us rewrite these equations in the Lax form:

$$
L_{t}=\left[A_{n}, L\right], \quad L_{\tau}=\left[A_{m}, L\right]
$$

We have

$$
\left(L_{t}\right)_{\tau}-\left(L_{\tau}\right)_{t}=\left[\left(A_{n}\right)_{\tau}, L\right]-\left[\left(A_{m}\right)_{t}, L\right]+\left[A_{n},\left[A_{m}, L\right]\right]-\left[A_{m},\left[A_{n}, L\right]\right] .
$$

Due to the Jacobi identity it suffices to prove that

$$
\left(A_{n}\right)_{\tau}-\left(A_{m}\right)_{t}+\left[A_{n}, A_{m}\right]=0 .
$$

\footnotetext{
${ }^{1}$ In other words, the first equation is a higher symmetry for the second one and vice versa
} 
Since $\left(L^{p}\right)_{t}=\left[A_{n}, L^{p}\right]$ and $\left(L^{p}\right)_{\tau}=\left[A_{m}, L^{p}\right]$ for any $p$, we get

$$
\left(A_{n}\right)_{\tau}=\left(\left[A_{m}, L^{\frac{2 n+1}{2}}\right]\right)_{+}, \quad\left(A_{m}\right)_{t}=\left(\left[A_{n}, L^{\frac{2 m+1}{2}}\right]\right)_{+} .
$$

Therefore, we need to verify that

$$
\left(\left[A_{m}, L^{\frac{2 n+1}{2}}\right]-\left[A_{n}, L^{\frac{2 m+1}{2}}\right]+\left[A_{n}, A_{m}\right]\right)_{+}=0 .
$$

Substituting

$$
A_{n}=L^{\frac{2 n+1}{2}}-\left(L^{\frac{2 n+1}{2}}\right)_{-}
$$

and

$$
A_{m}=L^{\frac{2 m+1}{2}}-\left(L^{\frac{2 m+1}{2}}\right)_{-}
$$

to the latter identity, we obtain

$$
\left[\left(L^{\frac{2 n+1}{2}}\right)_{-},\left(L^{\frac{2 m+1}{2}}\right)_{-}\right]_{+}=0
$$

which is obviously true.

Corollary 2.2. Any two evolution equations defined by (1.4) with different A-operators of the form

$$
A=\sum_{i \geq 0} c_{i} L_{+}^{\frac{2 i+1}{2}}, \quad c_{i} \in \mathbb{C},
$$

are compatible.

This infinite-dimensional vector space of compatible evolution equations is called the $K d V$ hierarchy [22]. Any two equations of the hierarchy are higher symmetries for each other.

Thus, the symmetries of the KdV equation are generated by the same $L$-operator but by different $A$-operators.

\section{Recursion operator for $\mathrm{KdV}$ equation}

Now we are going to find [35, Section $2 \mathrm{~A}]$ a recursion relation between $f_{2 n+1}$ and $f_{2 n+3}$. A similar method was applied for the first time in [75] to find a recursion operator for the Krichever-Novikov equation.

Since $L^{\frac{2 n+3}{2}}=L L^{\frac{2 n+1}{2}}$, we have

$$
A_{n+1}=\left(L L^{\frac{2 n+1}{2}}\right)_{+}=L\left(L^{\frac{2 n+1}{2}}\right)_{+}+\left(L\left(L^{\frac{2 n+1}{2}}\right)_{-}\right)_{+},
$$

or

$$
A_{n+1}=L A_{n}+R_{n},
$$


where $R_{n}=a_{n} D+b_{n}$ is a differential operator of first order. Hence

$$
f_{2 n+3}=\left[A_{n+1}, L\right]=L \circ f_{2 n+1}+\left[R_{n}, L\right] .
$$

Now if we equate to zero coefficients of $D^{2}, D$ and $D^{0}$ in the above equation, we obtain

$$
a_{n}=\frac{1}{2} D^{-1}\left(f_{2 n+1}\right), \quad b_{n}=\frac{3}{4} f_{2 n+1}
$$

and

$$
f_{2 n+3}=\left(\frac{1}{4} D^{2}+u+\frac{1}{2} u_{x} D^{-1}\right) f_{2 n+1},
$$

which gives the standard recursion operator

$$
\mathcal{R}=\frac{1}{4} D^{2}+u+\frac{1}{2} u_{x} D^{-1}
$$

for the KdV equation

$$
u_{t}=\frac{1}{4}\left(u_{x x x}+6 u u_{x}\right) .
$$

The factor $1 / 4$ appears due to the fact that we take for $A$-operator $L_{+}^{\frac{3}{2}}$ instead of $4 L_{+}^{\frac{3}{2}}$. Of course, this coefficient can be removed by the scaling $t \rightarrow 4 t$.

As it was shown in [35] this method for finding a recursion operator can be generalized to Lax pairs of different type.

Exercise 2.1. Check that the recursion operator (2.11) satisfies the operator identity

$$
\mathcal{R}_{t}=\left[F_{*}, \mathcal{R}\right]
$$

where

$$
F_{*}=\frac{1}{4}\left(D^{3}+6 u D+6 u_{x}\right)
$$

is the Frechét derivative of the right hand side of the KdV equation (2.12). Exercise 2.2. (see [35, Appendix A]) Find the recursion operator for the Boussinesq system

$$
u_{t}=v_{x}, \quad v_{t}=-\frac{1}{3}\left(u_{x x x}+8 u u_{x}\right) .
$$

A Lax pair for this system is given by

$$
L=D^{3}+2 u D+u_{x}+v, \quad A=\left(L^{\frac{2}{3}}\right)_{+} .
$$




\section{Conservation laws}

Proposition 2.1. For any $n \in \mathbb{N}$ the function

$$
\rho_{n}=\operatorname{res}\left(L^{\frac{2 n-1}{2}}\right)
$$

where $L$ is defined by (2.7), is a conserved density for the KdV equation.

Proof. It is easy to prove (cf. Lemma 1.1) that

$$
\left(L^{\frac{2 n-1}{2}}\right)_{t}=\left[A,\left(L^{\frac{2 n-1}{2}}\right)\right] .
$$

Finding residue of both sides of this identity and taking into account Theorem 2.1, we arrive at the statement of the proposition.

It can be verified that formula (2.13) with $n=1,2,3$ produces conserved densities equivalent to the ones presented in Example 2.2.

\section{Darboux transformation}

The Darboux transformation for the $\mathrm{KdV}$ equation is defined by the following relation

$$
\tilde{L}=T L T^{-1}
$$

where

$$
T=D^{n}+a_{n-1} D^{n-1}+\cdots+a_{0}
$$

is a differential operator. In the generic case $\tilde{L}$ is a pseudo-differential series, but for special $T$ this series could be a differential operator of the form $\tilde{L}=D^{2}+\tilde{u}$. In this case we have

$$
\left(D^{n}+a_{n-1} D^{n-1}+\cdots+a_{0}\right)\left(D^{2}+u\right)=\left(D^{2}+\tilde{u}\right)\left(D^{n}+a_{n-1} D^{n-1}+\cdots+a_{0}\right) .
$$

Comparing the coefficients of $D^{n}$, we get

$$
\tilde{u}=u-2 \frac{\partial}{\partial x} a_{n-1}
$$

This formula allows us to construct a new solution $\tilde{u}$ of the KdV equation starting from a given solution $u$.

It follows from (2.15) that

$$
L(\operatorname{Ker} T) \subset \operatorname{Ker} T \text {. }
$$

The existence of the Euclidean algorithm in the ring of differential operators [61] guarantees that $(2.16)$ is a sufficient condition for $\tilde{L}$ to be a differential operator. 
Suppose that the Jordan form of the operator $L$ acting on the finitedimensional space $\operatorname{Ker} T$ is diagonal. Then a basis of $\operatorname{Ker} T$ is given by some functions $\Psi_{1}, \ldots, \Psi_{n}$, such that

$$
\frac{\partial^{2}}{\partial x^{2}} \Psi_{i}+u \Psi_{i}=\lambda_{i}^{2} \Psi_{i}
$$

If the functions $\Psi_{1}, \ldots, \Psi_{n}$ are fixed, then, up to a left factor, the equation $T(Y)=0$ is given by the formula

$$
\mathbf{W}\left(\Psi_{1}, \ldots, \Psi_{n}, Y\right)=0,
$$

where $\mathbf{W}$ is the Wronskian. This implies

$$
a_{n-1}=-\frac{\partial}{\partial x} \log \mathbf{W}\left(\Psi_{1}, \ldots, \Psi_{n}\right) .
$$

and therefore the Darboux transformation has the following form:

$$
\tilde{u}=u+2 \frac{\partial^{2}}{\partial x^{2}} \log \mathbf{W}\left(\Psi_{1}, \ldots, \Psi_{n}\right) .
$$

As usual, the $t$-dynamics of $\Psi_{i}$ is defined by the $A$-operator:

$$
\frac{\partial}{\partial t}\left(\Psi_{i}\right)=A\left(\Psi_{i}\right) \equiv \frac{\partial^{3}}{\partial x^{3}} \Psi_{i}+\frac{3}{2} u \frac{\partial}{\partial x}\left(\Psi_{i}\right)+\frac{3}{4} u_{x} \Psi_{i} .
$$

Theorem 2.3. Let $u(x, t)$ be any solution of the KdV equation (2.12). If functions $\Psi_{i}$ satisfy (2.17) and (2.19), then the function $\tilde{u}(x, t)$ defined by (2.18) satisfies the KdV equation.

Proof. The Lax equation (1.4) can be rewritten in the commutator form $\left[\frac{\partial}{\partial t}-A, L\right]=0$. This implies $\left[\frac{\partial}{\partial t}-\tilde{A}, \tilde{L}\right]=0$, where the differential operator $\tilde{L}$ is defined by $(2.14)$ and

$$
\tilde{A}=T A T^{-1}+T_{t} T^{-1} .
$$

It suffices to check that the ratio of differential operators $T A+T_{t}$ and $T$ is a differential operator. This is equivalent to the fact that

$$
T A\left(\Psi_{i}\right)+T_{t}\left(\Psi_{i}\right)=0
$$

for any $i$. We have $0=\left(T \Psi_{i}\right)_{t}=T_{t} \Psi_{i}+T\left(\Psi_{i}\right)_{t}$. Substituting $-T\left(\Psi_{i}\right)_{t}$ for $T_{t}\left(\Psi_{i}\right)$ into (2.21) and using (2.19), we complete the proof.

Remark 2.3. The numbers $\lambda_{i}$ from (2.17) are arbitrary parameters in the solution (2.18).

Exercise 2.3. Prove that for any Jordan form of the operator $L$ acting on the finite-dimensional space $\operatorname{Ker} T$ the condition

$$
\left(\frac{\partial}{\partial t}-A\right) \operatorname{Ker} T \subset \operatorname{Ker} T
$$

provides the fact that $\tilde{A}$, defined by $(2.20)$, is a differential operator. 


\section{Solitons and rational solutions for $\mathrm{KdV}$ equation}

Let us start from the trivial solution $u(x, t)=0$ of the KdV equation. In this case condition (2.16) means that $\operatorname{Ker} T$ is any finite-dimensional vector space $\mathbf{V}$ of functions invariant with respect to the differential operator $\frac{\partial^{2}}{\partial x^{2}}$. The $t$-dynamics of $\mathbf{V}$ is defined by the condition

$$
\left(\frac{\partial}{\partial t}-\frac{\partial^{3}}{\partial x^{3}}\right) \mathbf{V} \subset \mathbf{V}
$$

In the generic case, when the Jordan form of the operator $\frac{\partial^{2}}{\partial x^{2}}$ is diagonal, a basis of such a vector space is given by

$$
\Psi_{i}(x, t)=\exp \left(\eta_{i}\right)+c_{i} \exp \left(-\eta_{i}\right)
$$

where

$$
\eta_{i}=\lambda_{i} x+\lambda_{i}^{3} t, \quad i=1, \ldots, n
$$

The function

$$
\tilde{u}=2 \frac{\partial^{2}}{\partial x^{2}} \log \mathbf{W}\left(\Psi_{1}, \ldots, \Psi_{n}\right)
$$

is called $n$-soliton solution of the $\mathrm{KdV}$ equation.

Example 2.5. If $n=1$, we have

$$
\tilde{u}(x, t)=\frac{8 c_{1} \lambda_{1}^{2}}{\left(e^{\lambda_{1} x+\lambda_{1}^{3} t}+c_{1} e^{-\lambda_{1} x-\lambda_{1}^{3} t}\right)^{2}}
$$

Example 2.6. The 2-soliton solution for the $\mathrm{KdV}$ equation is given by

$$
\tilde{u}(x, t)=\left(\lambda_{2}^{2}-\lambda_{1}^{2}\right) \frac{\frac{8 c_{1} \lambda_{1}^{2}}{\left(e^{\eta_{1}}+c_{1} e^{-\eta_{1}}\right)^{2}}-\frac{8 c_{2} \lambda_{2}^{2}}{\left(e^{\eta_{2}}+c_{2} e^{-\eta_{2}}\right)^{2}}}{\left(\lambda_{1} \frac{c_{1}-e^{2 \eta_{1}}}{c_{1}+e^{2 \eta_{1}}}-\lambda_{2} \frac{c_{2}-e^{2 \eta_{2}}}{c_{2}+e^{2 \eta_{2}}}\right)^{2}} .
$$

If the vector space $\mathbf{V}$ consists of polynomials in $x$, we get rational solutions of the $\mathrm{KdV}$ equation. In the simplest case $\operatorname{dim} \mathbf{V}=1$ we have $\Psi_{1}=x$ and formula (2.18) produces a stationary rational solution

$$
\tilde{u}(x, t)=-\frac{2}{x^{2}} .
$$

\subsubsection{Gelfand-Dikii hierarchy and generalizations}

Let

$$
L=D^{n}+\sum_{i=0}^{n-2} u_{i} D^{i}, \quad A=\sum_{i=0}^{m} c_{i} L_{+}^{\frac{i}{n}}, \quad c_{i} \in \mathbb{C}
$$


In the same way as in Lemmas 2.1 and 2.3 it can be proved that the Lax equation (1.4) is equivalent to a system of $n-1$ evolution equations for unknown functions $u_{n-2}, \ldots, u_{0}$. Moreover, the systems generated by the same $L$-operator and $A$-operators of the form (2.23) with different $m$ and $c_{i}$ are higher symmetries for each other. This infinite set of compatible evolution systems is called the Gelfand-Dikii hierarchy [23]. If $n=2$, we get the KdV hierarchy described above.

\section{Factorization of $L$-operator}

Relations between factorization of scalar differential Lax operators, Miuratype transformations and modified $\mathrm{KdV}$-type systems were discussed, for example, in $[77,21,12]$. We are concerned here with the case of two factors only.

Consider the following system of the Lax type equations

$$
M_{t}=A N-M B, \quad N_{t}=B N-N A,
$$

where

$$
\begin{array}{ll}
M=D^{r}+w D^{r-1}+\sum_{i=0}^{r-2} u_{i} D^{i}, & N=D^{s}-w D^{s-1}+\sum_{i=0}^{s-2} v_{i} D^{i}, \\
A=\sum_{i=0}^{m} c_{i}\left((M N)^{\frac{i}{r+s}}\right)_{+}, & B=\left(M^{-1} A M\right)_{+} .
\end{array}
$$

System (2.24) is related to Lax equation (1.4). Namely, if $M$ and $N$ satisfy (2.24), then $L=M N$ satisfies (1.4).

Proposition 2.2. Relations (2.24) are equivalent to a system of $r+s-1$ evolution equations in $w, u_{i}, v_{i}$.

\section{Reductions in differential $L$-operators}

Let us introduce the following notation:

$$
\begin{aligned}
& Q_{1}(n) \stackrel{\text { def }}{=} D^{2 n+1}+\sum_{i=0}^{n-1} u_{i} D^{2 i+1}+D^{2 i+1} u_{i}, \\
& Q_{2}(n) \stackrel{\text { def }}{=} D^{2 n}+\sum_{i=0}^{n-1} u_{i} D^{2 i}+D^{2 i} u_{i}, \\
& Q_{3}(n) \stackrel{\text { def }}{=} D^{2 n-1}+\sum_{i=1}^{n-1} u_{i} D^{2 i-1}+D^{2 i-1} u_{i}+u_{0} D^{-1} u_{0}
\end{aligned}
$$

We call $u_{n-1}, \ldots, u_{0}$ functional parameters of $Q_{i}(n)$. By definition, we put

$$
Q_{1}(0) \stackrel{\text { def }}{=} D, \quad Q_{2}(0) \stackrel{\text { def }}{=} 1, \quad Q_{3}(0) \stackrel{\text { def }}{=} D^{-1}
$$


Notice that the operators $Q_{1}(n)$ and $Q_{3}(n)$ are skew-symmetric: $Q_{1}(n)^{+}=$ $-Q_{1}(n)$ and $Q_{3}(n)^{+}=-Q_{3}(n)$. The operators $Q_{2}(n)$ are symmetric: $Q_{2}(n)^{+}=Q_{2}(n)$.

There are deep relations between such operators and classical simple Lie algebras [12, Section 7]. The algebra $B_{n}$ corresponds to an operator of $Q_{1}(n)$-type, while the algebras $C_{n}$ and $D_{n}$ correspond to operators of types $Q_{2}(n)$ and $Q_{3}(n)$.

Theorem 2.4. (see [12, Section 7], [13]) Suppose the operators $M=$ $Q_{i}(r)$ and $N=Q_{j}(s)$, where $i, j \in\{1,2,3\}$, have functional parameters $u_{r-1}, \ldots, u_{0}$ and $v_{s-1}, \ldots, v_{0}$, respectively. Then relations (2.24), where

$$
A=\sum_{i=0}^{m} c_{i}\left(L^{\frac{2 i+1}{n}}\right)_{+}, \quad B=\left(M^{-1} A M\right)_{+},
$$

$L=M N$ and $n=\operatorname{ord} L$, are equivalent to a system of $r+s$ evolution equations in $u_{r-1}, \ldots, u_{0}, v_{s-1}, \ldots, v_{0}$.

Here we present several examples [13] with $r+s \leq 2$, where the operator $A$ has a minimal possible order. In the corresponding differential equations we perform some scalings of independent variables and unknown functions to reduce equations to a simple form. If any different transformations were applied, then we point out their form up to constants, which can be easily reconstructed by reader.

Example 2.7. In the cases
a) $\quad L=D^{2}+u, \quad A=\left(L^{\frac{3}{2}}\right)_{+}$,
b) $\quad L=\left(D^{2}+u\right) D^{-1}, \quad A=\left(L^{3}\right)_{+}$,
c) $\quad L=\left(D^{3}+2 u D+u_{x}\right) D^{-1}, \quad A=\left(L^{\frac{3}{2}}\right)_{+}$,
d) $\quad L=\left(D^{3}+2 u D+u_{x}\right) D, \quad A=\left(L^{\frac{3}{4}}\right)_{+}$

we get the KdV equation (1.9).

Example 2.8. The cases
a)

$$
L=D+u D^{-1} u
$$$$
A=\left(L^{3}\right)_{+},
$$
b) $\quad L=\left(D+u D^{-1} u\right) D$,

$$
A=\left(L^{\frac{3}{2}}\right)_{+}
$$

give rise to the modified $\mathrm{KdV}$ equation

$$
u_{t}=u_{x x x}+6 u^{2} u_{x} .
$$


Example 2.9. The operators

$$
L=\left(D^{2}+u\right) D, \quad A=\left(L^{\frac{5}{3}}\right)_{+}
$$

produce the Savada-Kotera equation [66]

$$
u_{t}=u_{5}+5 u u_{3}+5 u_{1} u_{2}+5 u^{2} u_{1} .
$$

Example 2.10. In the case

$$
L=D^{3}+2 u D+u_{x}, \quad A=\left(L^{\frac{5}{3}}\right)_{+}
$$

we obtain the Kaup-Kupershmidt equation [37]

$$
u_{t}=u_{5}+10 u u_{3}+25 u_{1} u_{2}+20 u^{2} u_{1} .
$$

Example 2.11. The system

$$
u_{t}=v v_{x}, \quad v_{t}=v_{x x x}+2 u v_{x}+v u_{x}
$$

corresponds to

$$
L=D^{3}+2 u D+u_{x}+v D^{-1} v, \quad A=L_{+} .
$$

Example 2.12. The operators

$$
L=\left(D^{4}+u D^{2}+D^{2} u+v\right) D^{-1}, \quad A=L_{+}
$$

yield

$$
u_{t}=w_{x}, \quad w_{t}=w_{x x x}+w u_{x}+u w_{x},
$$

where $w=v+\alpha v_{x x}$ for some constant $\alpha$.

Example 2.13. For

$$
L=\left(D^{5}+u D^{3}+D^{3} u+v D+D v\right) D^{-1}, \quad A=\left(L^{\frac{3}{4}}\right)_{+}
$$

we obtain

$$
u_{t}=-u_{x x x}+w_{x}-u u_{x}, \quad w_{t}=2 w_{x x x}+u w_{x},
$$

where $w=v+\beta u_{x x}$ for some constant $\beta$.

Example 2.14. The operators

$$
L=\left(D^{3}+2 u D+u_{x} u+v D^{-1} v\right) D, \quad A=\left(L^{\frac{3}{4}}\right)_{+}
$$

correspond to

$$
u_{t}=u_{x x x}+u u_{x}-v v_{x}, \quad v_{t}=-2 v_{x x x}-u v_{x} .
$$

Several more examples can be found in $[12,13]$. 


\subsection{Matrix Lax pairs}

\subsubsection{The NLS hierarchy}

The nonlinear Schrödinger equation (NLS equation) has the form $Z_{t}=$ $i Z_{x x}+|Z|^{2} Z$. After a (complex) scaling of $t$ and $Z$ the equation can be written as a system of two equations

$$
u_{t}=\frac{1}{2}\left(u_{x x}-2 u^{2} v\right), \quad v_{t}=\frac{1}{2}\left(-v_{x x}+2 v^{2} u\right)
$$

where $u=Z, v=\bar{Z}$. The Lax representation (1.4) for (2.25) is defined [88] by

$$
\begin{gathered}
L=D+\left(\begin{array}{cc}
1 & 0 \\
0 & -1
\end{array}\right) \lambda+\left(\begin{array}{cc}
0 & u \\
v & 0
\end{array}\right), \\
A=\left(\begin{array}{cc}
1 & 0 \\
0 & -1
\end{array}\right) \lambda^{2}+\left(\begin{array}{cc}
0 & u \\
v & 0
\end{array}\right) \lambda+\frac{1}{2}\left(\begin{array}{cc}
-u v & -u_{x} \\
v_{x} & u v
\end{array}\right) .
\end{gathered}
$$

Notice that all matrix coefficients of $L$ and $A$ belong to the Lie algebra $\mathfrak{s l}_{2}$.

One can verify that the matrix $A$ obeys the following properties:

a) The commutator $[A, L]$ does not depend on $\lambda$;

b) It has the following matrix structure:

$$
[A, L]=\left(\begin{array}{ll}
0 & * \\
* & 0
\end{array}\right)
$$

It is clear that if these properties hold for a matrix polynomial

$$
A_{n}=\sum_{i=0}^{n} a_{i} \lambda^{i}, \quad a_{i} \in \mathfrak{s l}_{2}
$$

then the Lax equation $L_{t}=\left[A_{n}, L\right]$ is equivalent to a system of two evolution equations for $u$ and $v$.

Problem 2.2. How to describe all matrix polynomials (2.26) that satisfy the above two properties?

\section{Formal diagonalization}

Theorem 2.5. [12, Section 1]. There exists a unique series

$$
T=\mathbf{1}+\left(\begin{array}{cc}
0 & \alpha_{1} \\
\beta_{1} & 0
\end{array}\right) \frac{1}{\lambda}+\left(\begin{array}{cc}
0 & \alpha_{2} \\
\beta_{2} & 0
\end{array}\right) \frac{1}{\lambda^{2}}+\cdots
$$


such that

$$
T^{-1} L T=L_{0}
$$

where

$$
\begin{aligned}
L_{0}=D & +\left(\begin{array}{cc}
1 & 0 \\
0 & -1
\end{array}\right) \lambda+\left(\begin{array}{cc}
\rho_{0} & 0 \\
0 & -\rho_{0}
\end{array}\right)+\left(\begin{array}{cc}
\rho_{1} & 0 \\
0 & -\rho_{1}
\end{array}\right) \frac{1}{\lambda} \\
& +\left(\begin{array}{cc}
\rho_{2} & 0 \\
0 & -\rho_{2}
\end{array}\right) \frac{1}{\lambda^{2}}+\cdots
\end{aligned}
$$

Proof. Equating the coefficients of $\lambda^{0}$ in $L T=T L_{0}$, we get

$$
\left[\left(\begin{array}{cc}
1 & 0 \\
0 & -1
\end{array}\right),\left(\begin{array}{cc}
0 & \alpha_{1} \\
\beta_{1} & 0
\end{array}\right)\right]-\left(\begin{array}{cc}
\rho_{0} & 0 \\
0 & -\rho_{0}
\end{array}\right)=\left(\begin{array}{ll}
0 & u \\
v & 0
\end{array}\right) .
$$

Hence $\rho_{0}=0, \alpha_{1}=\frac{1}{2} u$ and $\beta_{1}=-\frac{1}{2} v$. At each step we have a similar relation of the form

$$
\left[\left(\begin{array}{cc}
1 & 0 \\
0 & -1
\end{array}\right),\left(\begin{array}{cc}
0 & \alpha_{k} \\
\beta_{k} & 0
\end{array}\right)\right]-\left(\begin{array}{cc}
\rho_{k-1} & 0 \\
0 & -\rho_{k-1}
\end{array}\right)=P_{k},
$$

where $P_{k} \in \mathfrak{s l}_{2}$ is a already known matrix. The functions $\alpha_{k}, \beta_{k}, \rho_{k-1}$ are thus uniquely defined.

Proposition 2.3. Let

$$
\begin{aligned}
& B_{n}=T\left(\begin{array}{cc}
1 & 0 \\
0 & -1
\end{array}\right) T^{-1} \lambda^{n}, \\
& A_{n}=\left(B_{n}\right)_{+}
\end{aligned}
$$

where, by definition,

$$
\left(\sum_{i=-\infty}^{m} a_{i} \lambda^{i}\right)_{+}^{\stackrel{\text { def }}{=}} \sum_{i=0}^{m} a_{i} \lambda^{i}, \quad\left(\sum_{i=-\infty}^{m} a_{i} \lambda^{i}\right)_{-}^{\stackrel{\text { def }}{=}} \sum_{i=-\infty}^{-1} a_{i} \lambda^{i} .
$$

Then $A_{n}$ satisfies properties a) and $b$ ).

Proof. Since $\left[L, B_{n}\right]=0$, we have

$$
\left[A_{n}, L\right]=-\left[\left(B_{n}\right)_{-}, L\right]
$$

The left hand side is a polynomial in $\lambda$ whereas the right hand side has the form

$$
\left(\begin{array}{ll}
0 & * \\
* & 0
\end{array}\right)+\sum_{i=-\infty}^{-1} b_{i} \lambda^{i}
$$

Hence

$$
\left[A_{n}, L\right]=\left(\begin{array}{cc}
0 & f_{n} \\
g_{n} & 0
\end{array}\right)
$$


Proposition 2.4. For any $n$ and $m$ the system of equations

$$
u_{\tau}=f_{m}, \quad v_{\tau}=g_{m}
$$

where $f_{i}$ and $g_{i}$ are defined by (2.29), is a higher symmetry for the system $u_{t}=f_{n}, \quad v_{t}=g_{n}$.

The proof is similar to the proof of Theorem 2.2 for the KdV hierarchy. Exercise 2.4. Prove the proposition.

The $A$-operator for the NLS equation is given by (2.28) with $n=2$. Formulas $(2.27),(2.28)$ for arbitrary $n$ define the NLS hierarchy. The next member of the NLS hierarchy

$$
u_{t}=-\frac{1}{4} u_{x x x}+\frac{3}{2} v u u_{x}, \quad v_{t}=-\frac{1}{4} v_{x x x}+\frac{3}{2} u v v_{x}
$$

corresponds to

$$
A_{3}=A_{2} \lambda+\frac{1}{4}\left(\begin{array}{cc}
v u_{x}-u v_{x} & u_{x x}-2 u^{2} v \\
v_{x x}-2 v^{2} u & u v_{x}-v u_{x}
\end{array}\right) .
$$

The reduction $v=u$ leads to the modified Korteweg-de Vries equation

$$
u_{t}=-\frac{1}{4} u_{x x x}+\frac{3}{2} u^{2} u_{x}
$$

\section{Recursion operator for NLS equation}

In this section we follow the paper [35, Section $3 \mathrm{~A}]$. Since

$$
B_{n+1}=\lambda B_{n}
$$

we have

$$
A_{n+1}=\left(\lambda B_{n}\right)_{+}=\lambda\left(B_{n}\right)_{+}+\left(\lambda\left(B_{n}\right)_{-}\right)_{+} .
$$

The latter formula shows that

$$
A_{n+1}=\lambda A_{n}+R_{n}
$$

where $R_{n}$ does not depend on $\lambda$. Substituting this into the Lax equation $L_{t_{n+1}}=\left[A_{n+1}, L\right]$, we get

$$
L_{t_{n+1}}=\lambda L_{t_{n}}+\left[R_{n}, L\right]
$$

If

$$
R_{n}=\left(\begin{array}{cc}
a_{n} & b_{n} \\
c_{n} & -a_{n}
\end{array}\right)
$$


then we find that

$$
b_{n}=\frac{1}{2} f_{n}, \quad c_{n}=-\frac{1}{2} g_{n}, \quad a_{n}=\frac{1}{2} D^{-1}\left(v f_{n}+u g_{n}\right) .
$$

Therefore the recursion operator

$$
\mathcal{R}\left(\begin{array}{c}
f_{n} \\
g_{n}
\end{array}\right)=\left(\begin{array}{c}
f_{n+1} \\
g_{n+1}
\end{array}\right), \quad\left(\begin{array}{c}
f_{1} \\
g_{1}
\end{array}\right)=\left(\begin{array}{c}
u_{x} \\
v_{x}
\end{array}\right)
$$

is given by

$$
\mathcal{R}=\left(\begin{array}{cc}
-\frac{1}{2} D+u D^{-1} v & u D^{-1} u \\
-v D^{-1} v & \frac{1}{2} D-v D^{-1} u
\end{array}\right)
$$

The operator $\mathcal{R}^{2}$ gives rise to a recursion operator for the $\mathrm{mKdV}$ equation by the reduction $v=u$.

\subsubsection{Generalizations}

Consider the operator

$$
L=D+\lambda a+q(x, t)
$$

where $q$ and $a$ belong to a Lie algebra $\mathcal{G}$ and $\lambda$ is the spectral parameter. The constant element $a$ is supposed to be such that

$$
\mathcal{G}=\operatorname{Ker}\left(a d_{a}\right) \oplus \operatorname{Im}\left(a d_{a}\right)
$$

Theorem 2.6. There exist unique series

$$
\begin{aligned}
& u=u_{-1} \lambda^{-1}+u_{-2} \lambda^{-2}+\cdots, \quad u_{i} \in \operatorname{Im}\left(a d_{a}\right), \\
& h=h_{0}+h_{-1} \lambda^{-1}+h_{-2} \lambda^{-2}+\cdots, \quad h_{i} \in \operatorname{Ker}\left(a d_{a}\right),
\end{aligned}
$$

such that

$$
e^{a d_{u}}(L) \stackrel{\text { def }}{=} L+[u, L]+\frac{1}{2}[u,[u, L]]+\cdots=D_{x}+a \lambda+h .
$$

Let $b$ be a constant element of $\mathcal{G}$ such that

$$
\left[b, \operatorname{Ker}\left(a d_{a}\right)\right]=\{0\} .
$$

Since

$$
\left[b \lambda^{n}, D_{x}+a \lambda+h\right]=0,
$$

we have $\left[B_{b, n}, L\right]=0$, where

$$
B_{b, n}=e^{-a d_{u}}\left(b \lambda^{n}\right) .
$$


Then the corresponding $A$-operator of the form

$$
A_{b, n}=b \lambda^{n}+a_{n-1} \lambda^{n-1}+\cdots+a_{0}
$$

is defined by the formula

$$
A_{b, n}=\left(B_{b, n}\right)_{+} .
$$

For the Lie algebra $\mathcal{G}=\mathfrak{s l}_{2}$ and $a=\operatorname{diag}(1,-1)$ we get the NLS hierarchy.

Example 2.15. Let $\mathcal{G}=\mathfrak{g l}_{m}$, the $L$-operator has the form (2.30), where

$$
a=\left(\begin{array}{cc}
\mathbf{1}_{m-1} & 0 \\
0 & -1
\end{array}\right), \quad q=\left(\begin{array}{cc}
0 & \mathbf{u} \\
\mathbf{v}^{t} & 0
\end{array}\right) .
$$

Here $\mathbf{u}$ and $\mathbf{v}$ are column vectors. In this case

$$
\operatorname{Ker}\left(a d_{a}\right)=\left\{\left(\begin{array}{cc}
\mathbf{S} & 0 \\
0 & s
\end{array}\right)\right\}, \quad \operatorname{Im}\left(a d_{a}\right)=\left\{\left(\begin{array}{cc}
0 & \mathbf{u}_{1} \\
\mathbf{u}_{2}^{t} & 0
\end{array}\right)\right\},
$$

where $\mathbf{S}$ is an $(m-1) \times(m-1)$-matrix, $s$ is a scalar and $\mathbf{u}_{i}$ are column vectors. Following the above diagonalization procedure, we find that the coefficients of the operator

$$
A_{a, 2}=a \lambda^{2}+s_{1} \lambda+s_{2}
$$

are given by

$$
s_{1}=\left(\begin{array}{cc}
0 & \mathbf{u} \\
\mathbf{v}^{t} & 0
\end{array}\right), \quad s_{2}=\frac{1}{2}\left(\begin{array}{cc}
-\mathbf{u v}^{t} & -\mathbf{u}_{x} \\
\mathbf{v}_{x}^{t} & \langle\mathbf{u}, \mathbf{v}\rangle
\end{array}\right) .
$$

If $m=2$ the Lax pair coincides with the Lax pair for the NLS equation from Section 2.2.1. The corresponding non-linear integrable system is (up to scalings of $t, \mathbf{u}, \mathbf{v})$ the vector NLS equation [47]

$$
\mathbf{u}_{t}=\mathbf{u}_{x x}+2\langle\mathbf{u}, \mathbf{v}\rangle \mathbf{u}, \quad \mathbf{v}_{t}=-\mathbf{v}_{x x}-2\langle\mathbf{v}, \mathbf{u}\rangle \mathbf{v} .
$$

Example 2.16. Let $\mathcal{G}=\mathfrak{g l}_{m}, a=\operatorname{diag}\left(a_{1}, \ldots, a_{m}\right), b=\operatorname{diag}\left(b_{1}, \ldots, b_{m}\right)$, where $a_{i} \neq a_{j}$ for $i \neq j$. The equation corresponding to $A_{1}$ given by $(2.31)$ is called $m$-wave equation. It has the form

$$
\mathbf{Q}_{t}=\mathbf{P}_{x}+[\mathbf{Q}, \mathbf{P}]
$$

where $\mathbf{Q}$ and $\mathbf{P}$ are $m \times m$-matrices whose entries are related by

$$
p_{i j}=\frac{b_{i}-b_{j}}{a_{j}-a_{i}} q_{i j} .
$$

Solutions of (2.33) that do not depend on $x$ describe the dynamics of an $m$-dimensional rigid body [46]. 


\section{Relations between scalar to matrix Lax pairs}

The Gelfand-Dikii hierarchy (see Section 2.1.3) is defined by a scalar linear differential operator of order $n$. Of course, it is not difficult to replace this operator by a matrix first order differential operator of the form

$$
\mathcal{L}=D+\Lambda+q
$$

where

$$
\Lambda=\left(\begin{array}{ccccc}
0 & & \cdots & 0 & \lambda \\
1 & & & & 0 \\
0 & \ddots & \ddots & & \\
\vdots & \ddots & \ddots & & \vdots \\
0 & \cdots & 0 & 1 & 0
\end{array}\right), \quad q=\left(\begin{array}{cccc}
0 & 0 & \cdots & u_{1} \\
0 & 0 & \cdots & u_{2} \\
0 & 0 & \cdots & u_{3} \\
\vdots & \vdots & & \vdots \\
0 & 0 & \cdots & u_{n}
\end{array}\right)
$$

Let us consider operators (2.34), where $q$ is an arbitrary upper-diagonal matrix. Any gauge transformation $\overline{\mathcal{L}}=N \mathcal{L} N^{-1}$, where $N$ is a function with values in the group of upper triangular matrices with ones on the diagonal, preserves the class of such operators. It turns out that the matrix $q$ defined by (2.35) is one of the possible canonical forms with respect to this gauge action.

The approach [12, Section 6] based on this observation allows one to construct an analog of the Gelfand-Dikii hierarchy for any Kac-Moody algebra $G$.

Let $e_{i}, f_{i}, h_{i}$, where $i=0, \ldots, r$, be the canonical generators of a KacMoody algebra $G$ with the commutator relations

$$
\left[h_{i}, h_{j}\right]=0, \quad\left[e_{i}, f_{j}\right]=\delta_{i j} h_{i}, \quad\left[h_{i}, e_{j}\right]=A_{i j} e_{j}, \quad\left[h_{i}, f_{j}\right]=-A_{i j} f_{j}
$$

where $A$ is the Cartan matrix of the algebra $G$.

Let us take $\sum_{i=0}^{r} e_{i}$ for the element $\Lambda$ in (2.34). The potential $q$ depends on a choice of a vertex $c_{m}$ for the Dynkin diagram of $G$. We consider the gradation $G=\oplus G_{i}$ such that $e_{m} \in G_{1}, f_{m} \in G_{-1}$ and the remaining canonical generators belong to $G_{0}$. It is well-known that $\mathcal{G}=G_{0}$ is a semisimple finite-dimensional Lie algebra. The potential $q$ is a generic element of the Borel subalgebra $\mathcal{B} \subset \mathcal{G}$ generated by $f_{i}, h_{i}$, where $i \neq m$.

If $\mathcal{L}$ is an operator of the form (2.34) and $S$ belongs to the corresponding nilpotent subalgebra $\mathcal{N} \subset \mathcal{B}$, then the operator

$$
\overline{\mathcal{L}}=e^{a d S}(\mathcal{L})
$$

has the same form (2.34) (with different $q$ ). This follows from the fact that $[\mathcal{N}, \mathcal{B}] \subset \mathcal{N}, \quad\left[\mathcal{N}, e_{m}\right]=\{0\}, \quad\left[\mathcal{N}, e_{i}\right] \subset \mathcal{B}$.

Any canonical form under these gauge transformations gives rise to a system of $r$ evolution equations. The systems corresponding to different 
canonical forms are related by invertible polynomial transformations of unknown functions.

Moreover, any $\mathcal{L}$-operator (2.34) generates a commutative hierarchy of integrable systems. The corresponding $A$-operators can be constructed by a formal diagonalization procedure, which generalizes the construction from Theorem 2.6.

It was proved in [12] that the systems related to $L$-operators of the form (2.34) include the systems from Theorem 2.4.

For further generalizations see $[10,31,11]$.

\subsection{Decomposition of loop algeras and Lax pairs}

In all classes of Lax representations described above, $L$-operators are polynomials in the spectral parameter $\lambda$. However, there exist important examples, where $\lambda$ is a parameter on an elliptic curve or on its degenerations $[69,87]$.

An algebraic curve of genus $g>1$ appears in the following Example 2.17. [32] Consider the vector equation

$$
\mathbf{u}_{t}=\left(\mathbf{u}_{x x}+\frac{3}{2}\left\langle\mathbf{u}_{x}, \mathbf{u}_{x}\right\rangle \mathbf{u}\right)_{x}+\frac{3}{2}\langle\mathbf{u}, \mathbf{R} \mathbf{u}\rangle \mathbf{u}_{x}, \quad|\mathbf{u}|=1,
$$

where $\mathbf{u}=\left(u^{1}, \ldots, u^{N}\right), \mathbf{R}=\operatorname{diag}\left(r_{1}, \ldots, r_{N}\right)$, and $\langle\cdot, \cdot\rangle$ is the standard scalar product. In the case $N=3$ this equation is a higher symmetry of the famous integrable Landau-Lifshitz equation.

$$
\mathbf{u}_{t}=\mathbf{u} \times \mathbf{u}_{x x}+\mathbf{R} \mathbf{u} \times \mathbf{u}, \quad|\mathbf{u}|=1 .
$$

Here $\times$ stands for the cross product. It is interesting that for $N \neq 3$ all symmetries of equation (2.36) have odd orders. In particular, the equation has no symmetry of order 2.

Equation (2.36) possesses a Lax representation with

$$
L=D+\left(\begin{array}{cc}
0 & \Lambda \mathbf{u} \\
\mathbf{u}^{T} \Lambda & 0
\end{array}\right)
$$

Here

$$
\Lambda=\operatorname{diag}\left(\lambda_{1}, \lambda_{2}, \cdots, \lambda_{N}\right)
$$

is a matrix defined by

$$
\Lambda^{2}=\frac{1}{\lambda^{2}}-\mathbf{R}
$$

It is clear that

$$
\lambda_{1}^{2}+r_{1}=\lambda_{2}^{2}+r_{2}=\cdots=\lambda_{N}^{2}+r_{N} .
$$


For generic $\lambda_{i}, r_{i}$ this algebraic curve has genus $g=1+(N-3) 2^{N-2}$. In the case $N=3$ such a form of the elliptic spectral curve has been used in $[69,18]$.

A class of Lax operators related to algebraic curves of genus $g>1$ was introduced in $[40,42]$.

\section{Factoring subalgebras}

If we don't want to fix a priori the $\lambda$-dependence in Lax operators, we may assume that $L$ is a Laurent series in $\lambda$ with coefficients being elements of a finite-dimensional Lie algebra $\mathcal{G}$.

The Lie algebra $\mathcal{G}((\lambda))$ of formal series of the form

$$
\mathcal{G}((\lambda))=\left\{\sum_{i=-n}^{\infty} g_{i} \lambda^{i} \quad \mid \quad g_{i} \in \mathcal{G}, \quad n \in \mathbb{Z}\right\}
$$

is called the (extended) loop algebra over $\mathcal{G}$.

If $\mathcal{G}$ is semi-simple, then the formula

$$
\langle X(\lambda), Y(\lambda)\rangle=\operatorname{res}(X(\lambda), Y(\lambda)), \quad X(\lambda), Y(\lambda) \in \mathcal{G}((\lambda))
$$

defines an invariant non-degenerate bi-linear form on $\mathcal{G}((\lambda))$. Here $(\cdot, \cdot)$ is the non-degenerate invariant Killing form on $\mathcal{G}$, res $P$ stands for the coefficient of $\lambda^{-1}$ in a (scalar) Laurent series $P$. The invariance of the form means that

$$
\langle[a, b], c\rangle=-\langle b,[a, c]\rangle
$$

for any $a, b, c \in \mathcal{G}((\lambda))$.

If we assume that $L$ and $A$ in Lax equation (1.4) are elements of $\mathcal{G}((\lambda))$, then (1.4) is equivalent to an infinite set of evolution equations. To get a finite system of PDEs we need some additional assumptions on the structure of $L$ and $A$.

The basic ingredient for constructing of Lax pairs in $\mathcal{G}((\lambda))$ is a vector space decomposition (see $[7,70]$ )

$$
\mathcal{G}((\lambda))=\mathcal{G}[[\lambda]] \oplus \mathcal{U}
$$

where $\mathcal{G}[[\lambda]]$ is the subalgebra of all Taylor series and $\mathcal{U}$ is a so called factoring, or complementary, Lie subalgebra. Obviously, the subalgebra $\mathcal{G}[[\lambda]]$ is isotropic with respect to the form (2.39).

Let us denote by $\pi_{+}$and $\pi_{-}$the projection operators onto $\mathcal{U}$ and $\mathcal{G}[[\lambda]]$, respectively.

The following statement is evident:

Lemma 2.4. Let $\mathcal{U}$ be a factoring subalgera. Than for any principle part $P=\sum_{i=-n}^{-1} g_{i} \lambda^{i}$, where $g_{i} \in \mathcal{G}$, there exists a unique element $\bar{P} \in \mathcal{U}$ of the form $\bar{P}=P+O(1)$. 
Example 2.18. The simplest factoring subalgebra is given by

$$
\mathcal{U}^{s t}=\left\{\sum_{i=1}^{n} g_{i} \lambda^{-i} \quad \mid \quad g_{i} \in \mathcal{G}, \quad n \in \mathbb{N}\right\} .
$$

This subalgebra is called standard.

Two factoring subalgebras are called equivalent if they are related by a transformation of the parameter $\lambda$ of the form

$$
\lambda \rightarrow \lambda+k_{2} \lambda^{2}+k_{3} \lambda^{3}+\cdots, \quad k_{i} \in \mathbb{C},
$$

or by an automorphism of the form

$$
\exp \left(\operatorname{ad}_{g_{1} \lambda+g_{2} \lambda^{2}+\cdots}\right), \quad g_{i} \in \mathcal{G} .
$$

It is clear the (2.42) and (2.43) preserve the subalgebra $\mathcal{G}[[\lambda]]$.

Suppose that $r$-dimensional Lie algebra $\mathcal{G}$ is semi-simple. Let $\mathbf{e}_{1}, \ldots, \mathbf{e}_{r}$ be a basis in $\mathcal{G}$. According Lemma 2.4 for any $i$ there exists a unique element $\mathbf{E}_{i} \in \mathcal{U}$ such that

$$
\mathbf{E}_{i}=\frac{\mathbf{e}_{i}}{\lambda}+O(1)
$$

Proposition 2.5. The elements $\mathbf{E}_{i}$ generate $\mathcal{U}$.

Proof. We have to show that for any $i, k$ an element $\mathbf{E}_{i k}$ of the form

$$
\mathbf{E}_{i k}=\frac{\mathbf{e}_{i}}{\lambda^{k}}+O(-k+1)
$$

can be obtained as a commutator of length $k$ of the elements $\mathbf{E}_{j}$, where $j=1, \ldots, r$. The proof is by induction on $k$. The induction step follows from the well-known property of the semi-simple Lie algebras: $[\mathcal{G}, \mathcal{G}]=\mathcal{G}$.

If we take generic elements of the form (2.44), the Lie subalgebra they generate will contain Taylor series. All of them should be equal to zero. This imposes strong restrictions on generators (2.44).

Lemma 2.5. A subalgebra $\mathcal{U}$ is factoring iff for any $k$ the dimension $d_{k}$ of the vector space $V_{k}$ of all elements from $\mathcal{U}$ of the form

$$
\sum_{i=-n}^{\infty} g_{i} \lambda^{i}, \quad n \leq k
$$

is the same as for the standard subalgebra (2.41). 
Proof. Let $\mathbf{F}_{i j}$, where $i \leq r, j \leq k$, be elements of $\mathcal{U}$ such that

$$
\mathbf{F}_{i j}=\frac{\mathbf{e}_{i}}{\lambda^{j}}+O(1)
$$

It is clear that $\mathbf{F}_{i j}$ form a basis of $V_{k}$.

Conjecture 2.1. Let $\mathcal{G}$ be a simple Lie algebra not isomorphic to $\mathfrak{s l}_{2}$. Then elements $\mathbf{E}_{i} \in \mathcal{G}((\lambda))$ of the form (2.44) generate a factoring subalgebra iff the dimension of the vector space spanned by $\left[\mathbf{E}_{j}, \mathbf{E}_{k}\right]$ and $\mathbf{E}_{i}$ is equal to $2 \operatorname{dim} \mathcal{G}$.

When the factoring subalgebra $\mathcal{U}$ is isotropic with respect to $(2.39)$, the description of factoring subalgebras is closely related to a classification of the Yang-Baxter $r$-matrices [5]. Without this assumption the problem has not been deeply considered yet. In Subsection 2.3 .1 we solve it for $\mathcal{G}=\mathfrak{s o}_{3}$ [76].

\section{Multiplicands}

Definition 2.4. A (scalar) Laurent series

$$
\mathbf{m}=\sum_{i=-n}^{\infty} c_{i} \lambda^{i}, \quad c_{i} \in \mathbb{C},
$$

is called a multiplicand of $\mathcal{U}$ if $\mathbf{m} \mathcal{U} \subset \mathcal{U}$. The number $n$ is called the order of the multiplicand $\mathbf{m}$. If $\mathcal{U}$ admits a multiplicand $\mathbf{m}$ of order $n=1$, then $\mathcal{U}$ is called homogeneous.

Let $\mathcal{G}$ be a simple Lie algebra. The following construction allows us to associate an algebraic curve with any factoring subalgebra $\mathcal{U}$.

Theorem 2.7. [62] For any factoring subalgebra the following statements are fulfilled:

i) multiplicands of negative orders do not exist;

ii) the complement of the set of all multiplicand orders with respect to the set of natural numbers is finite.

It follows from the statement ii) that any two multiplicands are related by an algebraic relation. So, the set of all multiplicands is isomorphic to a coordinate ring of some algebraic curve. Examples are given in Section 2.3 .1 .

This canonical relation between factoring subalgebras and algebraic curves allows one to use methods of algebraic geometry for the investigation of factoring subalgebras. 


\subsubsection{Factoring subalgebras for $\mathcal{G}=\mathfrak{s o}_{3}$}

In this section we follow the paper [76].

Consider the standard basis

$$
\mathbf{e}_{1}=\left(\begin{array}{ccc}
0 & 1 & 0 \\
-1 & 0 & 0 \\
0 & 0 & 0
\end{array}\right), \quad \mathbf{e}_{2}=\left(\begin{array}{ccc}
0 & 0 & 1 \\
0 & 0 & 0 \\
-1 & 0 & 0
\end{array}\right), \quad \mathbf{e}_{3}=\left(\begin{array}{ccc}
0 & 0 & 0 \\
0 & 0 & 1 \\
0 & -1 & 0
\end{array}\right)
$$

in $\mathfrak{s o}_{3}$. Let $\mathcal{U}$ be a factoring subalgebra. Define elements $\mathbf{E}_{i} \in \mathcal{U}$ by (2.44).

Automorphisms (2.43) are orthogonal transformations, which are Taylor series in $\lambda$. The functions

$$
\left|\mathbf{E}_{1}\right|^{2}, \quad\left|\mathbf{E}_{2}\right|^{2}, \quad\left|\mathbf{E}_{3}\right|^{2}, \quad\left(\mathbf{E}_{1}, \mathbf{E}_{2}\right), \quad\left(\mathbf{E}_{1}, \mathbf{E}_{3}\right), \quad\left(\mathbf{E}_{2}, \mathbf{E}_{3}\right),
$$

where

$$
\left(\sum_{i} x_{i} \mathbf{e}_{\mathbf{i}}, \sum_{j} y_{j} \mathbf{e}_{\mathbf{j}}\right)=\sum_{i} x_{i} y_{i}
$$

are invariants for the transformations (2.43).

Proposition 2.6. For any factoring subalgebra the following relations hold:

$$
\left(\begin{array}{c}
{\left[\mathbf{E}_{1},\left[\mathbf{E}_{2}, \mathbf{E}_{3}\right]\right]} \\
{\left[\mathbf{E}_{3},\left[\mathbf{E}_{1}, \mathbf{E}_{2}\right]\right]} \\
{\left[\mathbf{E}_{2},\left[\mathbf{E}_{3}, \mathbf{E}_{1}\right]\right]}
\end{array}\right)=\mathbf{A}\left(\begin{array}{c}
{\left[\mathbf{E}_{3}, \mathbf{E}_{1}\right]} \\
{\left[\mathbf{E}_{1}, \mathbf{E}_{2}\right]} \\
{\left[\mathbf{E}_{2}, \mathbf{E}_{3}\right]}
\end{array}\right)+\mathbf{B}\left(\begin{array}{c}
\mathbf{E}_{2} \\
\mathbf{E}_{3} \\
\mathbf{E}_{1}
\end{array}\right)
$$

$$
\left(\begin{array}{c}
{\left[\mathbf{E}_{3},\left[\mathbf{E}_{2}, \mathbf{E}_{3}\right]\right]+\left[\mathbf{E}_{1},\left[\mathbf{E}_{1}, \mathbf{E}_{2}\right]\right]} \\
{\left[\mathbf{E}_{1},\left[\mathbf{E}_{3}, \mathbf{E}_{1}\right]\right]+\left[\mathbf{E}_{2},\left[\mathbf{E}_{2}, \mathbf{E}_{3}\right]\right]} \\
{\left[\mathbf{E}_{2},\left[\mathbf{E}_{1}, \mathbf{E}_{2}\right]\right]+\left[\mathbf{E}_{3},\left[\mathbf{E}_{3}, \mathbf{E}_{1}\right]\right]}
\end{array}\right)=\mathbf{C}\left(\begin{array}{c}
{\left[\mathbf{E}_{3}, \mathbf{E}_{1}\right]} \\
{\left[\mathbf{E}_{1}, \mathbf{E}_{2}\right]} \\
{\left[\mathbf{E}_{2}, \mathbf{E}_{3}\right]}
\end{array}\right)+\mathbf{D}\left(\begin{array}{c}
\mathbf{E}_{2} \\
\mathbf{E}_{3} \\
\mathbf{E}_{1}
\end{array}\right)
$$

where

$$
\begin{aligned}
\mathbf{A}=\left(\begin{array}{ccc}
-u & w & 0 \\
u & 0 & -v \\
0 & -w & v
\end{array}\right), & \mathbf{B}=\left(\begin{array}{ccc}
-\alpha & \beta & 0 \\
\alpha & 0 & -\gamma \\
0 & -\beta & \gamma
\end{array}\right), \\
\mathbf{C}=\left(\begin{array}{ccc}
x & v & -w \\
-v & y & u \\
w & -u & z
\end{array}\right), & \mathbf{D}=\left(\begin{array}{ccc}
\varepsilon & \gamma & -\beta \\
-\gamma & \tau & \alpha \\
\beta & -\alpha & \delta
\end{array}\right)
\end{aligned}
$$

are constant matrices. Moreover $\operatorname{tr} \mathbf{C}=\operatorname{tr} \mathbf{D}=0$ and

$$
c_{1} \mathbf{A}+c_{2} \mathbf{B}=0, \quad c_{1} \mathbf{C}+c_{2} \mathbf{D}=0
$$

for some constants $c_{1}, c_{2}$. 
Proof. The coefficients of $\lambda^{-3}$ in the expressions from the left hand side of (2.45) are equal to zero. Therefore, the expressions should be linear combinations of

$$
\mathbf{E}_{1}, \quad \mathbf{E}_{2}, \quad \mathbf{E}_{3}, \quad\left[\mathbf{E}_{1}, \mathbf{E}_{2}\right], \quad\left[\mathbf{E}_{3}, \mathbf{E}_{1}\right], \quad\left[\mathbf{E}_{2}, \mathbf{E}_{3}\right]
$$

The relations between the coefficients of these linear combinations follow from Lemma 2.4.

Remark 2.4. Proposition 2.6 means that $d_{3}=9$ (see Lemma 2.5).

Example 2.19. For the standard factoring subalgebra (2.41) conditions (2.45) are fulfilled with $\mathbf{A}=\mathbf{B}=\mathbf{C}=\mathbf{D}=0$. In this case,

$$
\mathbf{E}_{i}=\frac{\mathbf{e}_{i}}{\lambda}, \quad i=1,2,3
$$

It is clear that the algebra of multiplicands is generated by $x=\frac{1}{\lambda}$ and therefore the corresponding algebraic curve is a straight line.

Example 2.20. Suppose that

$$
\begin{aligned}
\left(\mathbf{E}_{3}, \mathbf{E}_{1}\right) & =-\alpha, \quad\left(\mathbf{E}_{1}, \mathbf{E}_{2}\right)=-\beta, \quad\left(\mathbf{E}_{2}, \mathbf{E}_{3}\right)=-\gamma, \\
\left|\mathbf{E}_{3}\right|^{2}-\left|\mathbf{E}_{1}\right|^{2}=\varepsilon, \quad\left|\mathbf{E}_{1}\right|^{2}-\left|\mathbf{E}_{2}\right|^{2} & =\tau, \quad\left|\mathbf{E}_{2}\right|^{2}-\left|\mathbf{E}_{3}\right|^{2}=\delta,
\end{aligned}
$$

where $\alpha, \beta, \gamma, \delta, \varepsilon, \tau$ are fixed constants such that $\varepsilon+\tau+\delta=0$. It follows from (2.48) that we may implement the spectral parameter $\lambda$ by formulas

$$
\left|\mathbf{E}_{1}\right|=\frac{\sqrt{1-p \lambda^{2}}}{\lambda}, \quad\left|\mathbf{E}_{2}\right|=\frac{\sqrt{1-q \lambda^{2}}}{\lambda}, \quad\left|\mathbf{E}_{3}\right|=\frac{\sqrt{1-r \lambda^{2}}}{\lambda}
$$

where $\varepsilon=p-r, \tau=q-p, \delta=r-q$. The elements $\mathbf{E}_{i}$ of the form

$\mathbf{E}_{1}=c_{1} \mathbf{e}_{1}, \quad \mathbf{E}_{2}=c_{2} \mathbf{e}_{1}+c_{3} \mathbf{e}_{2}, \quad \mathbf{E}_{3}=c_{4} \mathbf{e}_{1}+c_{5} \mathbf{e}_{2}+c_{6} \mathbf{e}_{3}, \quad c_{i} \in \mathbb{C}((\lambda))$,

can be easily reconstructed.

One can verify that such elements $\mathbf{E}_{i}$ satisfy (2.45), (2.46) with $\mathbf{A}=$ $\mathbf{C}=0$ and generate a factoring subalgebra. This factoring subalgebra is isotropic with respect to the form (2.39).

The expressions $X_{i}(\lambda)=\left|\mathbf{E}_{i}\right|$ are functions on the elliptic curve

$$
X_{1}^{2}+p=X_{2}^{2}+q=X_{3}^{2}+r .
$$

The functions

$$
x=\frac{1}{\lambda^{2}}, \quad y=\frac{\sqrt{\left(1-p \lambda^{2}\right)\left(1-q \lambda^{2}\right)\left(1-r \lambda^{2}\right)}}{\lambda^{3}}
$$


are multiplicands of $\mathcal{U}$ of order 2 and 3, respectively. For example, in the special case $\alpha=\beta=\gamma=0$ we have

$$
\begin{aligned}
& \mathbf{E}_{1}=\frac{\sqrt{1-p \lambda^{2}}}{\lambda} \mathbf{e}_{1}, \\
& \mathbf{E}_{2}=\frac{\sqrt{1-q \lambda^{2}}}{\lambda} \mathbf{e}_{2}, \\
& \mathbf{E}_{3}=\frac{\sqrt{1-r \lambda^{2}}}{\lambda} \mathbf{e}_{3},
\end{aligned}
$$

and

$$
x \mathbf{E}_{\mathbf{2}}=\left[\left[\mathbf{E}_{\mathbf{1}}, \mathbf{E}_{\mathbf{2}}\right], \mathbf{E}_{\mathbf{1}}\right]+p \mathbf{E}_{\mathbf{2}}, \quad y \mathbf{E}_{\mathbf{2}}=\left[\left[\mathbf{E}_{\mathbf{2}}, \mathbf{E}_{\mathbf{3}}\right],\left[\mathbf{E}_{\mathbf{1}}, \mathbf{E}_{\mathbf{2}}\right]\right]
$$

and so on. The corresponding algebraic curve is elliptic:

$$
y^{2}=(x-p)(x-q)(x-r) .
$$

Example 2.21. Let

$$
\begin{array}{ll}
\left|\mathbf{E}_{1}\right|^{2}=(\mu-r)(\mu-q)-u^{2}, & \left|\mathbf{E}_{2}\right|^{2}=(\mu-r)(\mu-p)-v^{2}, \\
\left|\mathbf{E}_{3}\right|^{2}=(\mu-q)(\mu-p)-w^{2}, & \left(\mathbf{E}_{1}, \mathbf{E}_{2}\right)=w(\mu-r)+u v, \\
\left(\mathbf{E}_{1}, \mathbf{E}_{3}\right)=v(\mu-q)+u w, & \left(\mathbf{E}_{2}, \mathbf{E}_{3}\right)=u(\mu-p)+v w,
\end{array}
$$

where $\mu=\lambda^{-1}, p, q, r, u, v, w$ are arbitrary parameters. The elements $\mathbf{E}_{i}, i=1,2,3$ satisfy (2.45), (2.46) with $x=p-r, y=q-p, z=r-q$, $\mathbf{B}=\mathbf{D}=0$ and generate a factoring subalgebra.

If $u=v=w=0$, then $\mathbf{E}_{i}$ are given by

$$
\begin{aligned}
& \mathbf{E}_{\mathbf{1}}=\frac{\sqrt{(1-r \lambda)(1-q \lambda)}}{\lambda} \mathbf{e}_{\mathbf{1}}, \\
& \mathbf{E}_{\mathbf{2}}=\frac{\sqrt{(1-r \lambda)(1-p \lambda)}}{\lambda} \mathbf{e}_{\mathbf{2}}, \\
& \mathbf{E}_{\mathbf{3}}=\frac{\sqrt{(1-q \lambda)(1-p \lambda)}}{\lambda} \mathbf{e}_{\mathbf{3}} .
\end{aligned}
$$

At first glance, we deal with the functions

$$
X_{1}=\sqrt{\frac{(1-r \lambda)}{\lambda}}, \quad X_{2}=\sqrt{\frac{(1-q \lambda)}{\lambda}}, \quad X_{3}=\sqrt{\frac{(1-p \lambda)}{\lambda}}
$$

on the elliptic curve (2.49), but in fact $\mathbf{E}_{i}$ depend on the products

$$
Z_{1}=X_{2} X_{3}, \quad Z_{2}=X_{1} X_{3}, \quad Z_{3}=X_{1} X_{2}
$$


only. The corresponding algebraic curve can be written as

$$
\frac{Z_{1} Z_{2}}{Z_{3}}+p=\frac{Z_{1} Z_{3}}{Z_{2}}+q=\frac{Z_{2} Z_{3}}{Z_{1}}+r .
$$

This curve is rational. Indeed, substituting

$$
Z_{3}=\frac{(q-r) Z_{2} Z_{1}}{Z_{2}^{2}-Z_{1}^{2}}
$$

into the curve, we get

$$
\left(Z_{2}^{2}-Z_{1}^{2}\right)^{2}+a^{2} Z_{2}^{2}-b^{2} Z_{1}^{2}=0,
$$

where $a^{2}=(r-q)(q-p), \quad b^{2}=(r-p)(q-p)$. The latter curve admits the rational parameterization

$$
Z_{1}=\frac{a\left(t^{3}+S t\right)}{t^{4}+K t^{2}+S^{2}}, \quad Z_{2}=\frac{b\left(t^{3}-S t\right)}{t^{4}+K t^{2}+S^{2}}
$$

where

$$
S=\frac{\left(a^{2}-b^{2}\right)^{2}}{4 a^{2} b^{2}}, \quad K=\frac{a^{4}-b^{4}}{2 a^{2} b^{2}} .
$$

The algebra of multiplicands of the factoring subalgebra is generated by $x=\frac{1}{\lambda}$. For example,

$$
x \mathbf{E}_{1}=\left[\mathbf{E}_{3}, \mathbf{E}_{2}\right]+p \mathbf{E}_{1} .
$$

Example 2.22. Let

$$
\mathbf{E}_{i}=\frac{\mathbf{e}_{i}}{\lambda}+\nu\left[\mathbf{V}, \mathbf{e}_{i}\right]+\frac{1}{2}\left[\mathbf{V},\left[\mathbf{V}, \mathbf{e}_{i}\right]\right],
$$

where

$$
\mathbf{V}=v_{1} \mathbf{e}_{1}+v_{2} \mathbf{e}_{2}+v_{3} \mathbf{e}_{3},
$$

$\nu$ and $v_{i}$ are parameters. The constants in (2.45)- (2.47) are given by

$$
\begin{gathered}
u=v_{1} v_{3}, \quad v=v_{2} v_{3}, \quad w=v_{1} v_{2}, \quad x=v_{1}^{2}-v_{3}^{2}, \quad y=v_{2}^{2}-v_{1}^{2}, \quad z=v_{3}^{2}-v_{2}^{2}, \\
c_{1}=-1, \quad c_{2}=\nu^{2}+\frac{\Delta}{4}, \quad \Delta=v_{1}^{2}+v_{2}^{2}+v_{3}^{2} .
\end{gathered}
$$

The elements $\mathbf{E}_{i}$ generate a factoring subalgebra. The multiplicands of second and third order are given by

$$
x=\frac{1}{\lambda^{2}}-\frac{\Delta}{\lambda}, \quad y=\frac{1}{\lambda^{3}}+\frac{\Delta\left(4 \nu^{2}-3 \Delta\right)}{4 \lambda} .
$$

Exercise 2.5. Verify that $x$ and $y$ are related by a degenerate elliptic curve with canonical form

$$
\bar{y}^{2}=4(\bar{x}-a)(\bar{x}-b)^{2},
$$

where

$$
a=\frac{2}{3} \nu^{2} \Delta, \quad b=-\frac{1}{3} \nu^{2} \Delta .
$$


Theorem 2.8. Any factoring subalgebra for $\mathcal{G}=\mathfrak{s o}_{3}$ is equivalent to one from Examples 2.19-2.22.

Proof. For a proof see [76].

A classification of factoring subalgebras for the semi-simple Lie algebra $\mathcal{G}=\mathfrak{s o}_{4}$ is important for applications. A class of factoring subalgebras was constructed in [15].

Open problem 2.1. Describe all factoring subalgebras for $\mathcal{G}=\mathfrak{s o}_{4}$.

\subsubsection{Integrable top-like systems}

As it was mentioned in Remark 1.1, one may assume that the $A$-operator in (1.4) belongs to $\mathcal{G}$ while $L$ belongs to a module over $\mathcal{G}$. In this section we assume that $\mathcal{G}$ is semi-simple.

For integrable top-like systems the $A$-operator in (1.4) belongs to $\mathcal{U}$ and $L$ belongs to the orthogonal complement $\mathcal{U}^{\perp}$ with respect to the scalar product (2.39). It follows from its invariance that $\mathcal{U}^{\perp}$ is a module over $\mathcal{U}$. Exercise 2.6. Prove that $\mathcal{U}^{\perp}$ does not contain non-zero Taylor series.

It was shown in [28, Theorem 2.3] that in this case natural Hamiltonian structures arise.

We say that an $L$-operator has order $k$ if $L \in \mathcal{O}_{k} \stackrel{\text { def }}{=} \lambda^{-k} \mathcal{G}[[\lambda]] \cap \mathcal{U}^{\perp}$. To construct $A$-operators we generalize the scheme of Sections 2.1.2, 2.2.1. Namely, we find elements of $\mathcal{G}((\lambda))$ that commute with $L$ and project them onto $\mathcal{U}$.

For the sake of simplicity we assume that $\mathcal{G}$ is embedded into a matrix algebra. Suppose that $B_{i j}=\lambda^{i} L^{j}$ belongs to $\mathcal{G}((\lambda))$.

Proposition 2.7. Suppose that $L \in \mathcal{O}_{k}$. Then

i) $\left[\pi_{+}\left(B_{i j}\right), L\right] \in \mathcal{O}_{k}$,

ii) for any $i, j, p, q$ the Lax equations

$$
L_{t}=\left[\pi_{+}\left(B_{i j}\right), L\right]
$$

and

$$
L_{\tau}=\left[\pi_{+}\left(B_{p q}\right), L\right]
$$

are infinitesimal symmetries for each other.

Here we denote by $\pi_{+}$the projection operator onto $\mathcal{U}$ parallel to $\mathcal{G}[[\lambda]]$.

Exercise 2.7. Prove the proposition (see proof of Theorem 2.2).

A general theory of Lax pairs of such kind and of the corresponding Hamiltonian structures for any semi-simple Lie algebra $\mathcal{G}$ was presented in [28]. Below we consider the case $\mathcal{G}=\mathfrak{s o}_{3}$. 


\subsection{3 $\mathfrak{s o}_{3}$ classical spinning tops}

In this section we demonstrate [28, Section 4] that the factoring subalgebras in $\mathfrak{s o}_{3}$ described in Section 2.3.1 are in one-to-one correspondence with classical integrable cases for the Kirchhoff problem of the motion of a rigid body in an ideal fluid [6]. The equations of motion are given by

$$
\frac{d \boldsymbol{\Gamma}}{d t}=\boldsymbol{\Gamma} \times \frac{\partial H}{\partial \mathbf{M}}, \quad \frac{d \mathbf{M}}{d t}=\mathbf{M} \times \frac{\partial H}{\partial \mathbf{M}}+\mathbf{\Gamma} \times \frac{\partial H}{\partial \boldsymbol{\Gamma}},
$$

where $\mathbf{M}=\left(M_{1}, M_{2}, M_{3}\right)$ is the total angular momentum, $\boldsymbol{\Gamma}=\left(\gamma_{1}, \gamma_{2}, \gamma_{3}\right)$ is the gravitational vector, $\times$ stands for the cross product,

$$
\frac{\partial H}{\partial \mathbf{M}}=\left(\frac{\partial H}{\partial M_{1}}, \frac{\partial H}{\partial M_{2}}, \frac{\partial H}{\partial M_{3}}\right), \quad \frac{\partial H}{\partial \boldsymbol{\Gamma}}=\left(\frac{\partial H}{\partial \gamma_{1}}, \frac{\partial H}{\partial \gamma_{2}}, \frac{\partial H}{\partial \gamma_{3}}\right)
$$

and the quadratic form $H(\mathbf{M}, \boldsymbol{\Gamma})$ is a Hamiltonian.

\section{Structure of the orthogonal complement to $\mathcal{U}$}

Proposition 2.8. The orthogonal complement to $\mathcal{U}$ can be described as follows:

i) There exist unique elements $\mathbf{R}_{i} \in \mathcal{U}^{\perp}$ of the form

$$
\mathbf{R}_{i}=\frac{\mathbf{e}_{i}}{\lambda}+O(1), \quad i=1,2,3
$$

They generate $\mathcal{U}^{\perp}$ as a $\mathcal{U}$-module.

ii) The following commutator relations hold:

$$
\begin{gathered}
\left(\begin{array}{c}
{\left[\mathbf{E}_{1}, \mathbf{R}_{1}\right]} \\
{\left[\mathbf{E}_{3}, \mathbf{R}_{3}\right]} \\
{\left[\mathbf{E}_{2}, \mathbf{R}_{2}\right]}
\end{array}\right)=\mathbf{A}\left(\begin{array}{c}
\mathbf{R}_{2} \\
\mathbf{R}_{3} \\
\mathbf{R}_{1}
\end{array}\right), \\
\left(\begin{array}{c}
{\left[\mathbf{E}_{3}, \mathbf{R}_{1}\right]+\left[\mathbf{E}_{1}, \mathbf{R}_{3}\right]} \\
{\left[\mathbf{E}_{1}, \mathbf{R}_{2}\right]+\left[\mathbf{E}_{2}, \mathbf{R}_{1}\right]} \\
{\left[\mathbf{E}_{2}, \mathbf{R}_{3}\right]+\left[\mathbf{E}_{3}, \mathbf{R}_{2}\right]}
\end{array}\right)=\mathbf{C}\left(\begin{array}{c}
\mathbf{R}_{2} \\
\mathbf{R}_{3} \\
\mathbf{R}_{1}
\end{array}\right),
\end{gathered}
$$

where $\mathbf{A}$ and $\mathbf{C}$ are matrices defined by (2.45), (2.46).

Remark 2.5. Elements $\mathbf{R}_{i} \in \mathfrak{s o}_{3}((\lambda))$ of the form (2.54) are determined by the commutator relations up to a summand of the form $S(\lambda) \mathbf{e}_{i}$, where $S$ is a scalar Taylor series. 
Open problem 2.2. Prove that for any $S(\lambda)$ the $\mathcal{U}$-module generated by $\mathbf{R}_{i}, i=1,2,3$ is the orthogonal complement to $\mathcal{U}$ with respect to the form

$$
\langle X(\lambda), Y(\lambda)\rangle_{P}=\operatorname{res} P(\lambda)(X(\lambda), Y(\lambda)), \quad X(\lambda), Y(\lambda) \in \mathfrak{s o}_{3}((\lambda))
$$

with a proper scalar Taylor series $P$.

The simplest option $L \in \mathcal{O}_{1}, \quad A=\pi_{+}(L)$ corresponds to integrable models of Euler type. In this case we have

$$
L=M_{1} \mathbf{R}_{1}+M_{2} \mathbf{R}_{\mathbf{2}}+M_{3} \mathbf{R}_{\mathbf{3}}, \quad A=M_{1} \mathbf{E}_{\mathbf{1}}+M_{2} \mathbf{E}_{\mathbf{2}}+M_{3} \mathbf{E}_{\mathbf{3}},
$$

The Lax pairs for integrable Kirchhoff type systems have the following form:

$$
\begin{aligned}
& L=\gamma_{1}\left[\mathbf{R}_{3}, \mathbf{E}_{2}\right]+\gamma_{2}\left[\mathbf{R}_{1}, \mathbf{E}_{3}\right]+\gamma_{3}\left[\mathbf{R}_{2}, \mathbf{E}_{1}\right]+m_{1} \mathbf{R}_{1}+m_{2} \mathbf{R}_{2}+m_{3} \mathbf{R}_{3} \\
& A=\pi_{+}(\lambda L)=\gamma_{1} \mathbf{E}_{1}+\gamma_{2} \mathbf{E}_{2}+\gamma_{3} \mathbf{E}_{3}
\end{aligned}
$$

where $m_{i}=M_{i}+c_{i} \gamma_{i}$ for some constants $c_{i}$. It follows from Proposition 2.8 that $L$ is a generic element of $\mathcal{O}_{2}$. A unique non-trivial higher symmetry for the corresponding ODE system corresponds to $A=\pi_{+}(L)$.

\section{Clebsch integrable case}

The factoring subalgebra $\mathcal{U}$ from Example 2.20 generated by elements (2.50) is isotropic and therefore $\mathbf{R}_{i}=\mathbf{E}_{i}, i=1,2,3$. The Lax equation (1.4), (2.56), where and $m_{i}=M_{i}$, is equivalent to (2.53), where

$$
H=-\frac{1}{2}\left(M_{1}^{2}+M_{2}^{2}+M_{3}^{2}-(q+r) \gamma_{1}^{2}-(p+r) \gamma_{2}^{2}-(p+q) \gamma_{3}^{2}\right) .
$$

This coincides with the Clebsch integrable case in the Kirchhoff problem of the motion of a rigid body in an ideal fluid.

Since the subalgebra $\mathcal{U}$ is isotropic, the Lax pair (2.55) gives nothing.

\section{Euler and Steklov-Lyapunov cases}

For the factoring subalgebra from Example 2.21 generated by (2.52) we have

$$
\begin{gathered}
\mathbf{R}_{1}=\mathbf{e}_{1} \frac{1}{\sqrt{(1-r \lambda)(1-q \lambda)} \lambda}, \quad \mathbf{R}_{2}=\mathbf{e}_{2} \frac{1}{\sqrt{(1-r \lambda)(1-p \lambda)} \lambda} \\
\mathbf{R}_{3}=\mathbf{e}_{3} \frac{1}{\sqrt{(1-q \lambda)(1-p \lambda)} \lambda}
\end{gathered}
$$


The Lax pair (2.55) yields the Euler equation (see also Example 1.1)

$$
\mathbf{M}_{t}=\mathbf{M} \times \mathbf{V M}
$$

where $\mathbf{V}=\operatorname{diag}(p, q, r)$. This Lax pair differs from the one considered in Example 1.1.

The Lax equation (1.4), (2.56) is equivalent to (2.53), where

$$
\begin{aligned}
H= & -\frac{1}{2}\left(M_{1}^{2}+M_{2}^{2}+M_{3}^{2}+(r+q) M_{1} \gamma_{1}+(r+p) M_{2} \gamma_{2}+(q+p) M_{3} \gamma_{3}\right) \\
& -\frac{1}{8}\left((r-q)^{2} \gamma_{1}^{2}+(p-r)^{2} \gamma_{2}^{2}+(q-p)^{2} \gamma_{3}^{2}\right)
\end{aligned}
$$

and

$$
m_{1}=M_{1}+\frac{r-q}{2} \gamma_{1}, \quad m_{2}=M_{2}+\frac{p-r}{2} \gamma_{2}, \quad m_{3}=M_{3}+\frac{q-p}{2} \gamma_{3} .
$$

This is just the integrable Steklov-Lyapunov case.

\section{Kirchhoff integrable case}

For the factoring subalgebra described in Example 2.22 the elements

$$
\mathbf{R}_{i}=\frac{\mathbf{e}_{i}}{\lambda}+\nu\left[\mathbf{V}, \mathbf{e}_{i}\right]-\frac{1}{2}\left[\mathbf{V},\left[\mathbf{V}, \mathbf{e}_{i}\right]\right]+(\mathbf{V}, \mathbf{V}) \mathbf{e}_{i}, \quad i=1,2,3
$$

satisfy the commutator relations from Proposition 2.8 and therefore generate a $\mathcal{U}$-module that does not contain non-zero Taylor series. Any such module can be used to construct Lax pairs.

Remark 2.6. This module is not $\mathcal{U}^{\perp}$ (see Remark 2.5).

Exercise 2.8. Find the system of ODEs that corresponds to Lax pair (2.55). Exercise 2.9. Check that the Lax pair (2.56) gives rise to the Kirchhoff integrable case (see [28]).

Open problem 2.3. Find the elements $\mathbf{R}_{i}$ for $\mathcal{U}^{\perp}$.

\subsubsection{Generalization of Euler and Steklov-Lyapunov



The factorizing subalgebra from Example 2.21 can be described by the formula

$$
\mathcal{U}=(\mathbf{1}+\lambda \mathbf{V})^{1 / 2} \mathcal{U}^{s t}(\mathbf{1}+\lambda \mathbf{V})^{1 / 2},
$$

where $\mathbf{V}=\operatorname{diag}(p, q, r)$,

$$
(\mathbf{1}+\lambda \mathbf{V})^{1 / 2}=\mathbf{1}+\frac{1}{2} \mathbf{V} \lambda-\frac{1}{8} \mathbf{V}^{2} \lambda^{2}+\cdots
$$


and $\mathcal{U}^{\text {st }}$ is defined by (2.41). According to Lemma 2.5 the formula (2.57), where $\mathbf{V}$ is arbitrary diagonal matrix, defines a factorizing subalgebra for $\mathcal{G}=\mathfrak{s o}_{n}$ as well. The orthogonal complement to $\mathcal{U}$ is given by

$$
\mathcal{U}^{\perp}=(1+\lambda \mathbf{V})^{-1 / 2} \mathcal{U}^{s t}(1+\lambda \mathbf{V})^{-1 / 2} .
$$

The simplest possibility $L \in \mathcal{O}_{1}, \quad A=\pi_{+}(L)$ corresponds to

$$
\begin{aligned}
& L=(1+\lambda \mathbf{V})^{-1 / 2} \frac{\mathbf{M}}{\lambda}(1+\lambda \mathbf{V})^{-1 / 2}, \\
& A=(1+\lambda \mathbf{V})^{1 / 2} \frac{\mathbf{M}}{\lambda}(1+\lambda \mathbf{V})^{1 / 2},
\end{aligned}
$$

where $\mathbf{M} \in \mathfrak{s o}_{n}$. This Lax pair produces the Euler equation on $\mathfrak{s o}_{n}$ :

$$
\mathbf{M}_{t}=\left[\mathbf{V}, \mathbf{M}^{2}\right] .
$$

The system of equations

$$
\mathbf{M}_{t}=\left[\mathbf{V}, \mathbf{M}^{2}\right]+[\mathbf{M}, \boldsymbol{\Gamma}], \quad \boldsymbol{\Gamma}_{t}=\mathbf{V M} \boldsymbol{\Gamma}-\mathbf{\Gamma M V}, \quad \mathbf{M}, \boldsymbol{\Gamma} \in \mathfrak{s o}_{n}
$$

possesses the Lax pair

$$
\begin{aligned}
& L=(1+\lambda \mathbf{V})^{-1 / 2}\left(\frac{\mathbf{M}}{\lambda^{2}}+\frac{\boldsymbol{\Gamma}}{\lambda}\right)(1+\lambda \mathbf{V})^{-1 / 2}, \\
& A=(1+\lambda \mathbf{V})^{1 / 2} \frac{\mathbf{M}}{\lambda}(1+\lambda \mathbf{V})^{1 / 2},
\end{aligned}
$$

corresponding to the orbit $\mathcal{O}_{2}$. One can regard this equation as an $\mathfrak{s o}_{n^{-}}$ generalization of the Steklov-Lyapunov top [28].

\subsubsection{Factoring subalgebras for Kac-Moody algebras}

The Clebsch, Steklov-Lyapunov and Kirchhoff ${ }^{2}$ cases possess additional integrals of second degree. To get trickier examples like Kowalevsky top, one can consider a decomposition problem for Kac-Moody algebras (see [28]).

Let $\mathcal{G}$ be a semi-simple Lie algebra and $\phi$ be an automorphism of $\mathcal{G}$ of a finite order $k$. Let

$$
\mathcal{G}_{i}=\left\{a \in \mathcal{G} \mid \quad \phi(a)=\varepsilon^{i} a\right\} \lambda^{i}, \quad i \in \mathbb{Z},
$$

where $\varepsilon$ is a primitive root of 1 of degree $k$. In this case, the Lie algebra

$$
\mathcal{G}((\lambda, \phi))=\left\{\sum_{i=-n}^{\infty} g_{i} \mid \quad g_{i} \in \mathcal{G}_{i}, \quad n \in \mathbb{Z}\right\}
$$

\footnotetext{
${ }^{2}$ For the Kirchhoff case there exists also an integral of first degree.
} 
is $\mathbb{Z}$-graded. It is called an (extended) twisted loop algebra or a Kac-Moody algebra.

Several interesting integrable systems are related to the following KacMoody algebra. Let

$$
\mathcal{G}=\left\{\mathbf{A} \in \operatorname{Mat}_{n+m} \mid \mathbf{A}^{t}=-\mathbf{S A} \mathbf{S}\right\}
$$

where

$$
\mathbf{S}=\left(\begin{array}{cc}
\mathbf{1}_{n} & 0 \\
0 & -\mathbf{1}_{m}
\end{array}\right)
$$

It is clear that the Lie algebra $\mathcal{G}$ is isomorphic over $\mathbb{C}$ to $\mathfrak{s o}_{n+m}$.

Consider the subalgebra $\mathcal{A}$ of the loop algebra over $\mathcal{G}$ consisting of Laurent series such that the coefficients of even (respectively, odd) powers of $\lambda$ belong to $\mathcal{G}_{1}$ (respectively, $\mathcal{G}_{-1}$ ). Here by $\mathcal{G}_{ \pm 1}$ we denote the eigenspaces of the inner second order automorphism $\phi: \mathcal{G} \rightarrow \mathbf{S} \mathcal{G} \mathbf{S}^{-1}$, corresponding to eigenvalues \pm 1 . Actually, this means that the coefficients of even powers of $\lambda$ have the following block structure

$$
\left(\begin{array}{cc}
v_{1} & 0 \\
0 & v_{2}
\end{array}\right)
$$

where $v_{1} \in \mathfrak{s o}_{n}, v_{2} \in \mathfrak{s o}_{m}$, and the coefficients of odd powers are of the form

$$
\left(\begin{array}{cc}
0 & w \\
w^{t} & 0
\end{array}\right)
$$

where $w \in$ Mat $_{n, m}$.

We choose res $\left(\lambda^{-1} \operatorname{tr}(X Y)\right)$ for the non-degenerate invariant form on $\mathcal{A}$. Note that in this case the form $\operatorname{res}(\operatorname{tr}(X Y))$ is degenerate.

Let $\mathcal{T}$ be the set of all Taylor series from $\mathcal{A}$,

$$
\mathcal{U}=(\mathbf{1}+\lambda r)^{1 / 2} \mathcal{U}^{s t}(\mathbf{1}+\lambda r)^{1 / 2},
$$

where $\mathcal{U}^{s t}$ is the set of polynomials in $\lambda^{-1}$ from $\mathcal{A}$ and $r$ is arbitrary constant matrix of the form

$$
r=\left(\begin{array}{cc}
0 & r_{1} \\
-r_{1}^{t} & 0
\end{array}\right), \quad r_{1} \in \operatorname{Mat}_{n, m} .
$$

According to Lemma $2.5, \mathcal{U}$ is a factoring subalgebra and the sum $\mathcal{A}=$ $\mathcal{T}+\mathcal{U}$ is direct. The subalgebra $\mathcal{U}$ is a natural generalization of (2.57) to the case when the structure of coefficients of series from $\mathcal{U}$ are defined by an additional automorphism of second order. The orthogonal complement to $\mathcal{U}$ with respect of the form

$$
\langle X, Y\rangle=\operatorname{res} \lambda^{-1} \operatorname{tr}(X Y)
$$


is given by

$$
\mathcal{U}^{\perp}=(\mathbf{1}+\lambda r)^{-1 / 2} \mathcal{U}^{s t}(\mathbf{1}+\lambda r)^{-1 / 2} .
$$

The Lax equation $L_{t}=\left[\pi_{+}(L), L\right]$ corresponding to

$$
L=(\mathbf{1}+\lambda r)^{-1 / 2}\left(\lambda^{-1} w+v+\lambda u\right)(\mathbf{1}+\lambda r)^{-1 / 2}
$$

is equivalent to the following system of equations

$$
\begin{aligned}
& w_{t}=[w, w r+r w-v], \\
& v_{t}=[u, w]+v w r-r w v, \\
& u_{t}=u w r-r w u .
\end{aligned}
$$

It is easy to see that this system admits the reduction

$$
u=\left(\begin{array}{cc}
0 & r_{1} \\
r_{1}^{t} & 0
\end{array}\right),
$$

which leads to the model found in [79]. In the case $n=3, m=2$ under further reductions we arrive at the Lax representation for the integrable case in the Kirchhoff problem [71] with the Hamiltonian

$$
H=\frac{1}{2}|\mathbf{u}|^{2}|\mathbf{M}|^{2}+\frac{1}{2}(\mathbf{u}, \mathbf{M})^{2}+(\mathbf{u} \times \mathbf{v}, \mathbf{M} \times \mathbf{\Gamma}),
$$

where $\mathbf{u}$ and $\mathbf{v}$ are arbitrary constant vectors such that $(\mathbf{u}, \mathbf{v})=0$. The additional integral of motion in this case is of degree four.

\subsubsection{Integrable PDEs of the Landau-Lifshitz type}

\section{Landau-Lifshitz equations related to $\mathfrak{s o}_{3}$}

Any factoring subalgebra $\mathcal{U}$ for $\mathfrak{s o}_{3}$ yields the following Lax pair

$$
\begin{gathered}
L=\frac{d}{d x}+U, \quad U=\sum_{i=1}^{3} s_{i} \mathbf{E}_{i}, \quad s_{1}^{2}+s_{2}^{2}+s_{3}^{2}=1, \\
A=\sum s_{i}\left[\mathbf{E}_{j}, \mathbf{E}_{k}\right]+\sum t_{i} \mathbf{E}_{i}
\end{gathered}
$$

for an integrable PDE of the Landau-Lifshitz type. In this case the Lax equation has the form

$$
U_{t}-A_{x}+[U, A]=0 .
$$

The Laurent expansion of the left hand side of (2.59) contains terms with $\lambda^{k}$, where $k \geq-2$. If coefficients at $\lambda^{-2}$ and at $\lambda^{-1}$ vanish, then the left 
hand side of (2.59) identically equals zero. Indeed, the subalgebra $\mathcal{U}$ does not contain any non-zero Taylor series.

To find the corresponding non-linear system of the form $\mathbf{s}_{t}=$ $\vec{F}\left(\mathbf{s}, \mathbf{s}_{x}, \mathbf{s}_{x x}\right)$, where $\mathbf{s}=\left(s_{1}, s_{2}, s_{3}\right)$, one can use the following straightforward computation. Comparing the coefficients of $\lambda^{-2}$, we express $t_{i}$ in terms of $\mathbf{s}, \mathbf{s}_{x}$. Equating the coefficients of $\lambda^{-1}$, we get an evolution system for $\mathbf{s}$. Using the symmetry approach to integrability [50] these systems were found in [49].

A standard way of finding all $A$-operators of the hierarchy defined by a given $L$-operator of the Landau-Lifshitz type based on a diagonalization procedure (cf. with Theorem 2.6) was proposed in [29]. Here we don't discuss it.

Consider the case of Example 2.20. Equating the coefficients of $\lambda^{-2}$ in (2.59) to zero, we get $\mathbf{s}_{x}=\mathbf{s} \times \mathbf{t}$, where $\mathbf{t}=\left(t_{1}, t_{2}, t_{3}\right)$. Since $\mathbf{s}^{2}=1$ we find $\mathbf{t}=\mathbf{s}_{x} \times \mathbf{s}+\mu \mathbf{s}$. Comparing the coefficients of $\lambda^{-1}$, we get $\mathbf{s}_{t}=\mathbf{t}_{x}-\mathbf{s} \times \mathbf{V s}$ or

$$
\mathbf{s}_{t}=\mathbf{s}_{x x} \times \mathbf{s}+\mu_{x} \mathbf{s}+\mu \mathbf{s}_{x}-\mathbf{s} \times \mathbf{V} \mathbf{s},
$$

where $\mathbf{V}=\operatorname{diag}(p, q, r)$. Since the scalar product $\left(\mathbf{s}, \mathbf{s}_{t}\right)$ has to be zero, we find that $\mu=$ const. The resulting equation coincides with (2.37) up to the involution $t \rightarrow-t$, the additional term of the form const $\mathbf{s}_{x}$ and a change of notation.

The factoring subalgebra from Example 2.21 yields the equation

$$
\mathbf{s}_{t}=\mathbf{s} \times \mathbf{s}_{x x}+(\mathbf{s}, \mathbf{V} \mathbf{s}) \mathbf{s}_{x}+2 \mathbf{s} \times\left(\mathbf{s} \times \mathbf{V} \mathbf{s}_{x}\right) .
$$

The subalgebra from Example 2.22 corresponds to equation

$$
\mathbf{s}_{t}=\mathbf{s} \times \mathbf{s}_{x x}+(\mathbf{s}, \mathbf{Z} \mathbf{s}) \mathbf{s}_{x}+2 \mathbf{s} \times\left(\mathbf{s} \times \mathbf{Z} \mathbf{s}_{x}\right)+c \mathbf{s} \times \mathbf{Z} \mathbf{s},
$$

where

$$
\mathbf{Z}=\left(\begin{array}{ccc}
r_{1}^{2} & r_{1} r_{2} & r_{1} r_{3} \\
r_{1} r_{2} & r_{2}^{2} & r_{2} r_{3} \\
r_{1} r_{3} & r_{2} r_{3} & r_{3}^{2}
\end{array}\right), \quad c=\nu^{2}+\frac{r_{1}^{2}+r_{2}^{2}+r_{3}^{2}}{4}
$$

In this equation $\mathbf{Z}$ is an arbitrary symmetric matrix of rank one and $c$ is an arbitrary constant.

\section{Perelomov model and vector Landau-Lifshitz equation}

Consider a special case $n=N, m=1$ of the Kac-Moody algebra from Section 2.3.5. Let us take

$$
\mathcal{U}=\left\{\sum_{i=-n}^{0} \lambda^{2 i}\left(\begin{array}{cc}
\Lambda \mathbf{A}_{i} \Lambda & \Lambda \mathbf{u}_{i} \\
\mathbf{u}_{i}^{t} \Lambda & 0
\end{array}\right), \quad n \in \mathbb{N}\right\}
$$


for the factoring subalgebra. Here

$$
\Lambda=\frac{1}{\lambda} \sqrt{\mathbf{1 - \lambda ^ { 2 } \mathbf { R }}}=\frac{\mathbf{1}}{\lambda}-\frac{\mathbf{R}}{2} \lambda-\frac{\mathbf{R}^{2}}{8} \lambda^{3}+\cdots,
$$

$\mathbf{R}=\operatorname{diag}\left(r_{1}, \ldots, r_{N}\right), \mathbf{A}_{i}$ are skew-symmetric $N \times N$-matrices, and $\mathbf{u}_{i}$ are column vectors. The orthogonal complement to $\mathcal{U}$ with respect to the form $\operatorname{res}\left(\lambda^{-1}(X, Y)\right)$ has the form

$$
\mathcal{U}^{\perp}=\left\{\sum_{i=-n}^{-1} \lambda^{2 i}\left(\begin{array}{cc}
\Lambda^{-1} \mathbf{A}_{i} \Lambda^{-1} & \Lambda^{-1} \mathbf{u}_{i} \\
\mathbf{u}_{i}^{t} \Lambda^{-1} & 0
\end{array}\right), \quad n \in \mathbb{N}\right\} .
$$

The simplest $L$-operator

$$
L=\frac{1}{\lambda^{2}}\left(\begin{array}{cc}
\Lambda^{-1} \mathbf{V} \Lambda^{-1} & \Lambda^{-1} \mathbf{u} \\
\mathbf{u}^{t} \Lambda^{-1} & 0
\end{array}\right)
$$

corresponds to $n=1$ in (2.60). The Lax equation (1.4) with

$$
A=\pi_{+}\left(\lambda^{-2} L\right)=\frac{1}{\lambda^{2}}\left(\begin{array}{cc}
0 & \Lambda \mathbf{u} \\
\mathbf{u}^{t} \Lambda & 0
\end{array}\right)+\left(\begin{array}{cc}
\Lambda \mathbf{V} \Lambda & \Lambda \mathbf{R u} \\
\mathbf{u}^{t} \mathbf{R} \Lambda & 0
\end{array}\right)
$$

gives rise to Perelomov's $\mathfrak{s o}_{N}$ generalization

$$
\mathbf{V}_{t}=\left[\mathbf{V}^{2}, \mathbf{R}\right]+\left[\mathbf{u u}^{t}, \mathbf{R}^{2}\right], \quad \mathbf{u}_{t}+(\mathbf{V R}+\mathbf{R V}) \mathbf{u}=0
$$

of the Clebsch top system.

For the Landau-Lifshitz equation (2.36) the $L$-operator is given by (2.38) and

$$
A=\frac{1}{\lambda^{2}}\left(\begin{array}{cc}
0 & \Lambda \mathbf{u} \\
\mathbf{u}^{T} \Lambda & 0
\end{array}\right)+\left(\begin{array}{cc}
\Lambda \mathbf{V} \Lambda & \Lambda \mathbf{y} \\
\mathbf{y}^{t} \Lambda & 0
\end{array}\right)
$$

where the entries of $\mathbf{V}$ are given by $v_{i, j}=u_{i}\left(u_{j}\right)_{x}-u_{j}\left(u_{i}\right)_{x}$ and

$$
\mathbf{y}=\mathbf{u}_{x x}+\left(\frac{3}{2}\left\langle\mathbf{u}_{x}, \mathbf{u}_{x}\right\rangle+\frac{1}{2}\langle\mathbf{u}, \mathbf{R u}\rangle\right) \mathbf{u} .
$$

\subsubsection{Hyperbolic models of chiral type}

A class of factoring subalgebras for $\mathcal{G}=\mathfrak{s o}_{4}$ and their relations with integrable $\mathfrak{s o}_{4}$ spinning tops were investigated in [15]. These subalgebras also generate $[26,16]$ integrable hyperbolic PDEs of the form

$$
\mathbf{u}_{\xi}=\mathbf{A v} \times \mathbf{u}, \quad \mathbf{v}_{\eta}=\overline{\mathbf{A}} \mathbf{u} \times \mathbf{v},
$$

where

$$
\mathbf{A}=\operatorname{diag}\left(a_{1}, a_{2}, a_{3}\right), \quad \overline{\mathbf{A}}=\operatorname{diag}\left(\bar{a}_{1}, \bar{a}_{2}, \bar{a}_{3}\right),
$$


$\mathbf{u}, \mathbf{v}$ are three-dimensional $\mathrm{s}$, and the constants $a_{i}, \bar{a}_{j}$ obey the following relations.

$$
\begin{aligned}
& a_{1} \bar{a}_{1}\left(a_{3}^{2}-a_{2}^{2}\right)+a_{2} \bar{a}_{2}\left(a_{1}^{2}-a_{3}^{2}\right)+a_{3} \bar{a}_{3}\left(a_{2}^{2}-a_{1}^{2}\right)=0, \\
& a_{1} \bar{a}_{1}\left(\bar{a}_{3}^{2}-\bar{a}_{2}^{2}\right)+a_{2} \bar{a}_{2}\left(\bar{a}_{1}^{2}-\bar{a}_{3}^{2}\right)+a_{3} \bar{a}_{3}\left(\bar{a}_{2}^{2}-\bar{a}_{1}^{2}\right)=0 .
\end{aligned}
$$

The Cherednik model [8] corresponds to $a_{i}=\bar{a}_{i}, \quad i=1,2,3$. For the Golubchik-Sokolov case [26] we have $a_{i}=\bar{a}_{i}^{-1}, \quad i=1,2,3$.

The case when $a_{1}=a_{2}$ and then $\bar{a}_{1}=\bar{a}_{2}, a_{3}, \bar{a}_{3}$ are arbitrary, was found in $[16]$.

\section{Resume}

The description of factoring subalgebras is a fundamental problem of the theory. Each factoring subalgebra generates several different integrable PDEs and ODEs.

\subsection{Factorization method and non-associative algebras}

Let $\mathcal{A}$ be an $N$-dimensional algebra with a multiplication operation $\circ$ defined by the structural constants $C_{j k}^{i}$. We associate to $\mathcal{A}$ a top-like ODEsystem of the form

$$
u_{t}^{i}=\sum_{j, k} C_{j k}^{i} u^{j} u^{k}, \quad i, j, k=1, \ldots, N .
$$

Let $\mathbf{e}_{1}, \ldots, \mathbf{e}_{\mathbf{N}}$ be a basis in $\mathcal{A}$ and

$$
U=\sum_{i=1}^{N} u_{i} \mathbf{e}_{i}
$$

The system (2.61) can be written in a short form

$$
U_{t}=U \circ U
$$

The system (2.62) is called the $\mathcal{A}$-top.

Definition 2.5. Algebras with the identity $[X, Y, Z]=0$ are called leftsymmetric [84].

Hereinafter we use the notation (1.2) and (1.3).

Definition 2.6. Algebras with the identity

$$
[V, X, Y \circ Z]-[V, X, Y] \circ Z-Y \circ[V, X, Z]=0 .
$$

are called SS-algebras $[78,34]$. 
Remark 2.7. It follows from (2.63) that for any $S S$-algebra $\mathcal{A}$ the operator

$$
K_{Y Z}=\left[L_{Y}, L_{Z}\right]-L_{Y \circ Z}+L_{Z \circ Y}
$$

is a derivation of $\mathcal{A}$ for any $Y, Z$. As usual, $L_{X}$ denotes the operator of left multiplication by $X$.

Definition 2.7. An algebra with identities

$$
[X, Y, Z]+[Y, Z, X]+[Z, X, Y]=0,
$$

and

$$
V \circ[X, Y, Z]=[V \circ X, Y, Z]+[X, V \circ Y, Z]+[X, Y, V \circ Z]
$$

is called G-algebra [30].

Remark 2.8. Identity (2.64) means that the operation $X \circ Y-Y \circ X$ is a Lie bracket.

\subsubsection{Factorization method}

The factorization method (or, the same, AKS-scheme [39]) is a finitedimensional analog of the Riemann-Hilbert problem [89], which can be used as a basis for the inverse scattering method.

Similarly to Section 2.3, we deal with a vector space decomposition of a Lie algebra into a direct sum of its subalgebras.

Let $\mathcal{G}$ be a finite-dimensional Lie algebra, $\mathcal{G}_{+}$and $\mathcal{G}_{-}$be subalgebras in $\mathcal{G}$ such that

$$
\mathcal{G}=\mathcal{G}_{+} \oplus \mathcal{G}_{-} .
$$

The simplest example is the Gauss decomposition of the matrix algebra into the sum of upper and law triangular matrices.

The standard factorization method is used to integrate the following very special systems of the form (2.61):

$$
X_{t}=\left[\pi_{+}(X), X\right], \quad X(0)=x_{0} .
$$

Here $X(t) \in \mathcal{G}, \pi_{+}$is the projector onto $\mathcal{G}_{+}$parallel to $\mathcal{G}_{-}$. Very often we denote by $X_{+}$and $X_{-}$the projections of $X$ onto $\mathcal{G}_{+}$and $\mathcal{G}_{-}$, respectively. For simplicity we assume that $\mathcal{G}$ is embedded into a matrix algebra.

Remark 2.9. It follows from Lemma 1.1 that for any $k$ the function $\operatorname{tr} X^{k}$ is an integral of motion for (2.67).

Proposition 2.9. The solution of Cauchy problem (2.67) is given by the formula

$$
X(t)=A(t) x_{0} A^{-1}(t),
$$


where function $A(t)$ is defined as a solution of the following factorization problem

$$
A^{-1} B=\exp \left(-x_{0} t\right), \quad A \in G_{+}, \quad B \in G_{-},
$$

where $G_{+}$and $G_{-}$are the Lie groups of $\mathcal{G}_{+}$and $\mathcal{G}_{-}$, respectively.

Proof. Differentiating (2.68), we obtain

$$
X_{t}=A_{t} x_{0} A^{-1}-A x_{0} A^{-1} A_{t} A^{-1}=\left[A_{t} A^{-1}, X\right] .
$$

It follows from (2.69) that

$$
-A^{-1} A_{t} A^{-1} B+A^{-1} B_{t}=-x_{0} A^{-1} B .
$$

The latter relation is equivalent to

$$
-A_{t} A^{-1}+B_{t} B^{-1}=-A x_{0} A^{-1} .
$$

Projecting it onto $\mathcal{G}_{+}$, we get $A_{t} A^{-1}=X_{+}$which proves $(2.67)$.

If the groups $G_{+}$and $G_{-}$are algebraic, then the conditions

$$
A \in G_{+}, \quad A \exp \left(-x_{0} t\right) \in G_{-}
$$

are equivalent to a system of algebraic equations from which (for small $t$ ) the matrix $A(t)$ is uniquely determined.

The factorization problem (2.69) can also be reduced to a system of linear differential equations with variable coefficients for $A(t)$. Define a linear operator $L(t): \mathcal{G}_{+} \rightarrow \mathcal{G}_{+}$by the formula

$$
L(t)(v)=\left(\exp \left(x_{0} t\right) v \exp \left(-x_{0} t\right)\right)_{+} .
$$

Since $L(0)$ is the identity operator, $L(t)$ is invertible for small $t$.

Proposition 2.10. Let $A(t)$ be the solution of the initial problem

$$
A_{t}=A L(t)^{-1}\left(\left(x_{0}\right)_{+}\right), \quad A(0)=I .
$$

Define $B$ by the formula $B=A \exp \left(-x_{0} t\right)$. Then the pair $(A, B)$ is the solution of the factorization problem (2.69).

Proof. Since $A^{-1} A_{t} \in \mathcal{G}_{+}$and $A(0)=I$, we have $A \in G_{+}$. It suffices to verify that $B^{-1} B_{t} \in \mathcal{G}_{-}$. We have

$$
\begin{aligned}
B^{-1} B_{t}= & \exp \left(x_{0} t\right) A^{-1}\left(A_{t} \exp \left(-x_{0} t\right)-A x_{0} \exp \left(-x_{0} t\right)\right)= \\
& \exp \left(x_{0} t\right)\left(L(t)^{-1}\left(x_{0}\right)_{+}\right) \exp \left(-x_{0} t\right)-x_{0} .
\end{aligned}
$$

Projecting this identity onto $\mathcal{G}_{+}$and using the definition of the operator $L(t)$, we obtain $\left(B^{-1} B_{t}\right)_{+}=0$. 


\subsubsection{Reductions}

It follows from (2.68) that if the initial data $x_{0}$ for (2.67) belongs to a $\mathcal{G}_{+}$-module $\mathcal{M}$, then $X(t) \in \mathcal{M}$ for any $t$. Such a specialization of $(2.67)$ can be written as

$$
M_{t}=\left[\pi_{+}(M), M\right], \quad M \in \mathcal{M}
$$

Introducing the product

$$
M_{1} \circ M_{2}=\left[\pi_{+}\left(M_{1}\right), M_{2}\right], \quad M_{i} \in \mathcal{M},
$$

we equip $\mathcal{M}$ with a structure of algebra. The system $(2.70)$ is called $\mathcal{M}$ reduction and the operation $(2.71)$ is called $\mathcal{M}$-product.

Some classes of modules $\mathcal{M}$ correspond to interesting non-associative algebras defined by (2.71).

\section{Reductions for $\mathbb{Z}_{\mathbf{2}}$-graded Lie algebras}

Let

$$
\mathcal{G}=\mathcal{G}_{0} \oplus \mathcal{G}_{1}
$$

be a $\mathbb{Z}_{2}$-graded Lie algebra:

$$
\left[\mathcal{G}_{0}, \mathcal{G}_{0}\right] \subset \mathcal{G}_{0}, \quad\left[\mathcal{G}_{0}, \mathcal{G}_{1}\right] \subset \mathcal{G}_{1}, \quad\left[\mathcal{G}_{1}, \mathcal{G}_{1}\right] \subset \mathcal{G}_{0}
$$

Suppose that we have a decomposition (2.66), where $\mathcal{G}_{+}=\mathcal{G}_{0}$. Let us consider the $\mathcal{G}_{1}$-reduction.

Example 2.23. Let $\mathcal{G}_{0}=\mathcal{G}_{+}, \mathcal{G}_{1}=\mathcal{M}, \mathcal{G}_{-}$, and $\mathcal{G}$ be the sets of skewsymmetric, symmetric, upper-triangular and all matrices, respectively. Then the formula (2.71) defines the structure of a $G$-algebra on the set of symmetric matrices.

It is clear that

$$
\mathcal{G}_{-}=\left\{m-R(m) \mid m \in \mathcal{G}_{1}\right\},
$$

where $R=\pi_{+}$is the projection onto $\mathcal{G}_{+}=\mathcal{G}_{0}$ parallel to $\mathcal{G}_{-}$.

Theorem 2.9. [30] The vector space in (2.73) is a Lie subalgebra in $\mathcal{G}$ iff $R: \mathcal{G}_{1} \rightarrow \mathcal{G}_{0}$ satisfies the modified Yang-Baxter equation

$R([R(X), Y]-[R(Y), X])-[R(X), R(Y)]-[X, Y]=0, \quad X, Y \in \mathcal{G}_{1}$.

Remark 2.10. It is important to note that in our case $R$ is an operator defined on $\mathcal{G}_{1}$ and acting from $\mathcal{G}_{1}$ to $\mathcal{G}_{0}$, whereas usually (see [68]) $R$ is assumed to be an operator on $\mathcal{G}$.

Proposition 2.11. If $\left[\mathcal{G}_{1}, \mathcal{G}_{1}\right]=\{0\}$, then $\mathcal{G}_{1}$ is a left-symmetric algebra with respect to the product (2.71). 
Proof. Let $X, Y, Z \in \mathcal{G}_{1}$. Let us verify that

$$
[X, Y, Z]=[[X, Y], Z]
$$

where the left hand side is defined by (1.3). According to (2.71), it has the form

$$
\left[\left[X_{+}, Y\right]_{+}, Z\right]-\left[\left[Y_{+}, X\right]_{+}, Z\right]+\left[Y_{+},\left[X_{+}, Z\right]\right]-\left[X_{+},\left[Y_{+}, Z\right]\right] .
$$

We have

$$
\begin{gathered}
{\left[\left[X_{+}, Y\right]_{+}, Z\right]-\left[\left[Y_{+}, X\right]_{+}, Z\right]=\left[[X, Y]_{+}, Z\right]-\left[\left[X_{-}, Y\right]_{+}, Z\right]+\left[\left[X, Y_{+}\right]_{+}, Z\right]=} \\
{[[X, Y], Z]+\left[\left[X_{+}, Y_{+}\right]_{+}, Z\right]=[[X, Y], Z]+\left[\left[X_{+}, Y_{+}\right], Z\right] .}
\end{gathered}
$$

Now (2.74) follows from the Jacobi identity for $X_{+}, Y_{+}, Z$. Since $\left[\mathcal{G}_{1}, \mathcal{G}_{1}\right]=$ $\{0\}$ the proposition statement is a consequence of (2.74).

In the general case we arrive at $G$-algebras.

Theorem 2.10. $\quad$ i) The vector space $\mathcal{G}_{1}$ is a $G$-algebra with respect to the operation (2.71).

ii) Any $G$-algebra can be obtained from a suitable $\mathbb{Z}_{2}$-graded Lie algebra by the above construction.

Proof. To prove identity (2.64) it suffices to project the Jacobi identity for $X_{-}, Y_{-}, Z_{-}$onto $\mathcal{G}_{1}$. Rewriting (2.65) in terms of the $\mathcal{G}$-bracket with the help of (2.74), we see that (2.65) follows from the Jacobi identity for $\mathcal{G}$.

It remains to prove the second part of the theorem. Let $\mathcal{G}_{1}$ be a $G$-algebra. Define $\mathcal{G}$ by formula $(2.72)$, where $G_{0}$ is the Lie algebra generated by all operators of left multiplication of $\mathcal{G}_{1}$. Recall that the left multiplication operator $L_{X}$ is defined as follows: $L_{X}(Y)=X \circ Y$. The bracket on $\mathcal{G}$ is defined by

$$
[(A, X),(B, Y)]=\left([A, B]-\left[L_{X}, L_{Y}\right]+L_{X \circ Y}-L_{Y \circ X}, A(Y)-B(X)\right) .
$$

The skew-symmetry is obvious. One can easily verify that the identities (2.64), (2.65) are equivalent to the Jacobi identity for (2.75). It follows from (2.75) that the decomposition (2.72) defines a $\mathbb{Z}_{2}$-gradation. To define a decomposition $(2.66)$ we take for $\mathcal{G}_{-}$the set $\left\{\left(-L_{X}, X\right)\right\}$ and $\mathcal{G}_{0}$ for $\mathcal{G}_{+}$. Formula (2.75) implies that $\mathcal{G}_{-}$is a subalgebra in $\mathcal{G}$. For $\mathcal{G}_{ \pm}$thus defined, (2.71) has the form $(0, X) \circ(0, Y)=\left[\left(L_{X}, 0\right),(0, Y)\right]$. This relation is fulfilled according to $(2.75)$.

The part i) of the theorem means that any $G$-top (2.61) is integrable by the factorization method. 
Example 2.24. Putting

$$
\mathcal{G}_{+}=\left\{\left(\begin{array}{ccc}
a & c & 0 \\
d & b & 0 \\
0 & 0 & -a-b
\end{array}\right)\right\}, \quad \mathcal{G}_{1}=\left\{\left(\begin{array}{ccc}
0 & 0 & P \\
0 & 0 & Q \\
R & S & 0
\end{array}\right)\right\},
$$

we take $\mathfrak{s l}_{3}$ for $\mathcal{G}$. Let us choose a complementary subalgebra $\mathcal{G}_{-}$as follows:

$$
\mathcal{G}_{-}=\left\{\left(\begin{array}{ccc}
-Y+X+\alpha U & X & Y-X \\
-Z+(2-3 \alpha) U & (1-2 \alpha) U & Z+(3 \alpha-2) U \\
-Y & X & Y-X+(\alpha-1) U
\end{array}\right)\right\},
$$

where $\alpha$ is a parameter.

The operation (2.71) turns the vector space $\mathcal{M}=\mathcal{G}_{1}$ into a $G$-algebra. The corresponding $\mathcal{M}$-top is the following system of differential equations

$$
\left\{\begin{array}{l}
P_{t}=P^{2}-R P-Q S, \\
Q_{t}=(\beta-2) R Q+\beta P Q, \\
R_{t}=R^{2}-R P-Q S, \\
S_{t}=(3-\beta) R S+(1-\beta) P S,
\end{array}\right.
$$

where $\beta=3 \alpha$, for the entries of the matrix

$$
\mathbf{M}=\left(\begin{array}{lll}
0 & 0 & P \\
0 & 0 & Q \\
R & S & 0
\end{array}\right)
$$

From (2.70) it follows that $I_{1}=\operatorname{tr} \mathbf{M}^{2}=R P+Q S$ is a first integral for the system. Other integrals of the form $\operatorname{tr} \mathbf{M}^{k}$ are trivial. Nevertheless it is not hard to integrate (2.76) by quadratures. The auxiliary two first integrals are of the form

$$
I_{2}=\frac{P-R}{Q S}, \quad I_{3}=Q^{1-\beta} S^{-\beta}\left(R^{2}-R P-Q S\right) .
$$

For generic $\beta$ the integral $I_{3}$ is a multi-valued function. It shows that (2.76) is not integrable from the view-point of the Painleve approach (see for example, [53]).

\subsubsection{Generalized factorization method}

Suppose that

$$
\mathcal{G}=V_{1} \oplus V_{2},
$$

where the $V_{i}$ are vector spaces. Let

$$
\mathcal{H}=\left[V_{1}, V_{1}\right]_{-}+\left[V_{2}, V_{2}\right]_{+} .
$$


Here + and - symbolize the projections onto $V_{1}$ and $V_{2}$, respectively. Assume that $V_{1}$ and $V_{2}$ satisfy the following conditions

$$
\left[\mathcal{H}, V_{1}\right] \subset V_{1}, \quad\left[\mathcal{H}, V_{2}\right] \subset V_{2} .
$$

If $V_{i}$ are subalgebras, then $\mathcal{H}=\{0\}$ and conditions (2.79) are trivial.

It turns out [33] that equation (2.67), where $\pi_{+}$is the projection onto $V_{1}$ parallel to $V_{2}$, can be reduced to solving a system of linear equations with variable coefficients (cf. Proposition 2.10).

Remark 2.11. If conditions (2.79) hold, then $\mathcal{H}, \mathcal{G}_{+}=V_{1}+\left[V_{1}, V_{1}\right]_{-}$and $\mathcal{G}_{-}=V_{2}+\left[V_{2}, V_{2}\right]_{+}$are Lie subalgebras in $\mathcal{G}$. Moreover, $\mathcal{H}=\mathcal{G}_{+} \bigcap \mathcal{G}_{-}$

Theorem 2.11. $\quad$ i) Let $\mathcal{G}=\mathcal{G}_{0} \oplus \mathcal{G}_{1}$ be a $\mathbb{Z}_{2}$-graded Lie algebra, such that $\left[\mathcal{G}_{1}, \mathcal{G}_{1}\right]=0$. Given a vector space decomposition (2.77) with $V_{1}=\mathcal{G}_{0}$ and a vector space $V_{2}$ satisfying conditions (2.79), we equip $V_{2}$ with an algebraic structure by formula (2.71). Then $V_{2}$ is a SSalgebra with respect to the operation 0 .

ii) Any $S S$-algebra $\mathcal{A}$ can be obtained from a suitable $\mathbb{Z}_{2}$-graded Lie algebra by the above construction

Proof. The first part can be proved in the same manner as the first part of Theorem 2.10. We explain only how to construct $\mathcal{G}, \mathcal{G}_{+}, V_{2}$. for a given $S S$ algebra. We take for $\mathcal{G}_{+}$the Lie algebra End $\mathcal{A}$ of all linear endomorphisms of $\mathcal{A}$. The vector space

$$
\mathcal{G}=(\operatorname{End} \mathcal{A}) \oplus \mathcal{A}
$$

becomes a $\mathbb{Z}_{2}$-graded Lie algebra if we define the bracket by

$$
[(A, X),(B, Y)]=([A, B], A(Y)-B(X)) .
$$

It is not difficult to show that (2.63) implies that a) the vector space $\mathcal{H}$ generated by all elements of the form

$$
\left(\left[L_{Y}, L_{Z}\right]-L_{Y \circ Z}+L_{Z \circ Y}, 0\right)
$$

is a Lie subalgebra in $\mathcal{G}$, and b) the vector spaces $V_{2}=\left\{\left(-L_{X}, X\right)\right\}$, $V_{1}=\mathcal{G}_{+}$and the subalgebra $\mathcal{H}$ satisfy conditions (2.78) and (2.79).

Example 2.25. Let us take

$$
\mathcal{G}=\left\{\left(\begin{array}{llll}
* & * & * & * \\
* & * & * & * \\
* & * & * & * \\
0 & 0 & 0 & 0
\end{array}\right)\right\}
$$


for $\mathbb{Z}_{2}$-graded Lie algebra. It is clear that $\mathcal{G}=\mathcal{G}_{0} \oplus \mathcal{G}_{1}$, where

$$
\mathcal{G}_{0}=\left\{\left(\begin{array}{llll}
* & * & * & 0 \\
* & * & * & 0 \\
* & * & * & 0 \\
0 & 0 & 0 & 0
\end{array}\right)\right\}, \quad \mathcal{G}_{1}=\left\{\left(\begin{array}{cccc}
0 & 0 & 0 & P \\
0 & 0 & 0 & Q \\
0 & 0 & 0 & R \\
0 & 0 & 0 & 0
\end{array}\right)\right\} .
$$

Let $\mathcal{G}_{+}=\mathcal{G}_{0}$ and

$$
\mathcal{G}_{-}=\left\{\left(\begin{array}{cccc}
c & \lambda c & a & a \\
-\lambda c & c & b & b \\
a & b & c & c \\
0 & 0 & 0 & 0
\end{array}\right)\right\}
$$

where $\lambda$ is a parameter. Since $\mathcal{G}_{-}$is not a subalgebra, we have to find the vector space $\mathcal{H}$ using (2.78). A simple calculation shows that

$$
\mathcal{H}=\left\{\left(\begin{array}{cccc}
0 & -d & 0 & 0 \\
d & 0 & 0 & 0 \\
0 & 0 & 0 & 0 \\
0 & 0 & 0 & 0
\end{array}\right)\right\}
$$

and that the conditions (2.79) are fulfilled. The corresponding $S S$-top (up to a scaling) is given by

$$
\left\{\begin{array}{l}
P_{t}=2 P R+\lambda Q R \\
Q_{t}=2 Q R-\lambda P R \\
R_{t}=P^{2}+Q^{2}+R^{2} .
\end{array}\right.
$$




\section{Chapter 3}

\section{Algebraic structures in bi-Hamiltonian approach}

\subsection{Polynomial forms for elliptic Calogero- Moser systems}

\subsubsection{Calogero-Moser Hamiltonians}

Consider quantum integrable Hamiltonians of the form

$$
H=-\Delta+U\left(x_{1}, \ldots, x_{n}\right), \quad \text { where } \quad \Delta=\sum_{i=1}^{n} \frac{\partial^{2}}{\partial x_{i}^{2}}
$$

related to simple Lie algebras [59]. For such Hamiltonians the potential $U$ is a rational, trigonometric or elliptic function.

Observation 3.1. (A.Turbiner). For many of these Hamiltonians there exists a change of variables and a gauge transformation that bring the Hamiltonian to a differential operator with polynomial coefficients.

The elliptic Calogero-Moser Hamiltonian is given by

$$
H_{N}=-\Delta+\beta(\beta-1) \sum_{i \neq j}^{N+1} \wp\left(x_{i}-x_{j}\right) .
$$

Here $\beta$ is a parameter, and $\wp(x)$ is the Weierstrass $\wp$-function with the invariants $g_{2}, g_{3}$, i.e., a solution of the ODE $\wp^{\prime}(x)^{2}=4 \wp(x)^{3}-g_{2} \wp(x)-g_{3}$. In the coordinates

$$
X=\frac{1}{N+1} \sum_{i=1}^{N+1} x_{i}, \quad y_{i}=x_{i}-X .
$$


the operator (3.2) takes the form

$$
H_{N}=-\frac{1}{N+1} \frac{\partial^{2}}{\partial X^{2}}+\mathcal{H}_{N}\left(y_{1}, y_{2}, \ldots y_{N}\right)
$$

where

$$
\mathcal{H}_{N}=-\frac{N}{N+1} \sum_{i=1}^{N} \frac{\partial^{2}}{\partial y_{i}^{2}}+\frac{1}{N+1} \sum_{i \neq j}^{N} \frac{\partial^{2}}{\partial y_{i} \partial y_{j}}+\beta(\beta-1) \sum_{i \neq j}^{N+1} \wp\left(y_{i}-y_{j}\right)
$$

In the last term we have to substitute $-\sum_{i=1}^{N} y_{i}$ for $y_{N+1}$.

In [48] the following transformation $\left(y_{1}, \ldots, y_{N}\right) \rightarrow\left(u_{1}, \ldots, u_{N}\right)$ defined by

$$
\left(\begin{array}{ccccc}
\wp\left(y_{1}\right) & \wp^{\prime}\left(y_{1}\right) & \cdots & \wp^{(N-2)}\left(y_{1}\right) & \wp^{(N-1)}\left(y_{1}\right) \\
\wp\left(y_{2}\right) & \wp^{\prime}\left(y_{2}\right) & \cdots & \wp^{(N-2)}\left(y_{2}\right) & \wp^{(N-1)}\left(y_{2}\right) \\
\vdots & \vdots & \ldots & \vdots & \vdots \\
\wp\left(y_{N}\right) & \wp^{\prime}\left(y_{N}\right) & \cdots & \wp^{(N-2)}\left(y_{N}\right) & \wp^{(N-1)}\left(y_{N}\right)
\end{array}\right)\left(\begin{array}{c}
u_{1} \\
u_{2} \\
\vdots \\
u_{N}
\end{array}\right)=\left(\begin{array}{c}
1 \\
1 \\
\vdots \\
1
\end{array}\right)
$$

was considered. Denote by $D_{N}\left(y_{1}, \ldots, y_{N}\right)$ the Jacobian of the transformation (3.4).

Conjecture 3.1. The gauge transform $\mathcal{H}_{N} \rightarrow D_{N}^{-\frac{\beta}{2}} \mathcal{H}_{N} D_{N}^{\frac{\beta}{2}}$ and subsequent change of variables (3.4) bring (3.3) to a differential operator $P_{N}$ with polynomial coefficients.

In the case $N=2$ the transformation (3.4) coincides with the transformation

$$
u_{1}=\frac{\wp^{\prime}\left(y_{2}\right)-\wp^{\prime}\left(y_{1}\right)}{\wp\left(y_{1}\right) \wp^{\prime}\left(y_{2}\right)-\wp\left(y_{2}\right) \wp^{\prime}\left(y_{1}\right)}, \quad u_{2}=\frac{\wp\left(y_{1}\right)-\wp\left(y_{2}\right)}{\wp\left(y_{1}\right) \wp^{\prime}\left(y_{2}\right)-\wp\left(y_{2}\right) \wp^{\prime}\left(y_{1}\right)},
$$

found in [80]. In addition to explicit form of $P_{2}$, in this paper a polynomial form for the elliptic $G_{2}$-model was found. Polynomial forms for rational and trigonometric Calogero-Moser Hamiltonians in the case of arbitrary $N$ were described in [65].

Remark 3.1. Obviously, for any polynomial form $P$ of Hamiltonian (3.1)

1: the contravariant metric $g$ defining by the symbol of $P$ is flat;

2: $P$ can be reduced to a self-adjoint operator by a gauge transformation $P \rightarrow f P f^{-1}$, where $f$ is a function.

Besides evident properties 1,2 we have in mind the following 
Observation 3.2. (A. Turbiner). For all known integrable cases the polynomial form $P$ preserves some nontrivial finite-dimensional vector space of polynomials.

For Hamiltonians (3.3) the situation can be described as follows. Consider the differential operators $e_{i, j}=E_{i-1, j-1}$, where

$$
\begin{array}{ll}
E_{i j}=y_{i} \frac{\partial}{\partial y_{j}}, & E_{0 i}=\frac{\partial}{\partial y_{i}} \\
E_{00}=-\sum_{j=1}^{N} y_{j} \frac{\partial}{\partial y_{j}}+\beta(N+1), & E_{i 0}=y_{i} E_{00} .
\end{array}
$$

It is easy to verify that they satisfy the commutator relations

$$
e_{i j} e_{k l}-e_{k l} e_{i j}=\delta_{j, k} e_{i l}-\delta_{i, l} e_{k j}, \quad i, j=1, \ldots, N+1
$$

and, therefore, define representations of the Lie algebra $\mathfrak{g l}_{N+1}$ and of the universal enveloping algebra $U\left(\mathfrak{g l}_{N+1}\right)$. The latter representation is not exact.

Conjecture 3.2. The differential operator $P_{N}$ from Conjecture 3.1 can be written as a linear combination of anti-commutators of the operators $E_{i j}$.

The conjectures 3.1 and 3.2 have been verified in [48] for $N=2,3$. Moreover, differential operators with polynomial coefficients that commute with $P_{2}$ and with $P_{3}$ were found. These operators can also be written as non-commutative polynomials in the $E_{i j}$.

Remark 3.2. If $k=-\beta(N+1)$ is a positive integer, then the operators (3.5) preserve the vector space of all polynomials in $y_{1}, \ldots, y_{N}$, whose degrees are not greater than $k$ [65].

\subsubsection{Quasi-solvable differential operators}

Definition 3.1. A linear differential operator

$$
Q=\sum_{i_{1}+\cdots+i_{N} \leq m} a_{i_{1}, \ldots, i_{N}} \partial_{y_{1}}^{i_{1}} \cdots \partial_{y_{N}}^{i_{N}}
$$

of order $m$ with polynomial coefficients is called quasi-solvable if it preserves the vector space of all polynomials in $y_{1}, \ldots, y_{N}$ of degree $\leq k$ for some $k \geq m$.

Theorem 3.1. [72] For any quasi-solvable differential operator (3.7)

$$
\operatorname{deg}\left(a_{i_{1}, \ldots, i_{N}}\right) \leq m+i_{1}+\cdots+i_{N}
$$


Open problem 3.1. Prove that any quasi-solvable operator can be represented as a (non-commutative) polynomial in the variables (3.5), where $k=-\beta(N+1)$.

Remark 3.3. Such a representation is not unique.

\section{ODE case}

Consider the case $N=1$.

Lemma 3.1. Any quasi-solvable operator $P$ of second order has the following structure:

$P=\left(a_{4} x^{4}+a_{3} x^{3}+a_{2} x^{2}+a_{1} x+a_{0}\right) \frac{d^{2}}{d x^{2}}+\left(b_{3} x^{3}+b_{2} x^{2}+b_{1} x+b_{0}\right) \frac{d}{d x}+c_{2} x^{2}+c_{1} x+c_{0}$,

where the coefficients are related by the following identities

$$
b_{3}=2(1-k) a_{4}, \quad c_{2}=k(k-1) a_{4}, \quad c_{1}=k\left(a_{3}-k a_{3}-b_{2}\right) .
$$

The transformation group $\mathrm{GL}_{2}$ acts on the nine-dimensional vector space of such operators as

$$
x \rightarrow \frac{s_{1} x+s_{2}}{s_{3} x+s_{4}}, \quad P \rightarrow\left(s_{3} x+s_{4}\right)^{-k} P\left(s_{3} x+s_{4}\right)^{k} .
$$

The coefficient $a(x)$ of the second derivative is a fourth order polynomial, which transforms as follows

$$
a(x) \rightarrow\left(s_{3} x+s_{4}\right)^{4} a\left(\frac{s_{1} x+s_{2}}{s_{3} x+s_{4}}\right) .
$$

If $a(x)$ has four distinct roots, we call the operator $P$ elliptic. In the elliptic case using transformations (3.8), we may reduce $a$ to

$$
a(x)=4 x(x-1)(x-\kappa),
$$

where $\kappa$ is the elliptic parameter.

Define parameters $n_{1}, \ldots, n_{5}$ by the following identities:

$$
\begin{aligned}
b_{0} & =2\left(1+2 n_{1}\right), \\
b_{1} & =-4\left((\kappa+1)\left(n_{1}+1\right)+\kappa n_{2}+n_{3}\right), \\
b_{2} & =-2\left(3+2 n_{1}+2 n_{2}+2 n_{3}\right), \\
k & =-\frac{1}{2}\left(n_{1}+n_{2}+n_{3}+n_{4}\right), \\
n_{5} & =c_{0}+n_{2}\left(1-n_{2}\right)+\kappa n_{3}\left(1-n_{3}\right)+\left(n_{1}+n_{3}\right)^{2}+\kappa\left(n_{1}+n_{2}\right)^{2} .
\end{aligned}
$$


Then the operator $H=h P h^{-1}$, where

$$
h=x^{\frac{n_{1}}{2}}(x-1)^{\frac{n_{2}}{2}}(x-\kappa)^{\frac{n_{3}}{2}},
$$

has the form

$$
\begin{aligned}
H= & a(x) \frac{d^{2}}{d x^{2}}+\frac{a^{\prime}(x)}{2} \frac{d}{d x}+n_{5}+n_{4}\left(1-n_{4}\right) x+\frac{n_{1}\left(1-n_{1}\right) \kappa}{x} \\
& +\frac{n_{2}\left(1-n_{2}\right)(1-\kappa)}{x-1}+\frac{n_{3}\left(1-n_{3}\right) \kappa(\kappa-1)}{x-\kappa} .
\end{aligned}
$$

Now after the transformation $y=f(x)$, where

$$
f^{\prime 2}=4 f(f-1)(f-\kappa)
$$

we arrive at

$$
\begin{aligned}
H= & \frac{d^{2}}{d y^{2}}+n_{5}+n_{4}\left(1-n_{4}\right) f+\frac{n_{1}\left(1-n_{1}\right) \kappa}{f}+\frac{n_{2}\left(1-n_{2}\right)(1-\kappa)}{f-1} \\
& +\frac{n_{3}\left(1-n_{3}\right) \kappa(\kappa-1)}{f-\kappa} .
\end{aligned}
$$

In general here $n_{i}$ are arbitrary parameters.

Another form of this Hamiltonian (up to a constant) is given by

$$
\begin{aligned}
H= & \frac{d^{2}}{d y^{2}}+n_{4}\left(1-n_{4}\right) \wp(y)+n_{1}\left(1-n_{1}\right) \wp\left(y+\omega_{1}\right)+n_{2}\left(1-n_{2}\right) \wp\left(y+\omega_{2}\right) \\
& +n_{3}\left(1-n_{3}\right) \wp\left(y+\omega_{1}+\omega_{2}\right),
\end{aligned}
$$

where $\omega_{i}$ are half-periods of the Weierstrass function $\wp(x)$. If $n_{1}=n_{2}=$ $n_{3}=0$, we get the Lame operator. In general, it is the Darboux-TreibichVerdier operator [83].

When

$$
k=-\frac{1}{2}\left(n_{1}+n_{2}+n_{3}+n_{4}\right)
$$

is a natural number, this operator $H$ preserves the finite-dimensional vector space of elliptic functions, which corresponds to polynomials for the initial operator $P$.

\section{Two-dimensional operators}

Consider second order differential operators of the form

$$
\begin{array}{r}
P=a(x, y) \frac{\partial^{2}}{\partial x^{2}}+2 b(x, y) \frac{\partial^{2}}{\partial x \partial y}+c(x, y) \frac{\partial^{2}}{\partial y^{2}} \\
+d(x, y) \frac{\partial}{\partial x}+e(x, y) \frac{\partial}{\partial y}+f(x, y)
\end{array}
$$


with polynomial coefficients. Denote by $D(x, y)$ the determinant $a(x, y) c(x, y)-b(x, y)^{2}$. We assume that $D \neq 0$.

Lemma 3.2. The operator (3.9) is quasi-solvable iff the coefficients have the following structure

$$
\begin{aligned}
a= & q_{1} x^{4}+q_{2} x^{3} y+q_{3} x^{2} y^{2}+z_{1} x^{3}+z_{2} x^{2} y+z_{3} x y^{2}+a_{1} x^{2}+a_{2} x y+a_{3} y^{2} \\
& +a_{4} x+a_{5} y+a_{6} ; \\
b= & q_{1} x^{3} y+q_{2} x^{2} y^{2}+q_{3} x y^{3}+\frac{1}{2}\left(z_{4} x^{3}+\left(z_{1}+z_{5}\right) x^{2} y+\left(z_{2}+z_{6}\right) x y^{2}+z_{3} y^{3}\right) \\
& +b_{1} x^{2}+b_{2} x y+b_{3} y^{2}+b_{4} x+b_{5} y+b_{6} ; \\
c= & q_{1} x^{2} y^{2}+q_{2} x y^{3}+q_{3} y^{4}+z_{4} x^{2} y+z_{5} x y^{2}+z_{6} y^{3}+c_{1} x^{2}+c_{2} x y+c_{3} y^{2} \\
& +c_{4} x+c_{5} y+c_{6} ; \\
d= & (1-k)\left(2\left(q_{1} x^{3}+q_{2} x^{2} y+q_{3} x y^{2}\right)+z_{7} x^{2}+\left(z_{2}+z_{8}-z_{6}\right) x y+z_{3} y^{2}\right) \\
& +d_{1} x+d_{2} y+d_{3} ; \\
e= & (1-k)\left(2\left(q_{1} x^{2} y+q_{2} x y^{2}+q_{3} y^{3}\right)+z_{4} x^{2}+\left(z_{5}+z_{7}-z_{1}\right) x y+z_{8} y^{2}\right) \\
& +e_{1} x+e_{2} y+e_{3} ; \\
f= & k(k-1)\left(q_{1} x^{2}+q_{2} x y+q_{3} y^{2}+\left(z_{7}-z_{1}\right) x+\left(z_{8}-z_{6}\right) y\right)+f_{1} .
\end{aligned}
$$

The dimension of the vector space of such operators equals 36 . The group $\mathrm{GL}_{3}$ acts on this vector space in a projective way by transformations

$$
\begin{gathered}
\tilde{x}=\frac{a_{1} x+a_{2} y+a_{3}}{c_{1} x+c_{2} y+c_{3}}, \quad \bar{y}=\frac{b_{1} x+b_{2} y+b_{3}}{c_{1} x+c_{2} y+c_{3}}, \\
\tilde{P}=\left(c_{1} x+c_{2} y+c_{3}\right)^{-k} P \circ\left(c_{1} x+c_{2} y+c_{3}\right)^{k} .
\end{gathered}
$$

This transformation corresponds to the matrix

$$
\left(\begin{array}{ccc}
a_{1} & a_{2} & a_{3} \\
b_{1} & b_{2} & b_{3} \\
c_{1} & c_{2} & c_{3}
\end{array}\right) \in \mathrm{GL}_{3} .
$$

The representation is a sum of irreducible representations $W_{1}, W_{2}$ and $W_{3}$ of dimensions 27, 8 and 1, correspondingly. A basis of $W_{2}$ is given by

$$
\begin{array}{ll}
x_{1}=5 z_{7}-z_{5}-7 z_{1}, & x_{2}=5 z_{8}-z_{2}-7 z_{6}, \\
x_{3}=5 d_{1}+2(k-1)\left(2 a_{1}+b_{2}\right), & x_{4}=5 e_{1}+2(k-1)\left(2 b_{1}+c_{2}\right), \\
x_{5}=5 d_{2}+2(k-1)\left(2 b_{3}+a_{2}\right), & x_{6}=5 e_{2}+2(k-1)\left(2 c_{3}+b_{2}\right), \\
x_{7}=5 d_{3}+2(k-1)\left(a_{4}+b_{5}\right), & x_{8}=5 e_{3}+2(k-1)\left(b_{4}+c_{5}\right) .
\end{array}
$$

The generic orbit of the group action on $W_{2}$ has dimension 6 . There are two polynomial invariants of the action:

$$
I_{1}=x_{3}^{2}-x_{3} x_{6}+x_{6}^{2}+3 x_{4} x_{5}+3(k-1)\left(x_{1} x_{7}+x_{2} x_{8}\right),
$$


and

$$
\begin{gathered}
I_{2}=2 x_{3}^{3}-3 x_{3}^{2} x_{6}-3 x_{3} x_{6}^{2}+2 x_{6}^{3}+9 x_{4} x_{5}\left(x_{3}+x_{6}\right)+ \\
9(k-1)\left(x_{1} x_{3} x_{7}+x_{2} x_{6} x_{8}-2 x_{1} x_{6} x_{7}-2 x_{2} x_{3} x_{8}+3 x_{2} x_{4} x_{7}+3 x_{1} x_{5} x_{8}\right) .
\end{gathered}
$$

\section{Flat polynomial metrics}

According to Remark 3.1, the contravariant metric

$$
g^{1,1}=a, \quad g^{1,2}=g^{2,1}=b, \quad g^{2,2}=c,
$$

defined by the coefficients $a, b, c$ of the operator (3.9) is flat (i.e. $R_{1,2,1,2}=$ 0 ) for any polynomial form $P$.

Open problem 3.2. Describe all flat contravariant metrics defined by the polynomials a, b, c from Lemma 3.2 up to transformations (3.10).

Some particular results were obtained in [72].

Example 3.1. For any constant $\kappa$ the metric $g$ with

$$
\begin{gathered}
a=\left(x^{2}-1\right)\left(x^{2}-\kappa\right)+\left(x^{2}+\kappa\right) y^{2}, \quad b=x y\left(x^{2}+y^{2}+1-2 \kappa\right), \\
c=(\kappa-1)\left(x^{2}-1\right)+\left(x^{2}+2-\kappa\right) y^{2}+y^{4}
\end{gathered}
$$

is flat. Moreover, this is a linear pencil of polynomial contravariant flat metrics with respect to the parameter $\kappa[14]$. The metric is related to a polynomial form [81] for the so called Inozemtsev $B C_{2}$ Hamiltonian

$$
H=\Delta+2 m(m-1)(\wp(x+y)+\wp(x-y))+\sum_{i=0}^{3} n_{i}\left(n_{i}-1\right)\left(\wp\left(x+\omega_{i}\right)+\wp\left(y+\omega_{i}\right)\right),
$$

where $\omega_{0}=0, \omega_{3}=\omega_{1}+\omega_{2}$ and $\omega_{1}, \omega_{2}$ are the half-periods of the Weierstrass function $\wp(x)$.

\subsubsection{Commutative subalgebras in $U\left(\mathfrak{g l}_{N+1}\right)$ and quantum Calogero-Moser Hamiltonians}

A class of commutative subalgebras in $U\left(\mathfrak{g l}_{n}\right)$ was constructed in [86]. These subalgebras are quantizations of commutative Poisson subalgebras generated by compatible constant and linear $\mathfrak{g l}_{n}$-Poisson brackets. The quantization recipe is very simple: any product $\prod_{1}^{k} x_{i}$ of commuting generators should be replaced by $\frac{1}{k !} \sum_{\sigma \in S_{k}} \prod y_{\sigma(i)}$, where $y_{i}$ are noncommutative generators. 
The universal enveloping algebra $U\left(\mathfrak{g l}_{N+1}\right)$ is an associative algebra generated by elements $e_{i j}$ and relations (3.6). Consider the case $N=2$. It turns out that the element of the universal enveloping algebra $U\left(\mathfrak{g l}_{3}\right)$

$$
H=H_{0}+H_{1} g_{2}+H_{2} g_{2}^{2}+H_{3} g_{3},
$$

where

$$
\begin{aligned}
& H_{0}=12 e_{12} e_{11}-12 e_{32} e_{13}-12 e_{33} e_{12}-e_{23}^{2}, \\
& H_{1}=-e_{21}+2 e_{21} e_{11}-e_{22} e_{21}-e_{31} e_{23}-12 e_{32}^{2}-e_{33} e_{21}, \\
& H_{2}=-e_{31}^{2}, \\
& H_{3}=36 e_{32} e_{31}+3 e_{21}^{2},
\end{aligned}
$$

commutes with two third order elements of the form

$$
\begin{aligned}
& K=K_{0}+K_{1} g_{2}+K_{2} g_{3}, \\
& M=M_{0}+M_{1} g_{2}+M_{2} g_{3}+M_{3} g_{2}^{2}+M_{4} g_{2} g_{3}+M_{5} g_{3}^{2}+M_{6} g_{2}^{3} .
\end{aligned}
$$

Here $g_{2}$ and $g_{3}$ are arbitrary parameters and

$$
\begin{aligned}
K_{0}= & -e_{23}+2 e_{21} e_{13}-e_{23} e_{22}-36 e_{32} e_{12}+e_{33} e_{23}-e_{21} e_{13} e_{11} \\
& -e_{22} e_{21} e_{13}+e_{23} e_{11}^{2}+2 e_{23} e_{21} e_{12}-e_{23} e_{22} e_{11}+12 e_{31} e_{12}^{2} \\
& -e_{31} e_{23} e_{13}-12 e_{32} e_{12} e_{11}-e_{32} e_{23}^{2}-12 e_{32}^{2} e_{13}+2 e_{33} e_{21} e_{13} \\
& -e_{33} e_{23} e_{11}+e_{33} e_{23} e_{22}+12 e_{33} e_{32} e_{12} \\
K_{1}= & 3 e_{31} e_{11}-3 e_{31} e_{22}-2 e_{32} e_{21}+e_{31} e_{21} e_{12}+e_{31} e_{22} e_{11} \\
& -e_{31} e_{22}^{2}+e_{31}^{2} e_{13}-2 e_{32} e_{21} e_{11}+e_{32} e_{22} e_{21}-2 e_{32} e_{31} e_{23} \\
& -e_{33} e_{31} e_{11}+e_{33} e_{31} e_{22}+e_{33} e_{32} e_{21}, \\
K_{2}= & 3\left(2 e_{31} e_{21}+e_{31} e_{22} e_{21}+e_{31}^{2} e_{23}-e_{32} e_{21}^{2}-e_{33} e_{31} e_{21}\right) \\
M_{0}=2( & 12 e_{13} e_{11}-6 e_{22} e_{13}-6 e_{33} e_{13}-12 e_{13} e_{11}^{2}-6 e_{22} e_{13} e_{11} \\
& +6 e_{22}^{2} e_{13}+18 e_{23} e_{12} e_{11}-18 e_{23} e_{22} e_{12}+e_{23}^{3}-216 e_{32} e_{12}^{2} \\
& \left.+18 e_{32} e_{23} e_{13}+30 e_{33} e_{13} e_{11}-6 e_{33} e_{22} e_{13}-12 e_{33}^{2} e_{13}\right)
\end{aligned}
$$




$$
\begin{gathered}
M_{1}=-3\left(2 e_{23} e_{21}-36 e_{31} e_{12}+20 e_{32} e_{11}-28 e_{32} e_{22}+8 e_{33} e_{32}-4 e_{23} e_{21} e_{11}\right. \\
+2 e_{23} e_{22} e_{21}-12 e_{31} e_{12} e_{11}-e_{31} e_{23}^{2}+8 e_{32} e_{11}^{2}+36 e_{32} e_{21} e_{12} \\
+4 e_{32} e_{22} e_{11}-4 e_{32} e_{22}^{2}-24 e_{32} e_{31} e_{13}-12 e_{32}^{2} e_{23}+2 e_{33} e_{23} e_{21} \\
\left.+12 e_{33} e_{31} e_{12}-20 e_{33} e_{32} e_{11}+4 e_{33} e_{32} e_{22}+8 e_{33}^{2} e_{32}\right) \\
M_{2}=-18\left(4 e_{31} e_{11}-2 e_{31} e_{22}-2 e_{33} e_{31}-e_{23} e_{21}^{2}-2 e_{31} e_{11}^{2}\right. \\
-6 e_{31} e_{21} e_{12}+2 e_{31} e_{22} e_{11}-2 e_{31} e_{22}^{2}+6 e_{31}^{2} e_{13}+6 e_{32} e_{22} e_{21} \\
\left.+24 e_{32}^{3}+2 e_{33} e_{31} e_{11}+2 e_{33} e_{31} e_{22}-6 e_{33} e_{32} e_{21}-2 e_{33}^{2} e_{31}\right) \\
M_{3}=-3\left(2 e_{31} e_{21}-2 e_{31} e_{21} e_{11}+e_{31} e_{22} e_{21}+e_{31}^{2} e_{23}-24 e_{32}^{2} e_{31}+e_{33} e_{31} e_{21}\right) \\
M_{4}=9\left(e_{31} e_{21}^{2}-12 e_{32} e_{31}^{2}\right), \\
M_{5}=108 e_{31}^{3}, \\
M_{6}=-2 e_{31}^{3} .
\end{gathered}
$$

One can verify that $[K, M]=0$. Thus, we get a commutative subalgebra in $U\left(\mathfrak{g l}_{3}\right)$ generated by the elements $H, K, M$ and by the three central elements of $U\left(\mathfrak{g l}_{3}\right)$ of order 1,2 , and 3 .

This subalgebra generates "integrable" operators ${ }^{1}$ by different representations of $U\left(\mathfrak{g l}_{3}\right)$ by differential, difference and $q$-difference operators.

In particular, the substitution of differential operators (3.5) with two independent variables for $e_{i j}$ maps the element $H$ to a polynomial form $P_{2}$ for the elliptic Calogero-Moser Hamiltonian (3.3) with $N=2$, the element $M$ to a third order differential operator that commutes with $P_{2}$, and the element $K$ to zero. The parameters $g_{2}$ and $g_{3}$ coincide with the invariants of the Weierstrass function $\wp(x)$ from $(3.3)$.

Remark 3.4. The representation of $U\left(\mathfrak{g l}_{3}\right)$ by the matrix unities in $\mathrm{Mat}_{3}$ maps $H, K$ and $M$ to zero.

The representation defined by

$$
e_{i j} \rightarrow z_{i} \frac{\partial}{\partial z_{j}}
$$

\footnotetext{
${ }^{1}$ This means that there exist "rather many" operators commuting with them.
} 
maps $H$ to a homogeneous differential operator with 3 independent variables of the form $\mathcal{H}=\sum_{i \geq j} a_{i j} \frac{\partial^{2}}{\partial z_{i} \partial z_{j}}$, where

$$
\begin{gathered}
a_{11}=-2 g_{2} z_{1} z_{2}-3 g_{3} z_{2}^{2}+g_{2}^{2} z_{3}^{2}, \quad a_{22}=12 g_{2} z_{3}^{2}, \quad a_{33}=z_{2}^{2}, \\
a_{21}=-12 z_{1}^{2}+g_{2} z_{2}^{2}-36 g_{3} z_{3}^{2}, \quad a_{31}=2 g_{2} z_{2} z_{3}, \quad a_{32}=24 z_{1} z_{3},
\end{gathered}
$$

and $M$ to an operator $\mathcal{M}=\sum_{i \geq j \geq k} b_{i j k} \frac{\partial^{3}}{\partial z_{i} \partial z_{j} \partial z_{k}}$ that commutes with $\mathcal{H}$. It is interesting that the lower order terms are absent in both $\mathcal{H}$ and in $\mathcal{M}$.

A similar commutative subalgebra in $U\left(\mathfrak{g l}_{4}\right)$ [48] yields a polynomial form for the elliptic Calogero-Moser Hamiltonian (3.3) with $N=3$

\subsubsection{Bi-Hamiltonian origin of classical elliptic Calogero-Moser models}

Consider the following limit procedure. Any element $f \in U\left(\mathfrak{g l}_{n}\right)$ is a polynomial in the non-commutative variables $e_{i j}$, which satisfy the commutator relation (3.6). Taking all the terms of highest degree in $f$ and replacing there $e_{i j}$ by commutative variables $x_{i j}$, we get a polynomial that we call symbol $(f)$.

It is known that for any elements $f$ and $g$ of $U\left(\mathfrak{g l}_{n}\right)$

$$
\operatorname{symbol}([f, g])=\{\operatorname{symbol}(f), \operatorname{symbol}(g)\},
$$

where $\{$,$\} is the linear Poisson bracket defined by$

$$
\left\{x_{i j}, x_{k l}\right\}=\delta_{j, k} x_{i l}-\delta_{i, l} x_{k j}, \quad i, j=1, \ldots, n,
$$

which corresponds to the Lie algebra $\mathfrak{g l}_{n}$. In particular, if $[f, g]=0$, then

$$
\{\operatorname{symbol}(f), \operatorname{symbol}(g)\}=0 .
$$

Consider polynomials in the commutative variables $x_{i j}$. We will regard $x_{i j}$ as the entries of a matrix $X$. Applying the limit procedure to the generators of the commutative subalgebra in $U\left(\mathfrak{g l}_{3}\right)$ described in Section 3.1.3, we get the polynomials

$$
\begin{gathered}
c_{1}=\operatorname{tr} X, \quad c_{2}=\operatorname{tr} X^{2}, \quad c_{3}=\operatorname{tr} X^{3}, \\
h=h_{0}+h_{1} g_{2}+h_{2} g_{2}^{2}+h_{3} g_{3}, \quad k=k_{0}+k_{1} g_{2}+k_{2} g_{3}, \\
m=m_{0}+m_{1} g_{2}+m_{2} g_{3}+m_{3} g_{2}^{2}+m_{4} g_{2} g_{3}+m_{5} g_{3}^{2}+m_{6} g_{2}^{3},
\end{gathered}
$$


where

$$
\begin{aligned}
& h_{0}=12 x_{12} x_{11}-12 x_{32} x_{13}-12 x_{33} x_{12}-x_{23}^{2}, \\
& h_{1}=2 x_{21} x_{11}-x_{22} x_{21}-x_{31} x_{23}-12 x_{32}^{2}-x_{33} x_{21}, \\
& h_{2}=-x_{31}^{2}, \quad h_{3}=36 x_{32} x_{31}+3 x_{21}^{2}, \\
& k_{0}=-x_{21} x_{13} x_{11}-x_{22} x_{21} x_{13}+x_{23} x_{11}^{2}+2 x_{23} x_{21} x_{12}-x_{23} x_{22} x_{11} \\
& +12 x_{31} x_{12}^{2}-x_{31} x_{23} x_{13}-12 x_{32} x_{12} x_{11}-x_{32} x_{23}^{2}-12 x_{32}^{2} x_{13} \\
& +2 x_{33} x_{21} x_{13}-x_{33} x_{23} x_{11}+x_{33} x_{23} x_{22}+12 x_{33} x_{32} x_{12}, \\
& k_{1}=x_{31} x_{21} x_{12}+x_{31} x_{22} x_{11}-x_{31} x_{22}^{2}+x_{31}^{2} x_{13}-2 x_{32} x_{21} x_{11} \\
& +x_{32} x_{22} x_{21}-2 x_{32} x_{31} x_{23}-x_{33} x_{31} x_{11}+x_{33} x_{31} x_{22}+x_{33} x_{32} x_{21}, \\
& k_{2}=3\left(x_{31} x_{22} x_{21}+x_{31}^{2} x_{23}-x_{32} x_{21}^{2}-x_{33} x_{31} x_{21}\right) ; \\
& m_{0}=2\left(-12 x_{13} x_{11}^{2}-6 x_{22} x_{13} x_{11}+6 x_{22}^{2} x_{13}+18 x_{23} x_{12} x_{11}\right. \\
& -18 x_{23} x_{22} x_{12}+x_{23}^{3}-216 x_{32} x_{12}^{2}+18 x_{32} x_{23} x_{13} \\
& \left.+30 x_{33} x_{13} x_{11}-6 x_{33} x_{22} x_{13}-12 x_{33}^{2} x_{13}\right), \\
& m_{1}=-3\left(-4 x_{23} x_{21} x_{11}+2 x_{23} x_{22} x_{21}-12 x_{31} x_{12} x_{11}-x_{31} x_{23}^{2}+8 x_{32} x_{11}^{2}\right. \\
& +36 x_{32} x_{21} x_{12}+4 x_{32} x_{22} x_{11}-4 x_{32} x_{22}^{2}-24 x_{32} x_{31} x_{13} \\
& -12 x_{32}^{2} x_{23}+2 x_{33} x_{23} x_{21}+12 x_{33} x_{31} x_{12} \\
& \left.-20 x_{33} x_{32} x_{11}+4 x_{33} x_{32} x_{22}+8 x_{33}^{2} x_{32}\right), \\
& m_{2}=-18\left(-x_{23} x_{21}^{2}-2 x_{31} x_{11}^{2}-6 x_{31} x_{21} x_{12}+2 x_{31} x_{22} x_{11}-2 x_{31} x_{22}^{2}\right. \\
& +6 x_{31}^{2} x_{13}+6 x_{32} x_{22} x_{21}+24 x_{32}^{3}+2 x_{33} x_{31} x_{11} \\
& \left.+2 x_{33} x_{31} x_{22}-6 x_{33} x_{32} x_{21}-2 x_{33}^{2} x_{31}\right),
\end{aligned}
$$




$$
\begin{gathered}
m_{3}=-3\left(-2 x_{31} x_{21} x_{11}+x_{31} x_{22} x_{21}+x_{31}^{2} x_{23}-24 x_{32}^{2} x_{31}+x_{33} x_{31} x_{21}\right), \\
m_{4}=9\left(x_{31} x_{21}^{2}-12 x_{32} x_{31}^{2}\right), \quad m_{5}=108 x_{31}^{3}, \quad m_{6}=-2 x_{31}^{3} .
\end{gathered}
$$

These six polynomials commute with each other with respect to the linear $\mathfrak{g l}_{3}$-Poisson bracket (3.11).

It can be verified that elements of the universal enveloping algebra can be reconstructed from the polynomials $(3.12)$ by the quantization procedure described at the beginning of Section 3.1.3.

\section{Quadratic Poisson bracket}

Consider the following quadratic bracket

$$
\{f, g\}_{2}=\{f, g\}_{a}+\kappa\{f, g\}_{b}+\kappa^{2}\{f, g\}_{c},
$$

where $\kappa$ is an arbitrary parameter,

$$
\{f, g\}_{a}=-3 \operatorname{tr}(X)\{f, g\}_{1}, \quad\{f, g\}_{c}=Z_{1}(f) Z_{2}(g)-Z_{1}(g) Z_{2}(f) .
$$

Here the bracket $\{,\}_{1}$ is defined by (3.11). The above vector fields $Z_{i}$ are defined as follows:

$$
Z_{1}(f)=\sum_{i=1}^{3} \frac{\partial f}{\partial x_{i i}}, \quad Z_{2}(f)=\{h, f\}_{1},
$$

and

$$
\{f, g\}_{b}=Z_{3}\left(\{f, g\}_{1}\right)-\left\{Z_{3}(f), g\right\}_{1}-\left\{f, Z_{3}(g)\right\}_{1}
$$

where

$$
Z_{3}(f)=\sum_{i, j=1}^{3} G_{i, j} \frac{\partial f}{\partial x_{i j}} .
$$

Here $\{\cdot, \cdot\}_{1}$ denotes the linear bracket (3.11). The coefficients of the vector field $Z_{3}$ are given by

$$
\begin{gathered}
G_{1,1}=\left(-2 x_{11} x_{23}+x_{22} x_{23}+36 x_{12} x_{32}+x_{23} x_{33}\right) \\
+x_{31}\left(x_{11}-2 x_{22}+x_{33}\right) g_{2}+9 x_{21} x_{31} g_{3}, \\
G_{2,2}=-G_{1,1}, \quad G_{3,3}=0, \\
G_{1,2}=\left(x_{11} x_{13}+x_{13} x_{22}-3 x_{12} x_{23}-2 x_{13} x_{33}\right) \\
+\left(3 x_{12} x_{31}+5 x_{11} x_{32}-4 x_{22} x_{32}-x_{32} x_{33}\right) g_{2} \\
-3\left(2 x_{11} x_{31}-x_{22} x_{31}-3 x_{21} x_{32}-x_{31} x_{33}\right) g_{3},
\end{gathered}
$$




$$
\begin{gathered}
G_{1,3}=3 x_{13} x_{23}-\left(x_{11}-x_{22}\right)\left(x_{11}+x_{22}-2 x_{33}\right) g_{2}-3 x_{21}\left(x_{11}+x_{22}-2 x_{33}\right) g_{3}, \\
G_{2,1}=-3\left(x_{21} x_{23}+12 x_{12} x_{31}+4 x_{11} x_{32}-8 x_{22} x_{32}+4 x_{32} x_{33}\right)-6 x_{21} x_{31} g_{2}, \\
G_{2,3}=3\left(4 x_{11} x_{12}+4 x_{12} x_{22}+x_{23}^{2}-8 x_{12} x_{33}\right)+x_{21}\left(x_{11}+x_{22}-2 x_{33}\right) g_{2}, \\
G_{3,1}=2\left(x_{11} x_{21}+x_{21} x_{22}+18 x_{32}^{2}-2 x_{21} x_{33}\right)-3 x_{31}^{2} g_{2}, \\
G_{3,2}=-\left(x_{11}-x_{22}\right)\left(x_{11}+x_{22}-2 x_{33}\right)+6 x_{31} x_{32} g_{2}-9 x_{31}^{2} g_{3} .
\end{gathered}
$$

Theorem 3.2. i) Formula (3.13) defines a Poisson bracket;

ii) This quadratic bracket is compatible (see Section 1.4) with the linear $\mathfrak{g l}_{3}$-Poisson bracket (3.11);

iii) The Casimir function of the pencil of these two brackets generates (see Theorem 1.1) the commutative Poisson subalgebra described in Section 3.1.4;

Conjecture 3.3. The Poisson bracket (3.13) is the elliptic Poisson bracket of the type $q_{9,2}$ (see [20]) written in an unusual basis.

To get the classical elliptic Calogero-Moser Hamiltonian, one should use the following classical limit of formulas (3.5):

$$
\begin{array}{ll}
x_{i+1, j+1}=q_{i} p_{j}, & x_{1, i+1}=p_{i}, \\
x_{1,1}=-\sum_{j=1}^{N} q_{j} p_{j}+\beta(N+1), & x_{i+1,1}=q_{i} x_{1,1},
\end{array}
$$

where $p_{i}$ and $q_{i}$ are canonical variables for the standard constant Poisson bracket (1.17). One can verify that $p_{i}, q_{i}$ are Darboux coordinates on the minimal symplectic leaf of the $\mathfrak{g l}_{N+1}$-Poisson bracket. This leaf is the orbit of the diagonal matrix $\operatorname{diag}(\beta(\mathrm{N}+1), 0,0, \ldots, 0)$.

In the case $N=2$ after substitution (3.14) into (3.12) we get polynomials in the canonical variables $p_{i}, q_{i}$ commuting with respect to (1.17). The element $h$ becomes the Calogero-Moser Hamiltonian written in unusual coordinates, the element $k$ vanishes, the element $m$ converts to the integral of third degree in momenta that commutes with the Hamiltonian $h$, and the Casimir functions $c_{i}$ become constants. 
To bring the Hamiltonian and the cubic integral to the standard Calogero-Moser form

$$
h_{N}=-\sum_{i=1}^{N+1} p_{i}^{2}+\beta(\beta-1) \sum_{i \neq j}^{N+1} \wp\left(q_{i}-q_{j}\right) .
$$

one has to apply a canonical transformation, where the transformation rule for the coordinates is given by (3.4).

In the case $N=3$ a quadratic bracket exists. This bracket is compatible with the linear $\mathfrak{g l}_{4}$-Poisson bracket and generates the corresponding classical elliptic Calogero-Moser Hamiltonian in the same way as for $N=2$.

Conjecture 3.4. For any $N$ the classical elliptic Calogero-Moser Hamiltonian (3.15) can be obtained from the elliptic Poisson bracket of the $q_{(N+1)^{2}, N}$-type by the above procedure.

Open problem 3.3. For the elliptic bracket $\{$,$\} of the q_{(N+1)^{2}, N}$-type find a basis such that the linear bracket $\{,\}_{1}$ compatible with $\{$,$\} has the$ canonical form (3.11).

\subsection{Bi-Hamiltonian formalism and compati- ble algebras}

\subsubsection{Compatible Lie algebras}

Suppose that two linear finite-dimensional Poisson brackets are compatible. As it was mentioned in the introduction, each of these brackets corresponds to a Lie algebra. Denote by $[\cdot, \cdot]_{1}$ and $[\cdot, \cdot]_{2}$ the operations of these algebras. It is clear that the Poisson brackets are compatible iff the operation $\lambda_{1}[\cdot, \cdot]_{1}+\lambda_{2}[\cdot, \cdot]_{2}$ is a Lie bracket for any $\lambda_{i}$. Without loss of generality we may put $\lambda_{1}=1$.

Definition 3.2. Two Lie brackets $[\cdot, \cdot]$ and $[\cdot, \cdot]_{1}$ defined on the same vector space $\mathbf{V}$ are called compatible if the operation

$$
[\cdot, \cdot]_{\lambda}=[\cdot, \cdot]+\lambda[\cdot, \cdot]_{1}
$$

is a Lie bracket for any $\lambda$.

Suppose that $[\cdot, \cdot]$ corresponds to a semi-simple Lie algebra $\mathcal{G}$. The following classification problem arises:

Open problem 3.4. Describe all possible Lie brackets $[\cdot, \cdot]_{1}$ compatible with a given semi-simple Lie bracket $[\cdot, \cdot]$. 
The Lie algebra with bracket (3.16) can be regarded as a linear deformation of the algebra $\mathcal{G}$. Since any semi-simple Lie algebra is rigid (i.e., the cohomology $H^{2}[\mathcal{G}, \mathcal{G}]$ of the Lie algebra $\mathcal{G}$ vanishes), the bracket (3.16) is isomorphic to $[\cdot, \cdot]$. This means that there exists a formal series of the form

$$
A_{\lambda}=I+R \lambda+S \lambda^{2}+\cdots,
$$

where the coefficients $R, S, \ldots$ are constant linear operators on $\mathcal{G}$ and $I$ is the identity operator, such that

$$
A_{\lambda}^{-1}\left[A_{\lambda}(X), A_{\lambda}(Y)\right]=[X, Y]+\lambda[X, Y]_{1} .
$$

It follows from (3.17) that

$$
[X, Y]_{1}=[R(X), Y]+[X, R(Y)]-R([X, Y])
$$

where $R$ is the corresponding coefficient of $A_{\lambda}$.

Lemma 3.3. The bracket $[\cdot, \cdot]_{1}$ is a Lie bracket iff there exists a linear operator $S: \mathcal{G} \rightarrow \mathcal{G}$ such that

$$
\begin{array}{r}
R([R(X), Y]-[R(Y), X])-[R(X), R(Y)]-R^{2}([X, Y]) \\
=[S(X), Y]-[S(Y), X]-S([X, Y]) .
\end{array}
$$

In the special case $S=0$ the relation from Lemma 3.3 takes the form

$$
R([R(X), Y]-[R(Y), X])-[R(X), R(Y)]-R^{2}([X, Y])=0 .
$$

We present below examples [26] of compatible Lie brackets with the corresponding operators $A_{\lambda}$ and $R$.

Example 3.2. Let $\mathcal{G}$ be the Lie algebra associated with an associative algebra $\mathcal{A}$, then we can take for $R$ the operator of left multiplication by any element $r$. In this case

$$
[X, Y]_{1}=X r Y-Y r X, \quad A_{\lambda}: g \rightarrow g+\lambda r g .
$$

Example 3.3. Let $\mathcal{A}$ be an associative algebra with an involution $*, \mathcal{G}$ be the Lie algebra of all skew-symmetric elements of $\mathcal{A}, r$ be an element symmetric with respect to $*$. In this case the operator

$$
A_{\lambda}: g \rightarrow \sqrt{1+r \lambda} g \sqrt{1+r \lambda}
$$

can be taken as $A_{\lambda}, R(X)=\frac{1}{2}(r X+X r)$ and $[X, Y]_{1}=X r Y-Y r X$.

Example 3.4. Let $\varphi$ be an automorphism of order $n$ of a Lie algebra $\mathcal{G}, \mathcal{G}_{i}$ be the eigenspace of the operator $\varphi$ corresponding to eigenvalue $\varepsilon^{i}$, where $\varepsilon^{n}=1$. Then the vector space $\mathcal{G}_{0}$ is a Lie subalgebra. Suppose that

$$
\mathcal{G}_{0}=\mathcal{G}_{+} \oplus \mathcal{G}_{-}
$$


with vector spaces $\mathcal{G}_{+}$and $\mathcal{G}_{-}$being subalgebras of $\mathcal{G}_{0}$. Consider the operator $A_{\lambda}$ acting on $\mathcal{G}_{+}, \mathcal{G}_{-}$, and $\mathcal{G}_{i}, i>0$, by means of multiplication by

$$
1+\alpha \lambda, \quad 1+\beta \lambda, \quad \sqrt[n]{(1+\alpha \lambda)^{i}(1+\beta \lambda)^{n-i}},
$$

respectively. The operator $R$ is given by

$$
R(g)=\alpha g_{+}+\beta g_{-}+\sum_{i=1}^{n-1}\left(\frac{i}{n} \alpha+\frac{n-i}{n} \beta\right) g_{i}
$$

where $g_{ \pm}$mean the projections of $g$ onto $\mathcal{G}_{ \pm}$.

Example 3.5. This class of compatible brackets is related to finite dimensional $\mathbb{Z}_{2} \times \mathbb{Z}_{2}$-graded Lie algebras. Recall the definition. Let $\varphi$ and $\psi$ be two automorphisms of $\mathcal{G}$ commuting with each other and such that $\varphi^{2}=\psi^{2}=I d$. The decomposition $\mathcal{G}=\oplus \mathcal{G}_{i j}, i, j= \pm 1$ into a direct sum of the following four invariant vector spaces

$$
\mathcal{G}_{i j}=\{g \in \mathcal{G} \mid \varphi(g)=i g, \quad \psi(g)=j g\}
$$

is called the $\mathbb{Z}_{2} \times \mathbb{Z}_{2}$-gradation.

Define an operator $A_{\lambda}$ on the homogeneous components by the formulas

$$
\begin{gathered}
A_{\lambda}\left(g_{1,1}\right)=(1+\gamma \lambda) g_{1,1}, \quad A_{\lambda}\left(g_{-1,1}\right)=\sqrt{(1+\beta \lambda)(1+\gamma \lambda)} g_{-1,1}, \\
A_{\lambda}\left(g_{1,-1}\right)=\sqrt{(1+\alpha \lambda)(1+\gamma \lambda)} g_{1,-1}, \\
A_{\lambda}\left(g_{-1,-1}\right)=\sqrt{(1+\alpha \lambda)(1+\beta \lambda)} g_{-1,-1}+\lambda \sqrt{(\gamma-\alpha)(\gamma-\beta)} \rho\left(g_{-1,-1}\right) .
\end{gathered}
$$

Here $\alpha, \beta$, and $\gamma$ are arbitrary constants and the operator $\rho: \mathcal{G}_{-1,-1} \rightarrow$ $\mathcal{G}_{1,1}$ is any solution of the modified Yang-Baxter equation

$$
\rho([\rho(X), Y]-[\rho(Y), X])-[\rho(X), \rho(Y)]-[X, Y]=0 .
$$

In other words, the Lie algebra $\mathcal{G}_{1,1} \oplus \mathcal{G}_{-1,-1}$ is assumed to be decomposed into a direct sum of $\mathcal{G}_{1,1}$ and some complementary subalgebra $\mathcal{B}$ and $r$ denotes the projection onto $\mathcal{G}_{1,1}$ parallel to $\mathcal{B}$.

The operator $R$ is defined by

$$
\begin{aligned}
R(g)= & \gamma g_{1,1}+\frac{1}{2}(\alpha+\gamma) g_{1,-1}+\frac{1}{2}(\beta+\gamma) g_{-1,1} \\
& +\frac{1}{2}(\alpha+\beta) g_{-1,-1}+\sqrt{(\gamma-\alpha)(\gamma-\beta)} \rho\left(g_{-1,-1}\right)
\end{aligned}
$$

Remark 3.5. The operator $A_{\lambda}$ in Example 3.5 can be parametrized by points of the elliptic curve

$$
\lambda_{1}^{2}-\alpha=\lambda_{2}^{2}-\beta=\lambda_{3}^{2}-\gamma=\frac{1}{\lambda} .
$$


Remark 3.6. A wide class of $\mathbb{Z}_{2} \times \mathbb{Z}_{2}$-graded Lie algebras can be constructed as follows. Let $\mathcal{G}=\oplus \mathcal{G}_{i}$ be $\mathbb{Z}$-graded Lie algebra that possesses an involution $\psi$ such that $\psi\left(\mathcal{G}_{i}\right)=\mathcal{G}_{-i}$. In particular, such an involution exists for any standard gradation of a simple Lie algebra. Taking for $\varphi$ the involution $\varphi(X)=(-1)^{i} X$, where $X \in \mathcal{G}_{i}$, we define on $\mathcal{G}$ a structure of $\mathbb{Z}_{2} \times \mathbb{Z}_{2}$-graded algebra.

\section{Applications}

For applications the operator $A_{\lambda}$ has to be written in a closed form, i.e. as an analytic operator-valued function in $\lambda$. In known examples the $\lambda$ dependence is rational, trigonometric or elliptic.

Very often a Lax pair for the corresponding bi-Hamiltonian model can be written in terms of $A_{\lambda}[28,25]$.

Application 3.1. Consider the following system of ODEs:

$$
w_{t}=[w, v]+w * w, \quad v_{t}=[w, u]+w * v, \quad u_{t}=w * u,
$$

where

$$
X * Y=[R(X), Y]-\left[X, R^{*}(Y)\right]+R^{*}([X, Y]),
$$

and $R^{*}$ stands for the operator adjoint to $R$ with respect to the Killing form. Then the operators

$$
\mathcal{L}=\left(A_{\lambda}^{-1}\right)^{*}\left(\lambda u+v+\lambda^{-1} w\right), \quad \mathcal{A}=\lambda^{-1} A_{\lambda}(w)
$$

form a Lax pair for this system. As usual, the integrals of motion are given by $\operatorname{tr} \mathcal{L}^{k}, \quad k=1,2 \ldots$

In the case of Example 3.2 the first bracket $[\cdot, \cdot]$ is a standard matrix commutator, the second bracket is given by $[x, y]_{1}=x r y-y r x$ and $X * Y=r X Y-Y X r$, where $r$ is an arbitrary matrix. We have

$$
R(w)=r w, \quad A_{\lambda}(w)=(I+\lambda r) w, \quad\left(A_{\lambda}^{-1}\right)^{*}(w)=w(I+\lambda r)^{-1}
$$

If $u=v=0$, then

$$
\mathcal{L}=w(I+\lambda r)^{-1}, \quad \mathcal{A}=\lambda^{-1}(I+\lambda r) w .
$$

The Lax equation is equivalent (up to $t \rightarrow-t$ ) to equation (1.6), where $\mathbf{U}=x, a=r$. For the Lax pair (3.20) we arrive at (2.58), where $u, v$ and $w$ are generic matrices.

Application 3.2. Consider the system of equations

$$
u_{x}=[u, v], \quad v_{y}=[v, u]_{1},
$$

where $u$ and $v$ belong to a vector space $\mathbf{V}$ equipped with two Lie brackets $[\cdot, \cdot]$ and $[\cdot, \cdot]_{1}$. For the well-known integrable principle chiral model

$$
u_{x}=[u, v], \quad v_{y}=[u, v]
$$

the brackets $[\cdot, \cdot]$ and $[\cdot, \cdot]_{1}$ are identical up to sign. 
Theorem 3.3. If the Lie brackets $[\cdot, \cdot]$ and $[\cdot, \cdot]_{1}$ are compatible, then the hyperbolic system (3.21) possesses the following Lax pair

$$
\mathcal{L}=\frac{d}{d y}+\frac{1}{\lambda} A_{\lambda}(u), \quad \mathcal{A}=\frac{d}{d x}+A_{\lambda}(v) .
$$

Compatible Lie algebras and the corresponding operator $A_{\lambda}$ are closely related to different kinds of the Yang-Baxter equation [27, 58].

Application 3.3. Consider the classical Yang-Baxter equation

$$
\left[r^{1,2}(\lambda, \mu), r^{1,3}(\lambda, \nu)\right]+\left[r^{1,2}(\lambda, \mu), r^{2,3}(\mu, \nu)\right]+\left[r^{1,3}(\lambda, \nu), r^{2,3}(\mu, \nu)\right]=0,
$$

where

$$
r(x, y)=\sum_{i} a_{i}(x, y) \otimes b_{i}(x, y)
$$

is a function of two complex variables with values in $\mathfrak{g l}_{N} \otimes \mathfrak{g l}_{N}$ and $r^{i, j}$, where $1 \leq i, j \leq 3, \quad i \neq j$, are the following functions

$$
\begin{aligned}
& r^{1,2}(\lambda, \mu)=\sum_{i} a_{i}(\lambda, \mu) \otimes b_{i}(\lambda, \mu) \otimes 1, \\
& r^{1,3}(\lambda, \nu)=\sum_{i} a_{i}(\lambda, \nu) \otimes 1 \otimes b_{i}(\lambda, \nu), \\
& r^{2,3}(\mu, \nu)=\sum_{i} 1 \otimes a_{i}(\mu, \nu) \otimes b_{i}(\mu, \nu)
\end{aligned}
$$

with values in $\mathfrak{g l}_{N} \otimes \mathfrak{g l}_{N} \otimes \mathfrak{g l}_{N}$. We suppose, as usual, that the unitary condition

$$
r^{1,2}(\lambda, \mu)=-r^{2,1}(\mu, \lambda)
$$

holds, where

$$
r^{2,1}(\mu, \lambda)=\sum_{i} b_{i}(\mu, \lambda) \otimes a_{i}(\mu, \lambda) \otimes 1 .
$$

Theorem 3.4. Let $[\cdot, \cdot]_{1}$ and $[\cdot, \cdot]_{2}$ be two compatible Lie brackets on a $N$ dimensional vector space V. Suppose that there exists a non-degenerate symmetric form $\omega(X, Y)$ on $\mathbf{V}$ invariant with respect to both brackets $[\cdot, \cdot]_{1,2}$. Let $\mathbf{e}_{\mathbf{1}}, \ldots, \mathbf{e}_{\mathbf{N}}$ be a basis orthonormalized with respect to $\omega$. Then

$$
r(x, y)=\sum_{i=1}^{N} \frac{\left(x a d_{1} \mathbf{e}_{\mathbf{i}}+a d_{2} \mathbf{e}_{\mathbf{i}}\right) \otimes\left(y a d_{1} \mathbf{e}_{\mathbf{i}}+a d_{2} \mathbf{e}_{\mathbf{i}}\right)}{x-y}
$$

satisfies the classical Yang-Baxter equation. Here $a d_{i} q$ are linear operators defined by

$$
\operatorname{ad}_{i} q(p)=[q, p]_{i}, \quad i=1,2 .
$$


Application 3.4. The operator form of the classical Yang-Baxter equation [68] is given by

$$
[r(u, w) x, r(u, v) y]=r(u, v)[r(v, w) x, y]+r(u, w)[x, r(w, v) y] .
$$

Here $r(u, v) \in \operatorname{End}(\mathcal{G})$. The solution is called unitary if $\langle x, r(u, v) y\rangle=$ $-\langle r(v, u) x, y\rangle$ for the Killing form of $\mathcal{G}$.

Theorem 3.5. If $A_{\lambda}$ satisfies (3.17); then

$$
r(u, v)=\frac{1}{u-v} A_{u} A_{v}^{-1}
$$

satisfies the Yang-Baxter equation.

Remark 3.7. The $r$-matrix (3.22) is unitary with respect to the form $\langle\cdot, \cdot\rangle$ if the operator $A_{\lambda}$ is orthogonal. In this case the formula (3.17) implies that the form $\langle\cdot, \cdot\rangle$ is invariant also with respect to the bracket $[\cdot, \cdot]_{1}$.

Application 3.5. It is known that decompositions (2.40) of the loop algebra over a Lie algebra $\mathcal{G}$ into a sum of the Lie algebra of all Taylor series and a factoring subalgebra $\mathcal{U}$ gives rise to Lax representations for diverse integrable models (see Section 2.3).

A factoring subalgebra $\mathcal{U}$ is said to be homogeneous if it has the multiplicand $\lambda^{-1}$. This means that

$$
\frac{1}{\lambda} \mathcal{U} \subset \mathcal{U}
$$

It turns out [25] that for any semi-simple Lie algebra $\mathcal{G}$ with a bracket $[\cdot, \cdot]$ there exists a one-to-one correspondence between the homogeneous subalgebras and brackets $[\cdot, \cdot]_{1}$ compatible with $[\cdot, \cdot]$.

\subsubsection{Compatible associative algebras}

While Problem 3.4 for Lie algebras seems to be very difficult, a similar problem for associative algebras is more treatable [58, 57, 55].

Definition 3.3. Two associative algebras with multiplications $\star$ and $\circ$ defined on the same finite dimensional vector space $\mathbf{V}$ are said to be compatible if the multiplication

$$
a \bullet b=a \star b+\lambda a \circ b
$$

is associative for any constant $\lambda$.

Remark 3.8. For compatible associative algebras with multiplications $\star$ and $\circ$ the Lie algebras with the brackets $[X, Y]_{1}=X \star Y-Y \star X$ and $[X, Y]_{2}=X \circ Y-Y \circ X$ are compatible. 
Example 3.6. Let $\mathbf{V}$ be the vector space of polynomials of degree $\leq k-1$ in one variable, $\mu_{1}$ and $\mu_{2}$ be polynomials of degree $k$ without common roots. Any polynomial $Z$, where $\operatorname{deg} Z \leq 2 k-1$, can be uniquely represented in the form $Z=\mu_{1} P+\mu_{2} Q$, where $P, Q \in \mathbf{V}$. Define multiplications $\circ$ and $\star$ on $\mathbf{V}$ by the formula

$$
X Y=\mu_{1}(X \circ Y)+\mu_{2}(X \star Y), \quad X, Y \in \mathbf{V} .
$$

It can be verified that associative algebras with products $\circ$ and $\star$ are compatible.

Example 3.7. Let $\mathbf{e}_{1}, \ldots, \mathbf{e}_{m}$ be a basis of $\mathbf{V}$ and let the multiplication $\star$ be given by

$$
\mathbf{e}_{i} \star \mathbf{e}_{j}=\delta_{j}^{i} \mathbf{e}_{i}
$$

Let

$$
r_{i i}=q_{0}-\sum_{k \neq i} r_{k i}, \quad r_{i j}=\frac{q_{i} \lambda_{i}}{\lambda_{j}-\lambda_{i}}, \quad i \neq j,
$$

where $\lambda_{i}, q_{j}$ are arbitrary constants. Then the product defined by the formula

$$
\mathbf{e}_{i} \circ \mathbf{e}_{j}=r_{i j} \mathbf{e}_{j}+r_{j i} \mathbf{e}_{i}-\delta_{j}^{i} \sum_{k=1}^{m} r_{i k} \mathbf{e}_{k}
$$

is associative and compatible with $\star$. Since this product is linear with respect to the parameters $q_{i}$, we have constructed a family of $m+1$ pairwise compatible associative multiplications.

Suppose that the associative algebra $A$ with multiplication $\star$ is semisimple. Since such algebras are rigid, the associative algebra with the multiplication $\bullet$ is isomorphic to the algebra $A$ for almost all values of the parameter $\lambda$. Hence there exists a linear operator $S_{\lambda}$ on $\mathbf{V}$ such that

$$
S_{\lambda}(X) \star S_{\lambda}(Y)=S_{\lambda}(X \star Y+\lambda X \circ Y) .
$$

If

$$
S_{\lambda}=\mathbf{1}+R \lambda+O\left(\lambda^{2}\right)
$$

then the multiplication $\circ$ is given by

$$
X \circ Y=R(X) \star Y+X \star R(Y)-R(X \star Y) .
$$

Consider the case when the associative algebra with multiplication $\star$ coincides with $\mathrm{Mat}_{m}$. Then $R: \mathrm{Mat}_{m} \rightarrow \mathrm{Mat}_{m}$ is a linear operator on the space of $m \times m$-matrices. We will omit the sign $\star$. In other words, we investigate associative linear deformations of the matrix product. 
Example 3.8. (see Example 3.2). Let $c$ be an element of $\mathrm{Mat}_{m}$ and $R: X \rightarrow c X$ be the operator of left multiplication by $c$. Then the corresponding multiplication $X \circ Y=X c Y$ is associative and compatible with the standard matrix product in $\mathrm{Mat}_{m}$.

Example 3.9. Suppose that $a, b \in \mathrm{Mat}_{2}$; then the product

$$
X \circ Y=(a X-X a)(b Y-Y b)
$$

is compatible with the standard product in Mat $_{2}$. The corresponding operator $R$ is given by

$$
R(X)=a(X b-b X)
$$

Proposition 3.1. In the case of $\mathrm{Mat}_{2}$ any linear deformation of the matrix product is given by one of these two examples.

Remark 3.9. If $a, b \in \mathrm{Mat}_{m}, m>2$, we need the additional assumption $a^{2}=b^{2}=1$ for (3.24) to be compatible with the product in $\mathrm{Mat}_{m}$.

In the matrix case the operator $R$ is defined up to the transformation

$$
R \rightarrow R+\operatorname{ad}_{s}
$$

where $s \in$ Mat $_{m}$. For any $s$ this transformation does not change the multiplication (3.23).

It is easy to see that any operator $R:$ Mat $_{m} \rightarrow$ Mat $_{m}$ can be written in the form

$$
R(x)=\mathbf{a}_{1} x \mathbf{b}^{1}+\ldots+\mathbf{a}_{p+1} x \mathbf{b}^{p+1},
$$

where $\mathbf{a}_{i}, \mathbf{b}^{i} \in$ Mat $_{m}$. We will assume that $p$ is as small as possible. In this case the matrices $\mathbf{a}_{1}, \ldots, \mathbf{a}_{p+1}$ as well as $\mathbf{b}^{1}, \ldots, \mathbf{b}^{p+1}$ are linearly independent. Using (3.25), we can represent $R(x)$ in the following form:

$$
R(x)=\mathbf{a}_{1} x \mathbf{b}^{1}+\ldots+\mathbf{a}_{p} x \mathbf{b}^{p}+\mathbf{c} x .
$$

\section{Integrable matrix ODEs related to $R$-operator}

Let $R:$ Mat $_{m} \rightarrow$ Mat $_{m}$ be a linear operator such that the product (3.23) is associative. Consider $[28,57]$ the following matrix differential equation:

$$
\frac{d x}{d t}=\left[x, R(x)+R^{*}(x)\right], \quad x(t) \in \mathrm{Mat}_{m},
$$

and $R^{*}$ stands for the operator adjoint to $R$ with respect to the bi-linear form $\langle x, y\rangle=\operatorname{tr}(x y)$. For an operator written in the form (3.26) we have

$$
R^{*}(x)=\mathbf{b}^{1} x \mathbf{a}_{1}+\ldots+\mathbf{b}^{p} x \mathbf{a}_{p}+x \mathbf{c} .
$$


Theorem 3.6. Equation (3.27) possesses the following Lax pair:

$$
L=\left(S_{\lambda}^{-1}\right)^{*}(x), \quad A=\frac{1}{\lambda} S_{\lambda}(x) .
$$

Open problem 3.5. Show that equation (3.27) is bi-Hamiltonian with the Hamiltonian operators

$$
\mathcal{H}_{1}=a d_{x}, \quad \mathcal{H}_{2}=a d_{x}^{1},
$$

where $a d_{x}^{1}$ is defined by the multiplication (3.23).

Example 3.10. In the case of Example 3.8 we get

$$
\frac{d x}{d t}=[x, x c+c x]=x^{2} c-c x^{2}
$$

for $m \times m$-matrix $x$ and any constant matrix $c$. Under the reductions $x^{T}=-x, c^{T}=c$ the equation is a generalized symmetry for the $n$ dimensional Euler top.

Example 3.11. In the case of Remark 3.9 we have [57]

$$
x_{t}=[x, b x a+a x b+x b a+b a x], \quad x, a, b \in \mathrm{Mat}_{m},
$$

where $a^{2}=b^{2}=\mathbf{1}_{m}$. Equation (3.28) admits the following skewsymmetric reduction

$$
x^{T}=-x, \quad b=a^{T} .
$$

Different integrable $\mathfrak{s o}_{m}$-models provided by this reduction are in one-toone correspondence with equivalence classes of $m \times m$ matrices $a$ such that $a^{2}=1$ with respect to the $S O_{m}$ gauge action. For the real matrix $a$, a canonical form for such equivalence class can be chosen as

$$
a=\left(\begin{array}{cc}
\mathbf{1}_{p} & T \\
0 & \mathbf{1}_{m-p}
\end{array}\right)
$$

Here $\mathbf{1}_{s}$ stands for the unity $s \times s$-matrix and $T=\left\{t_{i j}\right\}$, where $t_{i j}=\delta_{i j} \alpha_{i}$. This canonical form is defined by the discrete natural parameter $p$ and continuous parameters $\alpha_{1}, \ldots, \alpha_{r}$, where $p \leq m / 2, r=\min (p, m-p)$.

For example, in the case $m=4$ the equivalence classes with $p=2$ and $p=1$ give rise to the $\mathfrak{s o}_{4}$ Steklov and Poincaré integrable models, respectively.

Thus, whereas Example 3.10 leads to the matrix version of the $\mathfrak{s o}_{4}$ Schottky-Manakov top, the tops corresponding to Example 3.11 with $p=[m / 2]$ and $p=1$ can be regarded as generalizations for the $\mathfrak{s o}_{4}$ Steklov and Poincaré models.

For Example 3.8 the operator $R$ is given by $R(x)=c x$. In the case of Remark 3.9 we have $R(x)=a x b+b a x$. Both of the $R$-operators are written in the form (3.26).

Several classes of integrable equations (3.27) can be constructed using results of the next section. 


\section{Algebraic structure associated with $R$-operator}

Consider an operator $R$ of the form (3.26) that defines an associative product (3.23). Our aim is to study $[58,55]$ the structure of the associative algebra $\mathcal{M}$ generated by $\mathbf{a}_{i}, \mathbf{b}^{i}, \mathbf{c}$ and $\mathbf{1}$. Denote by $\mathcal{L}$ the $2 p+2$ dimensional vector space spanned by these matrices.

Lemma 3.4. If the product (3.23) is associative, then

$$
\mathbf{a}_{i} \mathbf{a}_{j}=\sum_{k} \phi_{i, j}^{k} \mathbf{a}_{k}+\mu_{i, j} \mathbf{1}, \quad \mathbf{b}^{i} \mathbf{b}^{j}=\sum_{k} \psi_{k}^{i, j} \mathbf{b}^{k}+\lambda^{i, j} \mathbf{1}
$$

for some tensors $\phi_{i, j}^{k}, \mu_{i, j}, \psi_{k}^{i, j}, \lambda^{i, j}$.

This means that the vector spaces spanned by $\mathbf{1}, \mathbf{a}_{1}, \ldots \mathbf{a}_{p}$ and $\mathbf{1}, \mathbf{b}^{1}, \ldots \mathbf{b}^{p}$ are associative algebras. We denote them by $\mathcal{A}$ and $\mathcal{B}$, respectively. These algebras should be in some sense compatible with each other. The simplest example of such compatibility can be described as follows.

Example 3.12. Let $\mathcal{A}$ and $\mathcal{B}$ be associative algebras with basis $A_{1}, \ldots, A_{p}$ and $B^{1}, \ldots, B^{p}$ and structural constants $\phi_{j, k}^{i}$ and $\psi_{\gamma}^{\alpha, \beta}$. Suppose that the structural constants satisfy the following identities:

$$
\phi_{j, k}^{s} \psi_{s}^{l, i}=\phi_{s, k}^{l} \psi_{j}^{s, i}+\phi_{j, s}^{i} \psi_{k}^{l, s}, \quad 1 \leq i, j, k, l \leq p .
$$

Here and below we assume that the summation is carried out over repeated indices. Then the algebra $\mathcal{M}$ of dimension $2 p+p^{2}$ with the basis $A_{i}, B^{j}, A_{i} B^{j}$ and relations

$$
B^{i} A_{j}=\psi_{j}^{k, i} A_{k}+\phi_{j, k}^{i} B^{k}
$$

is associative. This structure is called an associative bi-algebra [3].

In general case the compatibility of $\mathcal{A}$ and $\mathcal{B}$ is described by

Lemma 3.5. If (3.23) is associative, then

$$
\phi_{j, k}^{s} \psi_{s}^{l, i}=\phi_{s, k}^{l} \psi_{j}^{s, i}+\phi_{j, s}^{i} \psi_{k}^{l, s}+\delta_{k}^{l} t_{j}^{i}-\delta_{j}^{i} t_{k}^{l}-\delta_{j}^{i} \phi_{s, r}^{l} \psi_{k}^{r, s},
$$

and

$$
\mathbf{b}^{i} \mathbf{a}_{j}=\psi_{j}^{k, i} \mathbf{a}_{k}+\phi_{j, k}^{i} \mathbf{b}^{k}+t_{j}^{i} \mathbf{1}+\delta_{j}^{i} \mathbf{c},
$$

for some tensor $t_{j}^{i}$.

The remaining matrix c obeys the following relations:

Lemma 3.6. If (3.23) is associative, then

$$
\mathbf{b}^{i} \mathbf{c}=\lambda^{k, i} \mathbf{a}_{k}-t_{k}^{i} \mathbf{b}^{k}-\phi_{k, l}^{i} \psi_{s}^{l, k} \mathbf{b}^{s}-\phi_{k, l}^{i} \lambda^{l, k} \mathbf{1},
$$




$$
\mathbf{c} \mathbf{a}_{j}=\mu_{j, k} \mathbf{b}^{k}-t_{j}^{k} \mathbf{a}_{k}-\phi_{k, l}^{s} \psi_{j}^{l, k} \mathbf{a}_{s}-\mu_{k, l} \psi_{j}^{l, k} \mathbf{1}
$$

where

$\phi_{j, k}^{s} t_{s}^{i}=\psi_{j}^{s, i} \mu_{s, k}+\phi_{j, s}^{i} t_{k}^{s}-\delta_{j}^{i} \psi_{k}^{s, r} \mu_{r, s}, \quad \psi_{s}^{k, i} t_{j}^{s}=\phi_{j, s}^{i} \lambda^{k, s}+\psi_{j}^{s, i} t_{s}^{k}-\delta_{j}^{i} \phi_{s, r}^{k} \lambda^{r, s}$.

Relations (3.29)-(3.31) mean that the vector space $\mathcal{L}$ is a left $\mathcal{B}$-module and a right $\mathcal{A}$-module.

\section{Invariant description}

In this section we forget that the generators of $\mathcal{L}$ are matrices and give a purely algebraic description of the structure appeared above.

Definition 3.4. By weak $\mathcal{M}$-structure on a linear space $\mathcal{L}$ we mean a collection of the following data:

- Two subspaces $\mathcal{A} \subset \mathcal{L}$ and $\mathcal{B} \subset \mathcal{L}$ and a distinguished element $1 \in \mathcal{A} \cap \mathcal{B}$.

- A non-degenerate symmetric scalar product $(\cdot, \cdot)$ on the space $\mathcal{L}$.

- Two associative products $\mathcal{A} \times \mathcal{A} \rightarrow \mathcal{A}$ and $\mathcal{B} \times \mathcal{B} \rightarrow \mathcal{B}$ with a unity 1.

- A left action $\mathcal{B} \times \mathcal{L} \rightarrow \mathcal{L}$ of the algebra $\mathcal{B}$ and a right action $\mathcal{L} \times \mathcal{A} \rightarrow \mathcal{L}$ of the algebra $\mathcal{A}$ on the space $\mathcal{L}$, which commute with each other.

This data should satisfy the following properties:

1. $\operatorname{dim} \mathcal{A} \cap \mathcal{B}=\operatorname{dim} \mathcal{L} /(\mathcal{A}+\mathcal{B})=1$. The intersection of $\mathcal{A}$ and $\mathcal{B}$ is a one dimensional space spanned by the unity 1 .

2. The restriction of the action $\mathcal{B} \times \mathcal{L} \rightarrow \mathcal{L}$ to the subspace $\mathcal{B} \subset \mathcal{L}$ is the product in $\mathcal{B}$. The restriction of the action $\mathcal{L} \times \mathcal{A} \rightarrow \mathcal{L}$ to the subspace $\mathcal{A} \subset \mathcal{L}$ is the product in $\mathcal{A}$.

3. $\left(A_{1}, A_{2}\right)=\left(B^{1}, B^{2}\right)=0$ and

$$
\left(B^{1} B^{2}, v\right)=\left(B^{1}, B^{2} v\right), \quad\left(v, A_{1} A_{2}\right)=\left(v A_{1}, A_{2}\right)
$$

for any $A_{1}, A_{2} \in \mathcal{A}, B^{1}, B^{2} \in \mathcal{B}$ and $v \in \mathcal{L}$.

Remark 3.10. It follows from these properties that $(\cdot, \cdot)$ gives a nondegenerate pairing between the quotient spaces $\mathcal{A} / \mathbb{C}_{1}$ and $\mathcal{B} / \mathbb{C}_{1}$, so $\operatorname{dim} \mathcal{A}=\operatorname{dim} \mathcal{B}$ and $\operatorname{dim} \mathcal{L}=2 \operatorname{dim} \mathcal{A}$.

For a given weak $\mathcal{M}$-structure we can define an algebra generated by $\mathcal{L}$ with natural compatibility and universality conditions. 
Definition 3.5. By weak $\mathcal{M}$-algebra associated with a weak $\mathcal{M}$-structure on $\mathcal{L}$ we mean an associative algebra $U(\mathcal{L})$ with the following properties:

1. $\mathcal{L} \subset \mathcal{U}(\mathcal{L})$ and the actions $\mathcal{B} \times \mathcal{L} \rightarrow \mathcal{L}, \quad \mathcal{L} \times \mathcal{A} \rightarrow \mathcal{L}$ are restrictions of the product in $U(\mathcal{L})$.

2. For any algebra $X$ with the property 1 there exists a unique homomorphism of algebras $X \rightarrow U(\mathcal{L})$ that is identical on $\mathcal{L}$.

\section{Explicit formulas for $U(\mathcal{L})$}

Let $\left\{\bar{A}_{1}, \ldots, \bar{A}_{p}\right\}$ be a basis of $\mathcal{A} / \mathbb{C}_{1}$ and $\left\{\bar{B}^{1}, \ldots, \bar{B}^{p}\right\}$ be a dual basis of $\mathcal{B} / \mathbb{C}_{1}$. This means that $\left(\bar{A}_{i}, \bar{B}^{j}\right)=\delta_{i}^{j}$. It is clear, that $1, A_{1}, \ldots, A_{p}$ and $1, B^{1}, \ldots B^{p}$, where $A_{i} \in \bar{A}_{i}, B_{i} \in \bar{B}_{i}$, are bases in $\mathcal{A}$ and $\mathcal{B}$, respectively. The element $C \in \mathcal{L}$ does not belong to the sum of $\mathcal{A}$ and $\mathcal{B}$. Since $(\cdot, \cdot)$ is non-degenerate, we have $(1, C) \neq 0$. Without loss of generality we may assume that $(1, C)=1,(C, C)=\left(C, A_{i}\right)=\left(C, B^{j}\right)=0$. Given basis of $\mathcal{A}$ and $\mathcal{B}$, such an element $C$ is uniquely determined.

Proposition 3.2. The algebra $U(\mathcal{L})$ is defined by the following relations

$$
\begin{gathered}
A_{i} A_{j}=\phi_{i, j}^{k} A_{k}+\mu_{i, j} 1, \quad B^{i} B^{j}=\psi_{k}^{i, j} B^{k}+\lambda^{i, j} 1 \\
B^{i} A_{j}=\psi_{j}^{k, i} A_{k}+\phi_{j, k}^{i} B^{k}+t_{j}^{i} 1+\delta_{j}^{i} C, \\
B^{i} C=\lambda^{k, i} A_{k}+u_{k}^{i} B^{k}+p^{i} 1, \quad C A_{j}=\mu_{j, k} B^{k}+u_{j}^{k} A_{k}+q_{i} 1
\end{gathered}
$$

for certain tensors $\phi_{i, j}^{k}, \psi_{k}^{i, j}, \mu_{i, j}, \lambda^{i, j}, u_{k}^{i}, p^{i}, q_{i}$.

Let us define an element $K \in U(\mathcal{L})$ by the formula

$$
K=A_{i} B^{i}+C .
$$

Definition 3.6. A weak $\mathcal{M}$-structure on $\mathcal{L}$ is called $\mathcal{M}$-structure if $K \in$ $U(\mathcal{L})$ is a central element of the algebra $U(\mathcal{L})$.

Theorem 3.7. (cf. Example 3.12). For any $\mathcal{M}$-structure the algebra $U(\mathcal{L})$ is spanned by the elements $K^{s}, A_{i} K^{s}, B_{j} K^{s}, A_{i} B^{j} K^{s}$, where $i, j=$ $1, \ldots, p$, and $s=0,1,2, \ldots$

Theorem 3.8. For any representation $U(\mathcal{L}) \rightarrow$ Mat $_{m}$ given by

$$
A_{1} \rightarrow \mathbf{a}_{1}, \ldots, A_{p} \rightarrow \mathbf{a}_{p}, \quad B^{1} \rightarrow \mathbf{b}^{1}, \ldots, B^{p} \rightarrow \mathbf{b}^{p}, \quad C \rightarrow \mathbf{c}
$$

the formula

$$
X \circ Y=R(X) Y+X R(Y)-R(X Y)
$$

where

$$
R(x)=\mathbf{a}_{1} x \mathbf{b}^{1}+\ldots+\mathbf{a}_{p} x \mathbf{b}^{p}+\mathbf{c} x,
$$

defines an associative product on $\mathrm{Mat}_{m}$ compatible with the usual matrix product. 
Example 3.13. Suppose that $\mathcal{A}$ and $\mathcal{B}$ are generated by elements $A \in \mathcal{A}$ and $B \in \mathcal{B}$ such that $A^{p+1}=B^{p+1}=1$. Assume that $\left(B^{i}, A^{-i}\right)=\epsilon^{i}-1$, $(1, C)=1$ and other scalar products are equal to zero. Here $\epsilon$ is a primitive root of unity of order $p$. Let

$$
B^{i} A^{j}=\frac{\epsilon^{-j}-1}{\epsilon^{-i-j}-1} A^{i+j}+\frac{\epsilon^{i}-1}{\epsilon^{i+j}-1} B^{i+j}
$$

for $i+j \neq 0$ modulo $p$ and

$$
\begin{gathered}
B^{i} A^{-i}=1+\left(\epsilon^{i}-1\right) C, \\
C A^{i}=\frac{1}{1-\epsilon^{i}} A^{i}+\frac{1}{\epsilon^{i}-1} B^{i}, \\
B^{i} C=\frac{1}{\epsilon^{-i}-1} A^{i}+\frac{1}{1-\epsilon^{-i}} B^{i}
\end{gathered}
$$

for $i \neq 0$ modulo $p$. These formulas define an $\mathcal{M}$-structure.

The central element has the following form

$$
K=C+\sum_{0<i<p} \frac{1}{\epsilon^{i}-1} A^{-i} B^{i} .
$$

Let $a, t$ be linear operators on some vector space. Assume that $a^{p+1}=1$, $a t=\epsilon t a$ and that the operator $t-1$ is invertible. It is easy to check that the formulas

$$
A \rightarrow a, \quad B \rightarrow \frac{\epsilon t-1}{t-1} a, \quad C \rightarrow \frac{t}{t-1}
$$

define a representation of the algebra $U(\mathcal{L})$.

\section{Case of semi-simple $\mathcal{A}$ and $\mathcal{B}$}

Proposition 3.3. Suppose that for a weak $\mathcal{M}$-structure the algebra $\mathcal{A}$ is semi-simple:

$$
\mathcal{A}=\oplus_{1 \leq i \leq r} \operatorname{End}\left(V_{i}\right), \quad \operatorname{dim} V_{i}=m_{i} .
$$

Then $\mathcal{L}$ as a right $\mathcal{A}$-module is isomorphic to $\oplus_{1 \leq i \leq r}\left(V_{i}^{*}\right)^{2 m_{i}}$.

Proof. Since any right $\mathcal{A}$-module has the form $\oplus_{1 \leq i \leq r}\left(V_{i}^{*}\right)^{l_{i}}$ for some $l_{1}, \ldots, \quad l_{r} \geq 0$, we have $\mathcal{L}=\oplus_{1 \leq i \leq r} \mathcal{L}_{i}$, where $\mathcal{L}_{i}=\left(V_{i}^{*}\right)^{l_{i}}$. Note that $\mathcal{A} \subset \mathcal{L}$ and, moreover, $\operatorname{End}\left(V_{i}\right) \subset \mathcal{L}_{i}$ for $i=1, \ldots, r$. Besides, $\operatorname{End}\left(V_{i}\right) \perp$ $\mathcal{L}_{j}$ for $i \neq j$. Indeed, we have $(v, a)=\left(v, I d_{i} a\right)=\left(v I d_{i}, a\right)=0$ for $v \in \mathcal{L}_{j}$ and $a \in \operatorname{End}\left(V_{i}\right)$, where $I d_{i}$ is the unity of the subalgebra $\operatorname{End}\left(V_{i}\right)$. Since $(\cdot, \cdot)$ is non-degenerate and $\operatorname{End}\left(V_{i}\right) \perp \operatorname{End}\left(V_{i}\right)$ by Property 3 of weak $\mathcal{M}$-structure, we have $\operatorname{dim} \mathcal{L}_{i} \geq 2 \operatorname{dim} \operatorname{End}\left(V_{i}\right) . \operatorname{But} \sum_{i} \operatorname{dim} \mathcal{L}_{i}=\operatorname{dim} \mathcal{L}=$ $2 \operatorname{dim} \mathcal{A}=\sum_{i} 2 \operatorname{dim} \operatorname{End}\left(V_{i}\right)$ and we obtain $\operatorname{dim} \mathcal{L}_{i}=2 \operatorname{dim} \operatorname{End}\left(V_{i}\right)$ for each $i=1, \ldots, r$ which is equivalent to the statement of the proposition. 
Theorem 3.9. Suppose that a vector space $\mathcal{L}$ is equipped with a weak $\mathcal{M}$-structure such that the associative algebras $\mathcal{A}$ and $\mathcal{B}$ are semi-simple:

$$
\begin{array}{cc}
\mathcal{A}=\oplus_{1 \leq i \leq r} \operatorname{End}\left(V_{i}\right), & \mathcal{B}=\oplus_{1 \leq j \leq s} \operatorname{End}\left(W_{j}\right), \\
\operatorname{dim} V_{i}=m_{i}, & \operatorname{dim} W_{j}=n_{j} .
\end{array}
$$

Then $\mathcal{L}$ as an $\mathcal{A} \otimes \mathcal{B}$-module is given by the formula

$$
\mathcal{L}=\oplus_{1 \leq i \leq r, 1 \leq j \leq s}\left(V_{i}^{\star} \otimes W_{j}\right)^{a_{i, j}}
$$

for some integers $a_{i, j} \geq 0$, and

$$
\sum_{j=1}^{s} a_{i, j} n_{j}=2 m_{i}, \quad \sum_{i=1}^{r} a_{i, j} m_{i}=2 n_{j} .
$$

Proof. It is known that any $\mathcal{A} \otimes \mathcal{B}$-module has the form (3.33). Applying Proposition 3.3, we obtain $\operatorname{dim} \mathcal{L}_{i}=2 m_{i}^{2}$, where $\mathcal{L}_{i}=\oplus_{1 \leq j \leq s}\left(V_{i}^{*} \otimes W_{j}\right)^{a_{i, j}}$ - This gives the first equation from (3.34). The second equation can be obtained similarly.

Remark 3.11. Since the dimensions of $\mathcal{A}$ and $\mathcal{B}$ coincide we have

$$
\sum_{i=1}^{r} m_{i}^{2}=\sum_{i=1}^{s} n_{i}^{2}
$$

Definition 3.7. The $r \times s$-matrix $A=\left\{a_{i, j}\right\}$ from Theorem 3.9 is called the matrix of multiplicities of the weak $\mathcal{M}$-structure.

Definition 3.8. The $r \times s$-matrix $A$ is called decomposable if there exist partitions $\{1, \ldots, r\}=I \cup I^{\prime}$ and $\{1, \ldots, s\}=J \cup J^{\prime}$ such that $a_{i, j}=0$ for $(i, j) \in I \times J^{\prime}$ or for $(i, j) \in I^{\prime} \times J$.

Lemma 3.7. The matrix of multiplicities $A$ is indecomposable.

Consider (3.34) as a system of linear equations for the vector $\left(m_{1}, \ldots, m_{r}, n_{1}, \ldots, n_{s}\right)$. The matrix of the system has the form $Q=$ $\left(\begin{array}{cc}2 & -A \\ -A^{t} & 2\end{array}\right)$. According to the result by E. Vinberg [85], if the kernel of an indecomposable matrix $Q$ contains an integer positive vector, them $Q$ is the Cartan matrix of an affine Dynkin diagram. Moreover, it follows from the structure of $Q$ that this is a simple laced affine Dynkin diagram with a partition of the set of vertices into two subsets (white and black vertices on the pictures below) such that vertices of the same subset are not connected. 
Theorem 3.10. Let $A$ be an $r \times s$-matrix of multiplicities for an indecomposable weak $\mathcal{M}$-structure. Then, after a permutation of rows and columns and up to transposition, the matrix $A$ is equal to one from the following list:

1. $A=(2)$. Here $r=s=1, n_{1}=m_{1}=m$. The corresponding Dynkin diagram is of the type $\tilde{A}_{1}$.

2. $a_{i, i}=a_{i, i+1}=1$ and $a_{i, j}=0$ for other pairs $i, j$. Here $r=s=k \geq 2$, the indices are taken modulo $k$, and $n_{i}=m_{i}=m$. The corresponding Dynkin diagram is $\tilde{A}_{2 k-1}$.

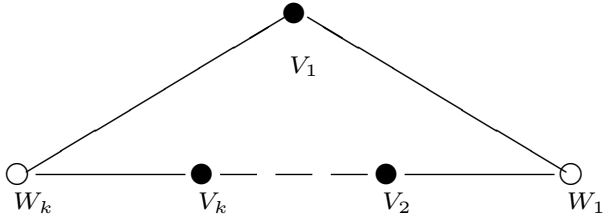

$\tilde{A}_{2 k-1}$

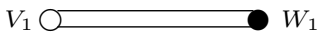

$\tilde{A}_{1}$

3. $A=\left(\begin{array}{llll}1 & 1 & 0 & 0 \\ 1 & 0 & 1 & 0 \\ 1 & 0 & 0 & 1\end{array}\right)$. Here $r=3, s=4$ and $n_{1}=3 m, n_{2}=n_{3}=$ $n_{4}=m, m_{1}=m_{2}=m_{3}=2 m$. The Dynkin diagram is $\tilde{E}_{6}$ :

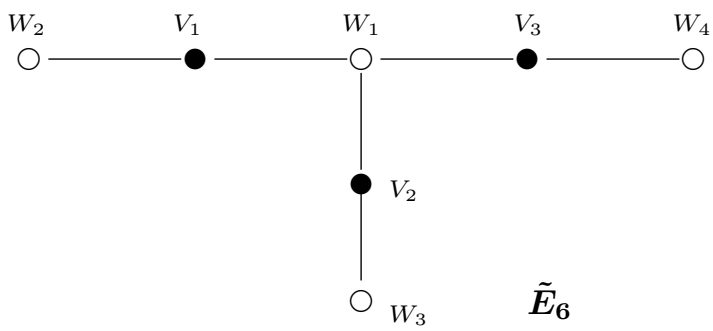

4. $A=\left(\begin{array}{lllll}1 & 1 & 0 & 0 & 0 \\ 0 & 1 & 1 & 1 & 0 \\ 0 & 0 & 0 & 1 & 1\end{array}\right)$. Here $r=3, s=5$ and $n_{1}=$ $m, \quad n_{2}=3 m, \quad n_{3}=2 m, \quad n_{4}=3 m, \quad n_{5}=m, \quad m_{1}=2 m, \quad m_{2}=$ $4 m, \quad m_{3}=2 m$. The Dynkin diagram is $\tilde{E}_{7}$ : 


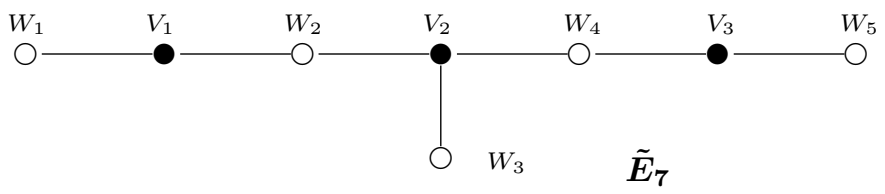

5. $A=\left(\begin{array}{lllll}1 & 0 & 0 & 0 & 0 \\ 1 & 1 & 1 & 0 & 0 \\ 0 & 0 & 1 & 1 & 0 \\ 0 & 0 & 0 & 1 & 1\end{array}\right)$. Here $r=4, s=5$ and $n_{1}=4 m, \quad n_{2}=3 m, \quad n_{3}=5 m, \quad n_{4}=3 m, \quad n_{5}=m, \quad m_{1}=$ $2 m, \quad m_{2}=6 m, \quad m_{3}=4 m, \quad m_{4}=2 m$. The Dynkin diagram is $\tilde{E}_{8}$ :

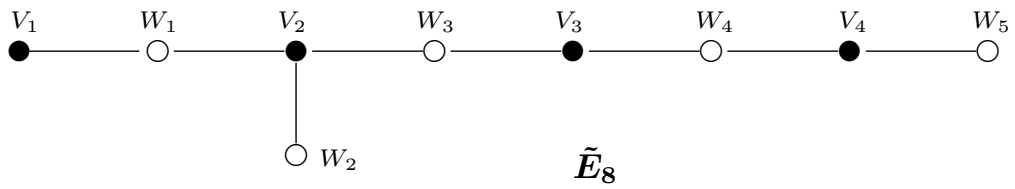

6. $A=(1,1,1,1)$. Here $r=1, s=4$ and $n_{1}=n_{2}=n_{3}=n_{4}=$ $m, m_{1}=2 m$. The corresponding Dynkin diagram is $\tilde{D}_{4}$.

7. $a_{1,1}=a_{1,2}=a_{1,3}=1, \quad a_{2,3}=a_{2,4}=a_{3,4}=a_{3,5}=\cdots=a_{k-2, k-1}=$ $a_{k-2, k}=1, \quad a_{k-1, k}=a_{k-1, k+1}=a_{k-1, k+2}=1$, and $a_{i, j}=0$ for other $(i, j)$.

Here we have $r=k-1, s=k+2$ and $n_{1}=n_{2}=n_{k+1}=n_{k+2}=$ $m, n_{3}=\cdots=n_{k}=2 m, m_{1}=\cdots=m_{l}=2 m$. The corresponding Dynkin diagram is $\tilde{D}_{2 k}$, where $k \geq 3$.

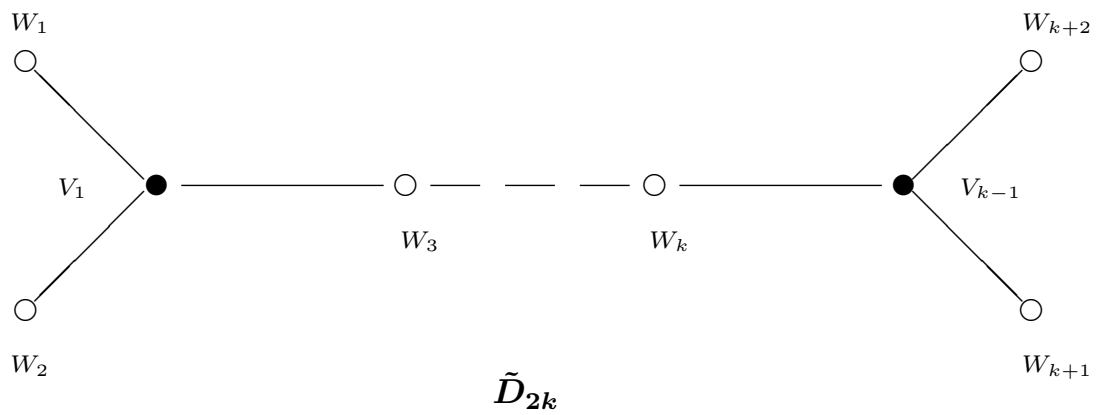


8. $a_{1,1}=a_{1,2}=a_{1,3}=1, \quad a_{2,3}=a_{2,4}=a_{3,4}=a_{3,5}=\cdots=a_{k-2, k-1}=$ $a_{k-2, k}=1, \quad a_{k-1, k}=a_{k, k}=1$, and $a_{i, j}=0$ for other $(i, j)$.

Here we have $r=s=k \geq 3, n_{1}=n_{2}=m, n_{3}=\cdots=n_{k}=2 m, m_{1}=$ $\cdots=m_{k-2}=2 m, m_{k-1}=m_{k}=m$. The corresponding Dynkin diagram is $\tilde{D}_{2 k-1}$ :

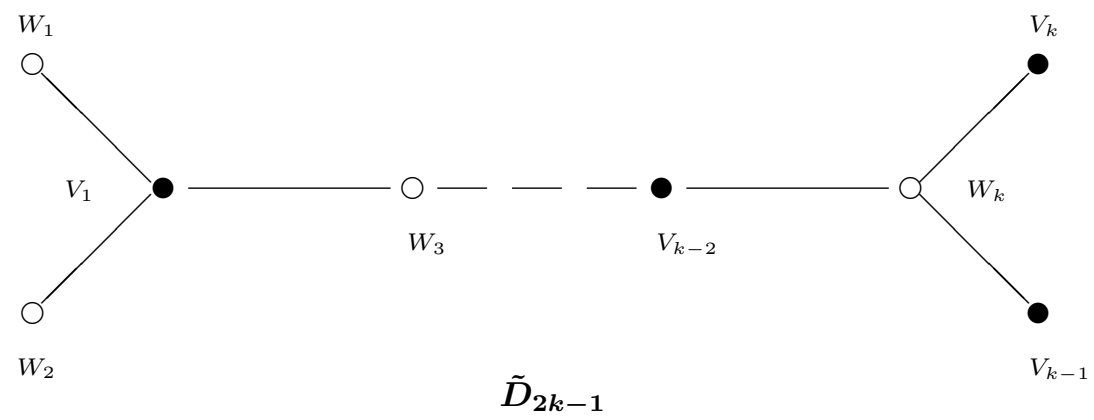

Note that if $k=3$, then $a_{1,1}=a_{1,2}=a_{1,3}=1, \quad a_{2,3}=a_{3,3}=1$.

\section{Resume}

Suppose that $\mathcal{L}$ is an indecomposable $\mathcal{M}$-structure with semi-simple algebras (3.32); then there exists an affine Dynkin diagram of the type $A, D$, or $E$ such that:

1. There is a one-to-one correspondence between the set of vertices and the set of vector spaces $\left\{V_{1}, \ldots, V_{r}, W_{1}, \ldots, W_{s}\right\}$.

2. For any $i, j$ the spaces $V_{i}, V_{j}$ are not connected by edges as well as $W_{i}, W_{j}$.

3. The vector

$$
\left(\operatorname{dim} V_{1}, \ldots, \operatorname{dim} V_{r}, \operatorname{dim} W_{1}, \ldots, \operatorname{dim} W_{s}\right)
$$

is equal to $m J$, where $J$ is the minimal imaginary positive root of the Dynkin diagram.

Remark 3.12. It can be proved that for indecomposable $\mathcal{M}$-structures $m=1$.

Given an affine Dynkin diagram of the $A, D$, or $E$-type, to define the corresponding $\mathcal{M}$-structure it remains to construct an embedding $\mathcal{A} \rightarrow \mathcal{L}$, $\mathcal{B} \rightarrow \mathcal{L}$ and a scalar product $(\cdot, \cdot)$ on the vector space $\mathcal{L}$.

If we fix an element $1 \in \mathcal{L}$, then we can define the embedding $\mathcal{A} \rightarrow \mathcal{L}$, $\mathcal{B} \rightarrow \mathcal{L}$ by the formula $a \rightarrow 1 a, b \rightarrow b 1$ for $a \in \mathcal{A}, b \in \mathcal{B}$. We may assume that 1 is a generic element of $\mathcal{L}$. 
Thus to study $\mathcal{M}$-structures corresponding to a Dynkin diagram, one should take a generic element in $\mathcal{L}=\oplus_{1 \leq i \leq r, 1 \leq j \leq s}\left(V_{i}^{\star} \otimes W_{j}\right)^{a_{i, j}}$, find its simplest canonical form by choosing basis in the vector spaces $V_{1}, \ldots, V_{r}, W_{1}, \ldots, W_{s}$, calculate the embedding $\mathcal{A} \rightarrow \mathcal{L}, \mathcal{B} \rightarrow \mathcal{L}$ and the scalar product $(\cdot, \cdot)$ on the vector space $\mathcal{L}$.

The classification of generic elements $1 \in \mathcal{L}$ up to choice of basis in the vector spaces $V_{1}, \ldots, V_{r}, W_{1}, \ldots, W_{s}$ is equivalent to the classification of irreducible representations of the quivers corresponding to our affine Dynkin diagrams and we can apply known results about these representations.

\subsection{Non-abelian Hamiltonian formalism and trace Poisson brackets}

A Poisson structure on a commutative algebra $A$ is a Lie algebra structure on $A$ given by a Lie bracket

$$
\{\cdot, \cdot\}: A \times A \mapsto A,
$$

which satisfies the Leibniz rule

$$
\{a, b c\}=\{a, b\} c+b\{a, c\}, \quad a, b, c \in A,
$$

with the right (and, hence, also with the left) argument.

It is well-known that a naive translation of this definition to the case of a non-commutative associative algebra $A$ is not very interesting because of lack of examples different from the usual commutator (for prime rings it was shown in [19]).

\subsubsection{Non-abelian Poisson brackets on free associative algebras}

Here we consider a version of the Hamiltonian formalism for free associative algebra proposed in [51].

Let $\mathcal{A}$ be free associative algebra $\mathbb{C}\left[x_{1}, \ldots, x_{N}\right]$ with the product $\circ$. For any $a \in \mathcal{A}$ we denote by $L_{a}$ (resp. $R_{a}$ ) the operators of left (resp. right) multiplication by $a$ :

$$
L_{a}(X)=a X, \quad R_{a}(X)=X a, \quad X \in \mathcal{A} .
$$

The associativity of $\mathcal{A}$ is equivalent to the identity $\left[L_{a}, R_{b}\right]=0$ for any $a$ and $b$. Moreover,

$$
L_{a b}=L_{a} L_{b}, \quad R_{a b}=R_{b} R_{a}, \quad L_{a+b}=L_{a}+L_{b}, \quad R_{a+b}=R_{a}+R_{b}
$$


Definition 3.9. We denote by $\mathcal{O}$ the associative algebra generated by all operators of left and right multiplication by elements $x_{i}$. This algebra is called the algebra of local operators.

To introduce the concept of first integrals, we need an analog of trace, which is not yet defined in the algebra $\mathcal{A}$. As a matter of fact, in our calculations we use only two properties of the trace, namely linearity and the possibility to perform cyclic permutations in monomials. Let us define an equivalence relation for elements of $\mathcal{A}$ in a standard way.

Definition 3.10. Two elements $f_{1}$ and $f_{2}$ of $\mathcal{A}$ are said to be equivalent, which we denote $f_{1} \sim f_{2}$, iff $f_{1}$ can be obtained from $f_{2}$ by cyclic permutations of generators in its monomials. We denote by $\operatorname{tr} f$ the equivalence class of the element $f$.

We are going to define a class of Poisson brackets on the functionals $\operatorname{tr} f$ (see Definition 3.10). It is easy to see that the vector space of such functionals can be identified with the quotient vector space $\mathcal{T}=\mathcal{A} /[\mathcal{A}, \mathcal{A}]$ and the Poisson brackets have to be defined on $\mathcal{T}[9]$.

Let $a(\mathbf{x}) \in \mathcal{A}$, where $\mathbf{x}=\left(x_{1}, \ldots, x_{N}\right)$ and $\delta \mathbf{x}=\left(\delta x_{1}, \ldots, \delta x_{N}\right)$, $\delta x_{i} \in \mathcal{A}$. Then $\operatorname{grad}_{\mathbf{x}}(a) \in \mathcal{A}^{N}$ is a vector

$$
\operatorname{grad}_{\mathbf{x}}(a)=\left(\operatorname{grad}_{x_{1}}(a), \ldots, \operatorname{grad}_{x_{N}}(a)\right)
$$

uniquely defined by the formula

$$
\left.\frac{d}{d \epsilon} a(\mathbf{x}+\epsilon \delta \mathbf{x})\right|_{\epsilon=0} \sim\left\langle\delta \mathbf{x}, \operatorname{grad}_{\mathbf{x}}(a(\mathbf{x}))\right\rangle,
$$

where $\left\langle\left(p_{1}, \ldots, p_{N}\right),\left(q_{1}, \ldots, q_{N}\right)\right\rangle=p_{1} \circ q_{1}+\cdots+p_{N} \circ q_{N}$.

Lemma 3.8. If $f \sim g$, then $\operatorname{grad}_{\mathbf{x}}(f)=\operatorname{grad}_{\mathbf{x}}(g)$.

It follows from the lemma that the map $\operatorname{grad}_{\mathbf{x}}: \mathcal{T} \rightarrow \mathcal{A}^{N}$ is well-defined. The Poisson brackets on $\mathcal{T}$ are defined by the formula

$$
\{f, g\}=\left\langle\operatorname{grad}_{\mathbf{x}} f, \Theta\left(\operatorname{grad}_{\mathbf{x}} g\right)\right\rangle, \quad f, g \in \mathcal{T},
$$

where

$$
\{f, g\}+\{g, f\} \sim 0, \quad\{f,\{g, h\}\}+\{g,\{h, f\}\}+\{h,\{f, g\}\} \sim 0
$$

for any elements $f, g, h \in \mathcal{T}$. Here a skew-symmetric Hamiltonian operator $\Theta$ is supposed to be an element of $\mathcal{O} \otimes \mathfrak{g l}_{N}$.

Remark 3.13. Actually, the right hand side of (3.35) is a well-defined element of $\mathcal{A}$ and we take its equivalence class for the left hand side. The left hand sides of (3.36) are regarded as elements of $\mathcal{A}$. 
It is easy to show that $(3.36)$ is equivalent to

$$
\Theta^{\star}=-\Theta
$$

i.e. $\Theta$ is a skew-symmetric operator with respect to the involution defined by

$$
L_{a}^{\star}=R_{a}, \quad R_{a}^{\star}=L_{a} .
$$

Definition 3.11. Brackets (3.35) are called non-abelian Poisson brackets.

Any Hamiltonian system of equations on $\mathcal{A}$ has the form

$$
\frac{d \mathbf{x}}{d t}=\Theta\left(\operatorname{grad}_{\mathbf{x}} H\right)
$$

where $H(\mathbf{x}) \in \mathcal{A} /[\mathcal{A}, \mathcal{A}]$ is a Hamiltonian and $\Theta$ is a Hamiltonian operator. The ODE system (3.37) has the form

$$
\frac{d x_{\alpha}}{d t}=F_{\alpha}(\mathbf{x}), \quad \mathbf{x}=\left(x_{1}, \ldots, x_{N}\right)
$$

where $F_{\alpha}$ are (non-commutative) polynomials with scalar constant coefficients. Formula (3.38) does not mean that the generators $x_{i}$ of the algebra $\mathcal{A}$ evolve in $t$. This formula defines a derivation $D_{F}$ of $\mathcal{A}$ such that $D_{F}\left(x_{i}\right)=F_{i}$. However, if we replace the generators $x_{i}$ by $m \times m$ matrices $\mathbf{x}_{i}$, then (3.38) becomes a usual system of ODEs for the entries of the matrices $\mathbf{x}_{i}$.

\section{Non-abelian Hamiltonian operators}

Consider linear Hamiltonian operators. It means that the entries of the Hamiltonian operator $\Theta$ are given by

$$
\Theta_{i j}=b_{i j}^{k} R_{x_{k}}+\bar{b}_{i j}^{k} L_{x_{k}} .
$$

The skew-symmetry of $\Theta$ implies

$$
\bar{b}_{i j}^{k}=-b_{j i}^{k} .
$$

Proposition 3.4. An operator $\Theta$ given by (3.39), (3.40) is Hamiltonian iff $b_{i j}^{k}$ are the structural constant of an associative algebra.

Corollary 3.1. Any pair of compatible associative algebras (see Section 3.2.2) generates a pair of compatible linear non-abelian Poisson brackets.

Example 3.14. Let $N=2$. Consider the following compatible associative products:

$$
x_{1} \star x_{1}=x_{1}, \quad x_{1} \star x_{2}=x_{2} \star x_{1}=x_{2} \star x_{2}=0
$$


and

$$
x_{1} \circ x_{1}=x_{2}, \quad x_{1} \circ x_{2}=x_{2} \circ x_{1}=x_{2} \circ x_{2}=0 .
$$

The corresponding Poisson brackets $\{\cdot, \cdot\}_{i}$ have the Hamiltonian operators

$$
\Theta_{i}=\left(\begin{array}{cc}
R_{x_{i}}-L_{x_{i}} & 0 \\
0 & 0
\end{array}\right), \quad i=1,2
$$

The pencil $\{\cdot, \cdot\}_{1}+\lambda\{\cdot, \cdot\}_{2}$ has a Casimir function $C=\operatorname{tr}\left(x_{1}+\lambda x_{2}\right)^{3}$, which produces the Hamiltonians $H_{1}=-\operatorname{tr}\left(x_{1}^{2} x_{2}\right)$ and $H_{2}=\frac{1}{3} \operatorname{tr}\left(x_{1}^{3}\right)$ commuting with respect to both brackets (see Theorem 1.1). The formula

$$
\frac{d \mathbf{x}}{d t}=\Theta_{1}\left(\operatorname{grad}_{\mathbf{x}} H_{1}\right)=\Theta_{2}\left(\operatorname{grad}_{\mathbf{x}} H_{2}\right)
$$

gives us a bi-Hamiltonian representation for the system

$$
\frac{d x_{1}}{d t}=x_{1}^{2} x_{2}-x_{2} x_{1}^{2}, \quad \frac{d x_{2}}{d t}=0
$$

already mentioned in Examples 1.1, 3.10 and in Application 3.1.

For quadratic Poisson brackets the general form of the Hamiltonian operator is given by

$$
\Theta_{i, j}=a_{i j}^{p q} L_{x_{p}} L_{x_{q}}-a_{j i}^{q p} R_{x_{p}} R_{x_{q}}+r_{i j}^{p q} L_{x_{p}} R_{x_{q}}
$$

where $a_{i j}^{p q}$ and $r_{i j}^{p q}$ are some (complex) constants, $r_{i j}^{p q}=-r_{j i}^{q p}, p, q, i, j=$ $1, \ldots, N$, and the summation over repeated indices is assumed.

Proposition 3.5. Formula (3.41) define a Poisson bracket iff the following relations hold:

$$
\begin{gathered}
r_{\alpha \beta}^{\sigma \epsilon}=-r_{\beta \alpha}^{\epsilon \sigma}, \\
r_{\alpha \beta}^{\lambda \sigma} r_{\sigma \tau}^{\mu \nu}+r_{\beta \tau}^{\mu \sigma} r_{\sigma \alpha}^{\nu \lambda}+r_{\tau \alpha}^{\nu \sigma} r_{\sigma \beta}^{\lambda \mu}=0, \\
a_{\alpha \beta}^{\sigma \lambda} a_{\tau \sigma}^{\mu \nu}=a_{\tau \alpha}^{\mu \sigma} a_{\sigma \beta}^{\nu \lambda}, \\
a_{\alpha \beta}^{\sigma \lambda} a_{\sigma \tau}^{\mu \nu}=a_{\alpha \beta}^{\mu \sigma} r_{\tau \sigma}^{\lambda \nu}+a_{\alpha \sigma}^{\mu \nu} r_{\beta \tau}^{\sigma \lambda} .
\end{gathered}
$$

and

$$
a_{\alpha \beta}^{\lambda \sigma} a_{\tau \sigma}^{\mu \nu}=a_{\alpha \beta}^{\sigma \nu} r_{\sigma \tau}^{\lambda \mu}+a_{\sigma \beta}^{\mu \nu} r_{\tau \alpha}^{\sigma \lambda}
$$

Remark 3.14. Conditions (3.42) and (3.43) mean that the tensor $\mathbf{r}$ satisfies the associative Yang-Baxter (or Rota-Baxter) equation [64, 3]. 


\subsubsection{Trace Poisson brackets}

The non-abelian brackets are defined between traces only. But if $x_{1}, \ldots, x_{N}$ are $m \times m$-matrices, we can extend these brackets to the matrix entries.

We consider $\mathrm{Nm}^{2}$-dimensional Poisson brackets defined on functions in entries $x_{i, \alpha}^{j}$ of $m \times m$-matrices $x_{\alpha}$. Here and in the sequel, we use Latin indices ranging from 1 to $m$ for the matrix entries and Greek indices ranging from 1 to $N$ to label the matrices.

Definition 3.12. Such a bracket is called trace Poisson bracket iff

- the bracket is $\mathrm{GL}(m)$-invariant;

- for any two matrix polynomials $P_{i}\left(x_{1}, \ldots, x_{N}\right), i=1,2$ with coefficients in $\mathbb{C}$ the bracket between its traces is the trace of some matrix polynomial $P_{3}$.

Theorem 3.11. Any constant trace Poisson bracket has the form

$$
\left\{x_{i, \alpha}^{j}, x_{i^{\prime}, \beta}^{j^{\prime}}\right\}=\delta_{i^{\prime}}^{j} \delta_{i}^{j^{\prime}} c_{\alpha \beta}
$$

Any linear trace Poisson bracket has the form

$$
\left\{x_{i, \alpha}^{j}, x_{i^{\prime}, \beta}^{j^{\prime}}\right\}=b_{\alpha, \beta}^{\gamma} x_{i, \gamma}^{j^{\prime}} \delta_{i^{\prime}}^{j}-b_{\beta, \alpha}^{\gamma} x_{i^{\prime}, \gamma}^{j} \delta_{i}^{j^{\prime}}
$$

Any quadratic trace Poisson bracket is given by

$$
\left\{x_{i, \alpha}^{j}, x_{i^{\prime}, \beta}^{j^{\prime}}\right\}=r_{\alpha \beta}^{\gamma \epsilon} x_{i, \gamma}^{j^{\prime}} x_{i^{\prime}, \epsilon}^{j}+a_{\alpha \beta}^{\gamma \epsilon} x_{i, \gamma}^{k} x_{k, \epsilon}^{j^{\prime}} \delta_{i^{\prime}}^{j}-a_{\beta \alpha}^{\gamma \epsilon} x_{i^{\prime}, \gamma}^{k} x_{k, \epsilon}^{j} \delta_{i}^{j^{\prime}} .
$$

Moreover

1) Formula (3.47) defines a Poisson bracket iff

$$
c_{\alpha \beta}=-c_{\beta \alpha}
$$

2) Formula (3.48) defines a Poisson bracket iff

$$
b_{\alpha \beta}^{\mu} b_{\mu \gamma}^{\sigma}=b_{\alpha \mu}^{\sigma} b_{\beta \gamma}^{\mu}
$$

3) Formula (3.49) defines a Poisson brackets iff conditions (3.42)-(3.46) hold.

Remark 3.15. Formula (3.50) means that $b_{\alpha \beta}^{\sigma}$ are the structure constants of an associative algebra $\mathcal{A}$. A straightforward verification shows that (3.48) is nothing but the Lie-Kirillov-Kostant bracket defined by the Lie algebra corresponding to the associative algebra $\mathrm{Mat}_{m} \otimes \mathcal{A}$.

Lemma 3.9. For any Hamiltonian of the form $H=\operatorname{tr} P$, where $P$ is a matrix polynomial, the equations of motion can be written in the matrix form (3.38). 
Under a linear transformation of the matrices $x_{i} \rightarrow g_{i}^{j} x_{j}$ the constants in (3.49) are transformed in a standard way:

$$
r_{i j}^{k l} \rightarrow g_{i}^{\alpha} g_{j}^{\beta} h_{\gamma}^{k} h_{\epsilon}^{l} r_{\alpha, \beta}^{\gamma, \epsilon}, \quad a_{i j}^{k l} \rightarrow g_{i}^{\alpha} g_{j}^{\beta} h_{\gamma}^{k} h_{\epsilon}^{l} a_{\alpha, \beta}^{\gamma, \epsilon} .
$$

Here $g_{i}^{j} h_{j}^{k}=\delta_{i}^{k}$.

Definition 3.13. Two brackets of the form (3.49) related by (3.51) are called equivalent.

A relation between non-abelian and trace Poisson brackets can be established via the formula

$$
x_{i, \alpha}^{j}=\operatorname{tr}\left(e_{j}^{i} x_{\alpha}\right), \quad x_{i^{\prime}, \beta}^{j^{\prime}}=\operatorname{tr}\left(e_{j^{\prime}}^{i^{\prime}} x_{\beta}\right),
$$

where $e_{j}^{i}$ stand for the matrix unities. For instance, consider the Hamiltonian operator (3.41). Applying formula (3.35) and using the definition of the gradient, we arrive at (3.49).

Identities (3.42)-(3.46) can be rewritten in a tensor form. Let $\mathbf{V}$ be a linear space with a basis $\mathbf{e}_{i}, i=1, \ldots, N$. Define linear operators $R$ and $A$ on the space $\mathbf{V} \otimes \mathbf{V}$ by $R \mathbf{e}_{i} \otimes \mathbf{e}_{j}=r_{i j}^{p q} \mathbf{e}_{p} \otimes \mathbf{e}_{q}, A \mathbf{e}_{i} \otimes \mathbf{e}_{j}=a_{i j}^{p q} \mathbf{e}_{p} \otimes \mathbf{e}_{q}$. Then identities (3.42) - (3.46) are equivalent to

$$
\begin{gathered}
R^{12}=-R^{21}, \\
R^{23} R^{12}+R^{31} R^{23}+R^{12} R^{31}=0, \\
A^{12} A^{31}=A^{31} A^{12}, \\
\sigma^{23} A^{13} A^{12}=A^{12} R^{23}-R^{23} A^{12}, \\
A^{32} A^{12}=R^{13} A^{12}-A^{32} R^{13} .
\end{gathered}
$$

Here all operators act on $\mathbf{V} \otimes \mathbf{V} \otimes \mathbf{V}$, by $\sigma^{i j}$ we mean transposition of $i$-th and $j$-th components of the tensor product and $A^{i j}, R^{i j}$ mean operators $A, R$ acting on the tensor product of the $i$-th and $j$-th components.

The equivalence transformation (3.51) corresponds to $A \rightarrow G A G^{-1}, R \rightarrow$ $G R G^{-1}$, where $G=g \otimes g$ and $g \in \operatorname{End}(\mathbf{V})$.

Definition 3.14. (cf.(1.20)) A vector $\Lambda=\left(\lambda_{1}, \ldots, \lambda_{N}\right)$ is said to be admissible for (3.49) if for any $i, j$

$$
\left(a_{i j}^{p q}-a_{j i}^{q p}+r_{i j}^{p q}\right) \lambda_{p} \lambda_{q}=0 .
$$

Lemma 3.10. For any admissible vector the argument shift $x_{i} \rightarrow x_{i}+\lambda_{i}$ Id in (3.49) yields a linear Poisson bracket (3.48), where

$$
b_{i j}^{p}=\left(a_{i j}^{q p}+a_{i j}^{p q}+r_{i j}^{p q}\right) \lambda_{q}
$$

compatible with (3.49). 


\section{Case $\mathbf{a}=0$ and anti-Frobenius algebras}

There is a subclass of brackets (3.49) that corresponds to the case when the tensor $\mathbf{a}$ is equal to 0 . Relations (3.42), (3.43) mean that the tensor $\mathbf{r}$ is a constant solution of the associative Yang-Baxter equation ([3], [67]). Such solutions can be constructed in the following algebraic way.

Definition 3.15. An anti-Frobenius algebra is an associative algebra $\mathcal{A}$ (not necessarily with unity) with non-degenerate anti-symmetric bilinear form $($, ) satisfying the following relation

$$
(x, y \circ z)+(y, z \circ x)+(z, x \circ y)=0
$$

for all $x, y, z \in \mathcal{A}$. In other words the form ( , ) defines an 1-cocycle on $\mathcal{A}$.

Theorem 3.12. There exists a one-to-one correspondence between solutions of (3.42), (3.43) up to equivalence and exact representations of anti-Frobenius algebras up to isomorphism.

Proof. The tensor $\mathbf{r}$ can be written as $r_{k l}^{i j}=\sum_{\alpha, \beta=1}^{p} g^{\alpha \beta} y_{k, \alpha}^{i} y_{l, \beta}^{j}$, where $g^{\alpha \beta}=-g^{\beta \alpha}$, the matrix $G=\left(g^{\alpha \beta}\right)$ is non-degenerate and $p$ is the smallest possible. Substituting this expression into (3.42), (3.43), we obtain that there exists a tensor $\phi_{\alpha \beta}^{\gamma}$ such that $y_{k, \alpha}^{i} y_{j, \beta}^{k}=\phi_{\alpha \beta}^{\gamma} y_{j, \gamma}^{i}$. Let $\mathcal{A}$ be an associative algebra with basis $y_{1}, \ldots, y_{p}$ and product $y_{\alpha} \circ y_{\beta}=\phi_{\alpha \beta}^{\gamma} y_{\gamma}$. Define the anti-symmetric bilinear form by $\left(y_{\alpha}, y_{\beta}\right)=g_{\alpha \beta}$, where $\left\{g_{\alpha \beta}\right\}=$ $G^{-1}$. Then (3.42), (3.43) are equivalent to the anti-Frobenius property (3.52).

Example 3.15. (cf. [17]). Let $\mathcal{A}$ be the associative algebra of $N \times N$ matrices with zero $N$-th row. For a generic element $l$ of $\mathcal{A}^{*}$; the bilinear form $(x, y)=l([x, y])$ is a non-degenerate anti-symmetric form, which satisfies (3.52). It can be written as $(x, y)=\operatorname{tr}\left([x, y] k^{T}\right)$, where $k \in \mathcal{A}$. Let us choose $\quad k_{i j}=0, i \neq j, \quad k_{i i}=\mu_{i}$, where $i, j=1, \ldots, N-1$, and $k_{i N}=1, i=1, \ldots, N-1$. The corresponding bracket (3.49) is given by the following tensor $\mathbf{r}$ :

$$
r_{N i}^{i i}=-r_{i N}^{i i}=1, \quad r_{i j}^{i j}=r_{i j}^{j i}=r_{j i}^{i i}=-r_{i j}^{i i}=\frac{1}{\mu_{i}-\mu_{j}},
$$

where $i \neq j, \quad i, j=1, \ldots, N-1$. The remaining elements of the tensor $\mathbf{r}$ and all elements of the tensor a are supposed to be zero. It can be verified that (3.53) is equivalent to the bracket given by

$$
r_{\alpha \beta}^{\alpha \beta}=r_{\alpha \beta}^{\beta \alpha}=r_{\beta \alpha}^{\alpha \alpha}=-r_{\alpha \beta}^{\alpha \alpha}=\frac{1}{\lambda_{\alpha}-\lambda_{\beta}}, \quad \alpha \neq \beta, \quad \alpha, \beta=1, \ldots, N .
$$

Here $\lambda_{1}, \ldots, \lambda_{N}$ are arbitrary pairwise distinct parameters. Formula (3.49) with zero tensor a defines the corresponding trace Poisson bracket for 
entries of matrices $x_{1}, \ldots, x_{N}$ of arbitrary size $m$. For $m=1$ we have the scalar Poisson bracket

$$
\left\{x_{\alpha}, x_{\beta}\right\}=\frac{\left(x_{\alpha}-x_{\beta}\right)^{2}}{\lambda_{\beta}-\lambda_{\alpha}}, \quad \alpha \neq \beta, \quad \alpha, \beta=1, \ldots, N .
$$

If $N$ is even, then the trace Poisson structure (3.54) is non-degenerate, i.e. the rank of the Poisson tensor $\Pi$ is equal to $\mathrm{Nm}^{2}$. In the odd case $\operatorname{rank} \Pi=(N-1) m^{2}$.

Remark 3.16. Bracket (3.54) can be directly obtained from the antiFrobenius algebra

$$
\mathcal{A}_{N, 1}=\left\{A \in \operatorname{Mat}_{N} \mid \sum_{i} a_{i j}=0 \quad \forall j=1, \ldots, N\right\}
$$

equipped with the bilinear form

$$
(x, y)=\operatorname{tr}\left([x, y] \cdot \operatorname{diag}\left(\lambda_{1}, \ldots, \lambda_{N}\right)\right) .
$$

In [90] the algebra $\mathcal{A}_{N, 1}$ was generalized. Let $M$ be a proper divisor of $N$. We consider $N(N-M)$-dimensional algebra

$$
\mathcal{A}_{N, M}=\left\{A \in \operatorname{Mat}_{N} \mid \sum_{i \equiv r} a_{(\bmod M)} a_{i j}=0 \quad \forall r=1, \ldots, M, \forall j=1, \ldots, N\right\}
$$

equipped with the bilinear form (3.55). Suppose that $\lambda_{i}$ are pairwise distinct. One can check that in this case the form $(x, y)$ is non-degenerate [17]. The components of the tensor $\mathbf{r}$ corresponding to the algebra $\mathcal{A}_{N, M}$ are given by

$$
\begin{gathered}
r_{\gamma \delta}^{\alpha \beta}=0, \quad \text { if } \alpha \neq \equiv \delta \quad \text { or } \quad \beta \neq \gamma, \\
r_{\varepsilon \alpha}^{\alpha \alpha}=-r_{\alpha \varepsilon}^{\alpha \alpha}=\frac{1}{\lambda_{\alpha}-\lambda_{\varepsilon}}, \quad \text { when } \alpha \neq \varepsilon, \\
r_{\gamma \delta}^{\alpha \alpha}=0, \quad \text { if } \gamma \neq \alpha \quad \text { or } \quad \delta \neq \alpha, \\
r_{\beta \alpha}^{\alpha \beta}=\frac{1}{\lambda_{\alpha}-\lambda_{\beta}}\left(\frac{\prod_{\beta^{\prime} \equiv \beta, \beta^{\prime} \neq \beta}\left(\lambda_{\alpha}-\lambda_{\beta^{\prime}}\right) \prod_{\alpha^{\prime} \equiv \alpha, \alpha^{\prime} \neq \alpha}\left(\lambda_{\beta}-\lambda_{\alpha^{\prime}}\right)}{\prod_{\alpha^{\prime} \neq \alpha}\left(\lambda_{\alpha}-\lambda_{\alpha^{\prime}}\right) \prod_{\beta^{\prime} \equiv \beta, \beta^{\prime} \neq \beta}\left(\lambda_{\beta}-\lambda_{\beta^{\prime}}\right)}-1\right), \quad \text { if } \alpha \neq \beta, \\
r_{\gamma \delta}^{\alpha \beta}=\frac{1}{\lambda_{\alpha}-\lambda_{\beta}} \cdot \frac{\prod_{\gamma^{\prime} \equiv \gamma, \gamma^{\prime} \neq \gamma}\left(\lambda_{\alpha}-\lambda_{\gamma^{\prime}}\right) \prod_{\delta^{\prime} \equiv \delta, \delta^{\prime} \neq \delta}\left(\lambda_{\beta}-\lambda_{\delta^{\prime}}\right)}{\prod_{\alpha^{\prime} \equiv \alpha, \alpha^{\prime} \neq \alpha}\left(\lambda_{\alpha}-\lambda_{\alpha^{\prime}}\right) \prod_{\beta^{\prime} \equiv \beta, \beta^{\prime} \neq \beta}\left(\lambda_{\beta}-\lambda_{\beta^{\prime}}\right)} \quad \text { otherwise. }
\end{gathered}
$$

Here $x \equiv y$ means that $x=y(\bmod M)$. 
With these formulas one can construct corresponding quadratic Poisson brackets. For example, in the case $N=2 M$ and $m=1$ the corresponding scalar Poisson bracket has the form

$$
\left\{x_{\alpha}, x_{\beta}\right\}=\frac{\left(x_{\alpha}-x_{\alpha^{\prime}}\right)\left(x_{\beta}-x_{\beta^{\prime}}\right)\left(\lambda_{\alpha^{\prime}}-\lambda_{\beta^{\prime}}\right)}{\left(\lambda_{\alpha}-\lambda_{\alpha^{\prime}}\right)\left(\lambda_{\beta}-\lambda_{\beta^{\prime}}\right)},
$$

where for any $\gamma$ the positive integer $\gamma^{\prime}$ is uniquely defined by the condition $\left|\gamma^{\prime}-\gamma\right|=M$.

Open problem 3.6. Describe all anti-Frobenius algebras $\mathcal{A}$ of the form

$$
\mathcal{A}=\mathcal{S} \oplus \mathcal{M}
$$

where $\mathcal{S}$ is a semi-simple associative algebra and $\mathcal{M}$ is a $\mathcal{S}$-module such that $\mathcal{M}^{2}=\{0\}$.

\section{Classification of trace quadratic brackets for $N=2$}

Consider the case $N=2$.

Theorem 3.13. Any Poisson bracket (3.49) up to transformations (3.51) and to the proportionality is one of the following brackets. Here we present non-zero components of the tensors $\mathbf{a}$ and $\mathbf{r}$ only.

- $r_{12}^{22}=1, \quad r_{21}^{22}=-1$;

- $r_{11}^{21}=1, \quad r_{11}^{12}=-1, \quad a_{21}^{22}=a_{11}^{12}=-1$;

- $r_{11}^{21}=-1, \quad r_{11}^{12}=1, \quad a_{12}^{22}=a_{11}^{21}=1$;

- $r_{12}^{22}=1, \quad r_{21}^{22}=-1, \quad a_{11}^{12}=a_{21}^{22}=1$;

- $r_{21}^{22}=1, \quad r_{12}^{22}=-1, \quad a_{11}^{21}=a_{12}^{22}=1$;

- $a_{11}^{22}=1$;

- $r_{11}^{21}=1, \quad r_{11}^{12}=-1$.

For a classification in the case $N=3, \mathbf{a}=0$ see [73].

Open problem 3.7. Describe all trace Poisson brackets (3.49) for $N=3$.

\subsubsection{Double Poisson brackets on free associative algebra}

In the previous subsections we observed that identities (3.42)-(3.46) describe both non-abelian and trace quadratic Poisson brackets. Here we show that this is also true for the quadratic double Poisson brackets on the free associative algebra with generators $x_{1}, \ldots, x_{N}$. 
Definition 3.16. [82]. A double Poisson bracket on an associative algebra $A$ is a $\mathbb{C}$-linear map $\{\{\}\}: A \otimes A \rightarrow A \otimes A$ satisfying the following conditions:

$$
\begin{gathered}
\{\{u, v\}\}=-\left\{\{v, u\}^{\circ},\right. \\
\left\{\{u,\{\{v, w\}\}\}+\sigma\left\{\{v,\{\{w, u\}\}\}+\sigma^{2}\{\{w,\{\{u, v\}\}\}=0,\right.\right.
\end{gathered}
$$

and

$$
\{\{u, v w\}=(v \otimes 1)\{\{u, w\}+\{\{u, v\}(1 \otimes w) .
$$

Here $(u \otimes v)^{\circ} \stackrel{\text { def }}{=} v \otimes u ; \quad\left\{v_{1}, v_{2} \otimes v_{3}\right\}:=\left\{\left\{v_{1}, v_{2}\right\} \otimes v_{3} \quad\right.$ and $\sigma\left(v_{1} \otimes v_{2} \otimes v_{3}\right):=v_{3} \otimes v_{1} \otimes v_{2}$.

Denote by $\mu$ the multiplication map $\mu: A \otimes A \rightarrow A$ given by $\mu(u \otimes v)=$ $u v$. We define a $\mathbb{C}$-bilinear bracket operation on $A$ by $\{\cdot, \cdot\} \stackrel{\text { def }}{=} \mu(\{[\cdot, \cdot\})$.

Proposition 3.6. Let $\{\{\cdot, \cdot\}$ be a double Poisson bracket on $A$. Then $\{\cdot, \cdot\}$ is a trace bracket on $A /[A, A]$, which is defined as

$$
\{\bar{a}, \bar{b}\}=\overline{\mu(\{\{a, b]\}),}
$$

where $\bar{a}$ means the image of $a \in A$ under the natural projection $A \rightarrow$ $A /[A, A]$.

Let $\mathcal{A}=\mathbb{C}\left[x_{1}, \ldots, x_{N}\right]$ be the free associative algebra. If the double brackets $\left\{x_{i}, x_{j}\right\}$ between all generators $x_{i}$ are fixed, then the bracket between two arbitrary elements of $\mathcal{A}$ is uniquely determined. Constant, linear, and quadratic double brackets are defined by

$$
\begin{gathered}
\left\{\left\{x_{i}, x_{j}\right\}\right\}=c_{i j} 1 \otimes 1, \quad c_{i, j}=-c_{j, i}, \\
\left\{\left\{x_{i}, x_{j}\right\}\right\}=b_{i j}^{k} x_{k} \otimes 1-b_{j i}^{k} 1 \otimes x_{k},
\end{gathered}
$$

and

$$
\left\{\left\{x_{\alpha}, x_{\beta}\right\}=r_{\alpha \beta}^{u v} x_{u} \otimes x_{v}+a_{\alpha \beta}^{v u} x_{u} x_{v} \otimes 1-a_{\beta \alpha}^{u v} 1 \otimes x_{v} x_{u},\right.
$$

respectively.

Proposition 3.7. These formulas define double Poisson brackets iff the constants $c_{i j}, b_{i j}^{k}, r_{i j}^{p q}, a_{i j}^{p q}$ satisfy the identities of Theorem 3.11. 


\section{Bibliography}

[1] M. J. Ablowitz and H. Segur, Solitons and the Inverse Scattering Transform, 1981, SIAM, Philadelphia, 425 pp.

[2] M. Adler, On a trace functional for formal pseudo differential operators and the symplectic structure of the Korteweg de Vries type equations, Invent. Math., 1979, 50(3), 219-248,

[3] M. Aguiar, On the associative analog of Lie bialgebras, J.Alg., 2001, 244(2), 492-532.

[4] M. Audin, Spinning Tops: A Course on Integrable Systems, Cambridge University Press, 1999, 148 pp.

[5] A. A. Belavin and V. G. Drinfeld, Solutions of the classical YangBaxter equation for simple Lie algebras, Funct. Anal. Appl., 1982, 16(3), 159-180.

[6] A. V. Borisov and I. S. Mamaev, Rigid body dynamics, Izhevsk, Chaotic and Regular Dynamics, 2001, 384 pp. (in Russian)

[7] I. V. Cherednik, Functional realizations of basis representations of factoring Lie groups and algebras, Funct. Anal. Appl., 1985, 19(3), $36-52$.

[8] I. V. Cherednik, On integrability of two-dimensional assimetric chiral O(3)-field and its quantum analog, Nuclear Physics, 1981, 33, 278-282.

[9] W. Crawley-Boevey, Poisson structures on moduli spaces of representations, J. Algebra, 2011, 325, 205-215.

[10] M. F. De Groot, T. J. Hollowood and J. L. Miramontes, Generalized Drinfeld-Sokolov hierarchies, Comm. Math. Phys., 1992, 145, 57-84. 
[11] A. De Sole, V. G. Kac and D. Valeri, Classical affine W-algebras for $\mathfrak{g l}_{N}$ and associated integrable Hamiltonian hierarchies, Comm. Math. Phys, 2016, 348(1), 265-319.

[12] V. G. Drinfeld and V. V. Sokolov, Lie algebras and equations of Korteweg de Vries type, Jour. Sov. Math., 1985, 30, 1975-2036.

[13] V. G. Drinfeld and V. V. Sokolov, New evolution equations possess (L-A)-pairs, Proceedings of S.L. Sobolev seminar, 1981, 2, 5-9.

[14] B. A. Dubrovin, Geometry of 2D topological field theories. In Integrable Systems and Quantum Groups, Lecture Notes in Math., 1996, 1620, 120-348.

[15] O. V. Efimovskaya, Factorization of the loop algebra over $\mathfrak{s o ( 4 )}$ and integrable nonlinear differential equations, Fundamental and applied mathematics, 2005, 11(3), 79-94.

[16] O. V. Efimovskaya and V. V. Sokolov, Decomposition of the loop algebra over $\mathfrak{s o ( 4 )}$ and integrable models of the chiral type, Fundamental and applied mathematics, 2004, 10(1), 39-47.

[17] A. G. Elashvili, Frobenius Lie algebras, Functional Anal. Appl., 1983, 16(4), 326-328.

[18] L. D. Faddeev and L. A. Takhtajan, The Hamiltonian Methods in the Theory of Solitons, 2007, Berlin, Springer, 592 pp.

[19] D. Farkas and G. Letzter, Ring theory from symplectic geometry, J. Pure Appl. Algebra, 1998, 125(1-3), 155-190.

[20] B. L. Feigin and A. V. Odesskii, Functional realization of some elliptic Hamiltonian structures and bozonization of the corresponding quantum algebras, Integrable Structures of Exactly Solvable Two-Dimensional Models of Quantum Field Theory, (S. Pakuliak et al., eds.), NATO Sci. Ser. II Math. Phys. Chem. Klumer, Dordrecht, 2001, 35, 109-122.

[21] A. P. Fordy and J. Gibbons, Factorization of operators I : Miura transformations, J. Math. Phys., 1980, 21, 2508-2510.

[22] I. M. Gelfand and L. A. Dikii, Asymptotic behavior of the resolvent of Sturm-Liouville equations and the algebra of the Korteweg-de Vries equations, Russian Math. Surveys, 1975, 30(5), 77-113.

[23] I. M. Gelfand and L. A. Dikii, Fractional powers of operator and Hamiltonian systems, Funct. Anal. Appl., 1976, 10(4), 259-273. 
[24] I. M. Gelfand and I. Zakharevich, Lenard schemes, and the local geometry of bi-Hamiltonian Toda and Lax structures, Selecta Math. (N.S.), 2000, 6(2), 131-183.

[25] I. Z. Golubchik and V. V. Sokolov, Factorization of the loop algebras and compatible Lie brackets, Journal of Nonlinear Math. Phys., 2005, 12(1), 343-350.

[26] I. Z. Golubchik and V. V. Sokolov, Compatible Lie brackets and integrable equations of the principle chiral model type, Func. Anal. and Appl., 2002, 36(3), 172-181.

[27] I. Z. Golubchik and V. V. Sokolov, Compatible Lie brackets and Yang-Baxter equation, Theoret. and Math. Phys., 2006, 146(2), 159-169.

[28] I. Z. Golubchik and V. V. Sokolov, Factorization of the loop algebra and integrable top-like systems, Theoret. and Math. Phys., 2004, 141(1), 1329-1347.

[29] I. Z. Golubchik and V. V. Sokolov, Generalized Heizenberg equations on Z-graded Lie algebras, Theoret. and Math. Phys., 1999, 120(2), 1019-1025.

[30] I. Z. Golubchik and V. V. Sokolov, Generalized operator YangBaxter equations, integrable ODEs and nonassociative algebras, Nonlinear Math. Phys., 2000, 7(2), 1-14.

[31] I. Z. Golubchik and V. V. Sokolov, Integrable equations on $\mathbb{Z}$ graded Lie algebras, Theoret. and Math. Phys., 1997, 112(3), 1097 $-1103$.

[32] I. Z. Golubchik and V. V. Sokolov, Multicomponent generalization of the hierarchy of the Landau-Lifshitz equation, Theor. and Math. Phys, 2000, 124(1), 909-917.

[33] I. Z. Golubchik and V. V. Sokolov, On some generalizations of the factorization method, Theoret. and Math. Phys., 1997, 110(3), 267 $-276$.

[34] I. Z. Golubchik, V. V. Sokolov and S. I. Svinolupov, A new class of nonassociative algebras and a generalized factorization method, Schrödinger Inst. preprint serie, 1993, Wien, Preprint 53, 19 p.

[35] M. Gurses, A. Karasu, and V. V. Sokolov, On construction of recursion operator from Lax representation, JMPh., 1999, 40(12), $6473-6490$. 
[36] I. Kaplansky, An introduction to differential algebra, 1957, Paris, Hermann, 63 pp.

[37] D. J. Kaup, On the inverse scattering problem for the cubic eigenvalue problem of the class $\varphi_{x x x}+6 Q \phi_{x}+6 R \phi=\lambda \phi$, Stud. Appl. Math., 1980, 62, 189-216.

[38] B. Khesin, A. Levin and M. Olshanetsky, Bi-Hamiltonian structures and quadratic algebras in hydrodynamics and on noncommutative torus, Commun. Math. Phys., 2004, 250, 581-612.

[39] B. Kostant, Quantization and representation theory, in Lect. Notes, 1979, 34, 287-316.

[40] I. M. Krichever, Vector bundles and Lax equations on algebraic curves, Comm. Math. Phys., 2002, 229(2), 229-269.

[41] I. M. Krichever and D. H. Phong, Symplectic forms in the theory of solitons, 1997, arXiv 9708170 in hep-th.

[42] I. M. Krichever and O. K. Sheinman, Lax operator algebras, Funct. Anal. Appl., 2007, 41(4), 284-294.

[43] P. Lax, Integrals of nonlinear equations of evolution and solitary waves, Comm. Pure Applied Math., 1968, 21(5), 467-490.

[44] F. Magri, A simple model of the integrable Hamiltonian equation, J. Math. Phys., 1978, 19, 1156-1162.

[45] F. Magri, P. Casati, G. Falqui and M. Pedroni, Eight lectures on Integrable Systems, In: Integrability of Nonlinear Systems (Y. Kosmann-Schwarzbach et al. eds.), Lecture Notes in Physics (2nd edition), 2004, 495, 209-250.

[46] S. V. Manakov, Note on the integration of Euler's equations of the dynamics of an n-dimensional rigid body, Funct. Anal. Appl., 1976, $\mathbf{1 0}(4), 93-94$.

[47] S. V. Manakov, On the theory of two-dimensional stationary selffocusing electromagnetic waves. Sov. Phys. JETP, 1974, 38(2), $248-253$.

[48] M. G. Matushko and V. V. Sokolov, Polynomial forms for quantum elliptic Calogero-Moser Hamiltonians, Theor. and Math. Phys., 2017, 191(1), 480-490.

[49] A. V. Mikhailov and A. B. Shabat, Integrable deformations of the Heisenberg model, Phys. Lett. A, 1986, 116(4), 191-194. 
[50] A. V. Mikhailov, A. B. Shabat and V. V. Sokolov, Symmetry Approach to Classification of Integrable Equations, In What is integrability?, 1981, Ed. V.E. Zakharov, Springer Series in Nonlinear Dynamics, Springer-Verlag, 115-184.

[51] A. V. Mikhailov and V. V. Sokolov, Integrable ODEs on Associative Algebras, Comm. in Math. Phys., 2000, 211(1), 231-251.

[52] A. S. Mischenko and A. T. Fomenko, Euler equations on finitedimensional Lie groups, Mathematics of the USSR-Izvestiya, 1978, $\mathbf{1 2}(2), 371-389$.

[53] A. Newell, Solutions in Mathematics and Physics, SIAM, Philadelphia 1985, 244 pp.

[54] S. P. Novikov, S. V. Manakov, L. P. Pitaevskii, and V. E. Zakharov, Theory of Solitons: The Inverse Scattering Method, 1984, Springer-Verlag, 320 pp.

[55] A. V. Odesskii and V. V. Sokolov, Algebraic structures connected with pairs of compatible associative algebras, International Mathematics Research Notices, 2006, ID 43734, 1-35.

[56] A. V. Odesskii and V. V. Sokolov, Compatible Lie brackets related to elliptic curve, Journal of Math. Phys., 2006, 47(1), 013506, 114 .

[57] A. V. Odesskii and V. V. Sokolov, Integrable matrix equations related to pairs of compatible associative algebras, Journal Phys. A: Math. Gen., 2006, 39, 12447-12456.

[58] A. V. Odesskii and V. V. Sokolov, Pairs of compatible associative algebras, classical Yang-Baxter equation and quiver representations, Comm. in Math. Phys., 2008, 278(1), 83-99.

[59] M. A. Olshanetsky and A. M. Perelomov, Quantum integrable systems related to Lie algebras, Phys. Repts., 1983, 94, 313-393.

[60] P. J. Olver, Applications of Lie Groups to Differential Equations, (2nd edn), Graduate Texts in Mathematics, 1993, 107, SpringerVerlag, New York, 513 pp.

[61] O. Ore, Theory of non-commutative polynomials, Ann. Math., 1933, 34, 480-508.

[62] V. Ostapenko, Endomorphisms of lattices of a Lie algebra over formal power series field, C.R. Acad. Sci., 1992. Paris, 315, Serie 1, p. 669-673. 
[63] A. G. Reyman and M. A. Semenov-Tian-Shansky, Integrable systems. Group theory approach, 2003, Izhevsk, ICS Publ., 350 pp.

[64] G. C. Rota, Baxter operators, an introduction in "Gian-Carlo Rota on combinatorics", Contemp. Mathematicians, 1995, Birkhuser Boston, Boston, MA, 504-512.

[65] W. Rühl and A. V. Turbiner, Exact solvability of the Calogero and Sutherland models, Mod. Phys. Lett., 1995, A10, 2213-2222.

[66] S. Sawada and T. Kotera, A method for findind $N$-soliton solutions of the KdV and KdV-like equation, Prog. Theor. Phys., 1974, 51, $1355-1367$.

[67] T. Schedler, Trigonometric solutions of the associative YangBaxter equation, Math. Res. Lett., 2003, 10(2-3), 301-321.

[68] M. A. Semenov-Tian-Shansky, What is a classical rr-matrix?, Funct. Anal. Appl., 1983, 17(4), 259-272.

[69] E. K. Sklyanin, On the complete integrability of the Landau-Lifshitz equation, Preprint No. E-3, Leningrad Branch Math. Inst. (LOMI), 1979, Leningrad. (in Russian)

[70] T. Skrypnyk, Deformations of loop algebras and classical integrable systems: Finite-dimensional Hamiltonian systems, Reviews in Math. Phys., 2004, 16(7), 823-849.

[71] V. V. Sokolov, A new integrable case for the Kirchhoff equation, Theor. Math. Phys., 2001, 129(1), 1335-1340.

[72] V. V. Sokolov, Algebraic quantum Hamiltonians on the plane, Theor. Math. Phys., 2015, 184(1), 940-952.

[73] V. V. Sokolov, Classification of constant solutions for associative Yang-Baxter equation on $\mathrm{Mat}_{3}$, Theor. Math. Phys., 2013, 176(3), 385-392.

[74] V. V. Sokolov, Elliptic Calogero-Moser Hamiltonians and compatible Poisson brackets, in Recent developments in Integrable Systems and related topics of Mathematical Physics, Springer series PROMS, editors V. M. Buchstaber, S. Konstantinou-Rizos and A. V. Mikhailov, 2018, arXiv 1608.08511 in hep-th.

[75] V. V. Sokolov, Hamiltonian property of the Krichever-Novikov equation, Sov. Math. Dokl., 1984, 30, 44-47. 
[76] V. V. Sokolov, On decompositions of the loop algebra over $\mathfrak{s o}_{3}$ into a sum of two subalgebras, Doklady Mathematics, 2004, 70(1), 568570 .

[77] V. V. Sokolov and A. B. Shabat, $(L-A)$-pairs and Ricatti-type substitutions, Funct. Anal. Appl., 1980, 14(2), 148-150.

[78] V. V. Sokolov V. V. and S. I. Svinolupov, Deformation of nonassociative algebras and integrable differential equations, Acta Applicandae Mathematica, 1995, 41(1-2), 323-339.

[79] V. V. Sokolov V. V. and A. V. Tsyganov, On Lax pairs for the generalized Kowalewski and Goryachev-Chaplygin tops, Theor. Math. Phys., 2002, 131(1), 543-549.

[80] V. V. Sokolov and A. V. Turbiner, Quasi-exact-solvability of the $A_{2}$ Elliptic model: algebraic form, $\mathfrak{s l}(3)$ hidden algebra, polynomial eigenfunctions, Journal Physics A, 2015, 48(15), 155201.

[81] K. Takemura, Quasi-exact solvability of Inozemtsev models J. Phys. A: Math. Gen., 2002, 35, 8867-8881.

[82] M. Van den Bergh, Double Poisson algebras, Trans. Amer. Math. Soc., 2008, 360(11), 555-603.

[83] A. P. Veselov, On Darboux-Treibich-Verdier potentials, Lett. Math. Phys. 2011, 96(1-3), 209-216.

[84] E. B. Vinberg, Convex homogeneous cones, Transl. Moscou Math. Soc., 1963, 12, 340-403.

[85] E. B. Vinberg, Disete linear groups that are generated by reflections, Izv. Akad. Nauk SSSR, Ser. Mat., 1971, 35, 1072-1112.

[86] E. B. Vinberg, On certain commutative subalgebras of a universal enveloping algebra, Izvestia AN SSSR, 1991, 36(1), 1-22.

[87] V. E. Zakharov and A. V. Mikhailov, Method of the inverse scattering problem with spectral parameter on an algebraic curve, Functional Analysis and Its Applications, 1983, 17(4), 247-251.

[88] V. E. Zakharov and A. B. Shabat, Exact theory of two-dimensional self-focusing and one-dimensional self-modulation of waves in nonlinear media, Soviet Physics JETP, 1972, 34(1), 62-69.

[89] V. E. Zakharov and A. B. Shabat, Integration of nonlinear equations of mathematical physics by the method of inverse scattering. II, Funct. Anal. Appl., 1979, 13(3), 166-174. 
[90] A. I. Zobnin, Anti-Frobenius algebras and associative Yang-Baxter equation, Matem. Mod., 2014, 26(11), 51-56. (in Russian)

Vladimir Sokolov

Landau Institute for Theoretical Physics

142432 Chernogolovka (Moscow region), Russia vsokolov@landau.ac.ru

Instituto de Matemática e Estatística, Universidade de São Paulo 05508-090, São Paulo, Brasil

sokolov@ime.usp.br

http://www.ime.usp.br 